\title{
Étude d'une somme arithmétique multiple liée à la fonction de Möbius
}

\author{
par \\ Michel Balazard (Marseille), Mongi Naimi (Tunis) \\ et Y.-F. S. PÉtermann (Genève) (première partie) \\ et \\ Mongi Naimi (Tunis) et Y.-F. S. PÉtermann (Genève) (deuxième partie)
}

\section{Table des matières}

0 . Introduction. La somme arithmétique $S_{r}$

1. Première partie. Une formule de Perron effective en $r$ variables 247

$\begin{array}{ll}\text { 1.1. Notations et remarques préliminaires } & 247\end{array}$

1.2. Le semi-groupe multiplicatif $\mathbb{N}^{* r}, r \geq 2 \quad 249$

1.3. Fonctions arithmétiques de $r$ variables $\quad 249$

1.4. Séries de Dirichlet en $r$ variables 250

1.5. Formules de Perron en $r$ variables 250

1.6. Application à la somme $S_{r} \quad 253$

1.6.1. Définition 253

1.6.2. Abscisses de convergence absolue 253

1.6.3. Les fractions rationnelles $\mathcal{Q}_{r}\left(X_{1}, \ldots, X_{r} ; T\right)$ et $Q_{r}\left(X_{1}, \ldots, X_{r} ; T\right) \quad 254$

1.6.4. Les séries de Dirichlet $\widetilde{F}\left(s_{1}, \ldots, s_{r}\right)$ et $F\left(s_{1}, \ldots, s_{r}\right) \quad 256$

1.6.5. Estimation de l'erreur de troncature 259

2. Deuxième partie. Démonstration du Théorème 1

2.1. Remerciements 264

2.2. Notations 264

2.3. Structure de la démonstration 265

2.3.1. Plan 265

2.3.2. Le mécanisme de récurrence : description générale 265

$\begin{array}{ll}\text { 2.3.3. Pôles et résidus } & 268\end{array}$

2.4. Suites de pôles $\quad 271$

$\begin{array}{ll}\text { 2.4.1. Quelques résultats auxiliaires } & 272\end{array}$

2.5. Les facteurs sans pôle des résidus successifs 273

2.6. Les trois premiers pas du procédé d'évaluation 276

2000 Mathematics Subject Classification: Primary 11N64; Secondary 11A25, 11M06, $15 \mathrm{~A} 04,32 \mathrm{~S} 45$.

Key words and phrases: Möbius function, distribution function, Hamburger moment, prime number theorem, Perron's inversion formula, Riemann zeta function, linear transformation, several complex variables, singularities. 
2.7. Le $m$-ème pas 282

2.7.1. Conclusion de l'argument : preuves de (50), (55) et (56) 286

2.8. Autres résultats auxiliaires 288

2.9. Choix des abscisses d'intégration $\quad 289$

2.10. Démonstration du Théorème $1 \quad 291$

$\begin{array}{ll}\text { Références } & 297\end{array}$

\section{INTRODUCTION \\ LA SOMME ARITHMÉTIQUE $S_{r}$}

Soit $\mu$ la fonction de Möbius et $x$ un (grand) nombre réel positif. Ce travail, comme déjà [DIT], a pour objet l'étude du moment d'ordre $r$,

de la fonction arithmétique

$$
S_{r}(x)=\lim _{y \rightarrow \infty} y^{-1} \sum_{n \leq y} M(n, x)^{r},
$$

$$
M(n, x):=\sum_{d \mid n, d \leq x} \mu(d)
$$

(qui est bien sûr nulle dès que $x \geq n>1$ ). Ce moment peut s'écrire sous la forme

$$
S_{r}(x)=\sum_{\substack{n_{j} \leq x \\ j=1, \ldots, r}} \frac{\mu\left(n_{1}\right) \cdots \mu\left(n_{r}\right)}{\left[n_{1}, \ldots, n_{r}\right]} .
$$

Le propos de F. Dress, H. Iwaniec et G. Tenenbaum [DIT] est d'établir la convergence de $S_{2}(x)$ vers une limite strictement positive, pour laquelle ils donnent en outre trois expressions explicites. Y. Motohashi [M1, M2] montre que $S_{3}(x)=o(1)$ et que, pour des constantes $c_{i}$, on a $S_{4}(x)=$ $c_{2} \log ^{2} x+c_{1} \log x+c_{0}+o(1)$, puis propose au lecteur de considérer le cas général.

C'est ce que nous faisons dans ce travail.

Nous notons génériquement $\delta(x)$ toute fonction

$$
\delta_{C}(x):=\exp \left(-C(\log x)^{3 / 5}(\log \log x)^{-1 / 5}\right),
$$

où $C$ est une constante réelle positive. (Nous pouvons ainsi écrire par exemple $\delta(x)^{2}=\delta(x)$ et, quelle que soit $C, \delta(x) \exp \left(C(\log x)^{2 / 5}\right) \leq \delta(x)$.)

ThÉORÈMe 1. (i) Pour chaque entier positif $r$ il existe un polynôme $P_{r}$ tel que

$$
S_{r}(x)-P_{r}(\log x) \ll \delta(x) \quad(x \rightarrow \infty) .
$$

(ii) Si de plus $r$ est impair le polynôme $P_{r}$ est identiquement nul, i.e.

$$
S_{r}(x) \ll \delta(x) \quad(x \rightarrow \infty) .
$$

Remarque 1. Pour $r=1$ le (ii) du théorème est une forme équivalente bien connue du théorème des nombres premiers. 
La démonstration complète est longue, et nous la présentons en deux parties. Dans la première partie nous transformons, à un terme d'erreur près, une somme arithmétique multiple d'une fonction $f\left(n_{1}, \ldots, n_{r}\right)$ multiplicative en une intégrale multiple tronquée, obtenant ainsi ce qu'il est naturel d'appeler une "formule de Perron effective en $r$ variables". Dans ce but nous nous appuyons sur le livre de G. Tenenbaum [T], qui traite du cas $r=1$. Nous montrons alors que cette formule générale est applicable à la somme arithmétique multiple $S_{r}(x)$, l'erreur commise n'étant pas plus grande qu'un $\delta(x)$ : voir le Théorème 2 au 1.6.5.

Dans la deuxième partie nous évaluons l'intégrale multiple tronquée ainsi obtenue, ce qui livre finalement le théorème.

Remarque 2. Dans un travail récent, $R$. de la Bretèche $[B]$ développe une méthode assez générale permettant de traiter des sommes multiples très similaires à $S_{k}(x)$. Ses résultats ne s'appliquent cependant pas à $S_{k}(x)$ si l'on n'assume pas que la fonction génératrice associée à $f\left(n_{1}, \ldots, n_{r}\right)=$ $\mu\left(n_{1}\right) \cdots \mu\left(n_{r}\right) /\left[n_{1}, \ldots, n_{r}\right]$ satisfait une certaine hypothèse non démontrée, équivalente à l'hypothèse de Riemann, ou à une hypothèse légèrement plus faible concernant les zéros de la fonction $\zeta$ de Riemann. On peut obtenir le résultat suivant avec $[\mathrm{B}]$, après s'être assuré que l'hypothèse (i) du Théorème 2 de ce travail est bien satisfaite.

THÉORÈmE 1HR. Si l'hypothèse de Riemann est satisfaite, il existe des polynômes $P_{r}$ et des nombres positifs $\vartheta_{r}(r \geq 1)$ satisfaisant:

(i) Pour chaque entier positif $r$ on a

$$
S_{r}(x)-P_{r}(\log x) \ll x^{-\vartheta_{r}} \quad(x \rightarrow \infty) .
$$

(ii) Si de plus $r$ est impair le polynôme $P_{r}$ est identiquement nul, i.e.

$$
S_{r}(x) \ll x^{-\vartheta_{r}} \quad(x \rightarrow \infty) .
$$

\section{PREMIÈrE PARTIE}

UNE FORMULE DE PERRON EFFECTIVE EN $r$ VARIABLES

\subsection{Notations et remarques préliminaires}

- On pose

$$
h(y)= \begin{cases}0 & \text { si } 0<y<1 \\ 1 / 2 & \text { si } y=1 \\ 1 & \text { si } y>1\end{cases}
$$

Pour $y>0$ et $\sigma>0$, on a $h(y)+h(1 / y)=1$ et $h(y) \leq y^{\sigma}$.

- L'apostrophe dans

$$
\sum_{n_{1} \leq x_{1}, \ldots, n_{r} \leq x_{r}}^{\prime} f\left(n_{1}, \ldots, n_{r}\right)
$$


indique une somme normalisée, c'est-à-dire valant

$$
\sum_{n_{1} \leq x_{1}, \ldots, n_{r} \leq x_{r}} f\left(n_{1}, \ldots, n_{r}\right) h\left(\frac{x_{1}}{n_{1}}\right) \cdots h\left(\frac{x_{r}}{n_{r}}\right) .
$$

- Pour $y>0$, on pose $y^{*}=\max (1, y)$. On a donc

$$
\frac{1}{y^{*}}=\int_{y}^{\infty} h(t) \frac{d t}{t^{2}}
$$

- Pour $y, \kappa, T$ positifs, posons

$$
\Delta(y, \kappa, T):=\frac{1}{2 \pi i} \int_{\kappa-i T}^{\kappa+i T} y^{s} \frac{d s}{s}-h(y) .
$$

- Posons, pour $x_{1}, \kappa_{1}, T_{1}, x_{2}, \kappa_{2}, T_{2}, \ldots, x_{r}, \kappa_{r}, T_{r}$ tous positifs,

$$
\begin{aligned}
& \Delta\left(x_{1}, \kappa_{1}, T_{1} ; \ldots ; x_{r}, \kappa_{r}, T_{r}\right) \\
& \quad:=\frac{1}{(2 \pi i)^{r}} \int_{\kappa_{r}-i T_{r}}^{\kappa_{r}+i T_{r}} \cdots \int_{\kappa_{1}-i T_{1}}^{\kappa_{1}+i T_{1}} x_{1}^{s_{1}} \cdots x_{r}^{s_{r}} \frac{d s_{1} \cdots d s_{r}}{s_{1} \cdots s_{r}}-h\left(x_{1}\right) \cdots h\left(x_{r}\right) .
\end{aligned}
$$

- Si $X_{1}, \ldots, X_{r}$ sont des indéterminées, on définit pour $E \subset\{1, \ldots, r\}$ les produits

$$
\Pi_{E}:=\prod_{i \in E} X_{i}
$$

Les polynômes symétriques élémentaires $\boldsymbol{\sigma}_{k}, 1 \leq k \leq r$, sont alors définis par

$$
\boldsymbol{\sigma}_{k}\left(X_{1}, \ldots, X_{r}\right):=\sum_{|E|=k} \Pi_{E},
$$

où $|E|$ désigne le nombre d'éléments de $E$.

- On pose

$$
\begin{aligned}
& P_{r}\left(X_{1}, \ldots, X_{r}\right)=\left(1+X_{1}\right) \cdots\left(1+X_{r}\right)-1=\sum_{k=1}^{r} \boldsymbol{\sigma}_{k}\left(X_{1}, \ldots, X_{r}\right), \\
& P_{r}^{*}\left(X_{1}, \ldots, X_{r}\right)=P_{r}\left(-X_{1}, \ldots,-X_{r}\right) .
\end{aligned}
$$

- Si $s_{1}, \ldots, s_{r}$ sont des nombres complexes, on pose, pour $E \subset\{1, \ldots, r\}$,

$$
S_{E}:=\sum_{i \in E} s_{i}
$$

On pose aussi

$$
\sigma_{i}:=\Re s_{i}, \quad t_{i}:=\Im s_{i} .
$$

- Le plus grand diviseur commun aux nombres entiers positifs $n_{1}, \ldots, n_{r}$ sera noté $\left\langle n_{1}, \ldots, n_{r}\right\rangle$; leur plus petit multiple commun sera noté $\left[n_{1}, \ldots, n_{r}\right]$. 
1.2. Le semi-groupe multiplicatif $\mathbb{N}^{* r}, r \geq 2$. Soit $r$ un nombre entier supérieur ou égal à 2 . On munit l'ensemble $\mathbb{N}^{* r}$ des $r$-uples de nombres entiers positifs d'une structure de semi-groupe multiplicatif en définissant le produit coordonnée par coordonnée :

$$
\left(n_{1}, \ldots, n_{r}\right) \cdot\left(m_{1}, \ldots, m_{r}\right):=\left(n_{1} m_{1}, \ldots, n_{r} m_{r}\right) .
$$

L'élément neutre est $(1, \ldots, 1)$. On a les notions usuelles de divisibilité et d'irréductibilité. Les éléments irréductibles sont les $\left(n_{1}, \ldots, n_{r}\right)$ où tous les $n_{i}$ valent 1 sauf l'un d'entre eux, qui est un nombre premier. Ils se groupent donc par paquets de $r$, correspondants à chaque nombre premier. Les éléments analogues aux puissances de nombres premiers sont les $\left(p^{\alpha_{1}}, \ldots, p^{\alpha_{r}}\right)$, où $p$ est premier et les $\alpha_{i}$ entiers naturels. Tout élément de $\mathbb{N}^{* r}$ a une décomposition unique en produit d'éléments de ce type :

$$
\left(n_{1}, \ldots, n_{r}\right)=\prod_{p}\left(p^{v_{p}\left(n_{1}\right)}, \ldots, p^{v_{p}\left(n_{r}\right)}\right),
$$

où $v_{p}$ désigne la valuation $p$-adique.

1.3. Fonctions arithmétiques de $r$ variables. Une fonction arithmétique de $r$ variables est une application $f: \mathbb{N}^{* r} \rightarrow \mathbb{C}$. Une telle fonction est dite multiplicative si $f(1, \ldots, 1)=1$ et

$$
f\left(n_{1} n_{1}^{\prime}, \ldots, n_{r} n_{r}^{\prime}\right)=f\left(n_{1}, \ldots, n_{r}\right) f\left(n_{1}^{\prime}, \ldots, n_{r}^{\prime}\right)
$$

à chaque fois que $n_{1} \cdots n_{r}$ et $n_{1}^{\prime} \cdots n_{r}^{\prime}$ sont premiers entre eux. (On prendra garde au fait que si l'égalité $\left\langle n_{1} \cdots n_{r}, n_{1}^{\prime} \cdots n_{r}^{\prime}\right\rangle=1$ entraîne bien que les $r$ uples $\left(n_{1}, \ldots, n_{r}\right)$ et $\left(n_{1}^{\prime}, \ldots, n_{r}^{\prime}\right)$ ont comme seul diviseur commun $(1, \ldots, 1)$, la réciproque est fausse.) Cette dernière condition équivaut à l'égalité

$$
f\left(p_{1}^{\alpha_{1,1}} \cdots p_{l}^{\alpha_{1, l}}, \ldots, p_{1}^{\alpha_{r, 1}} \cdots p_{l}^{\alpha_{r, l}}\right)=f\left(p_{1}^{\alpha_{1,1}}, \ldots, p_{1}^{\alpha_{r, 1}}\right) \cdots f\left(p_{l}^{\alpha_{1, l}}, \ldots, p_{l}^{\alpha_{r, l}}\right)
$$

pour tous nombres premiers deux à deux distincts $p_{1}, \ldots, p_{l}$ et tous nombres entiers $\alpha_{i, j}, 1 \leq i \leq r, 1 \leq j \leq l$, positifs ou nuls.

Deux exemples importants de fonctions multiplicatives sont fournis par le plus grand commun diviseur $\left(n_{1}, \ldots, n_{r}\right) \mapsto\left\langle n_{1}, \ldots, n_{r}\right\rangle$ et le plus petit commun multiple $\left(n_{1}, \ldots, n_{r}\right) \mapsto\left[n_{1}, \ldots, n_{r}\right]$.

On a un théorème du produit eulérien en $r$ variables.

Proposition 1. Si $f: \mathbb{N}^{* r} \rightarrow[0,+\infty[$ est multiplicative, la série et le produit infini

$$
\sum_{n_{1}, \ldots, n_{r}} f\left(n_{1}, \ldots, n_{r}\right) \quad \text { et } \prod_{p} \sum_{j_{1} \geq 0, \ldots, j_{r} \geq 0} f\left(p^{j_{1}}, \ldots, p^{j_{r}}\right)
$$

convergent ou divergent simultanément, et ont la même valeur.

On peut aussi exprimer sous forme d'un produit la somme obtenue en fixant les valeurs de certaines variables. 
Proposition 2. Soit $k \in \mathbb{N}^{*}$ tel que $1 \leq k<r$ et $a_{1}, \ldots, a_{k} \in \mathbb{N}^{*}$. Si $f: \mathbb{N}^{* r} \rightarrow[0,+\infty[$ est multiplicative, la série et le produit infini

$$
\sum_{n_{k+1}, \ldots, n_{r}} f\left(a_{1}, \ldots, a_{k}, n_{k+1}, \ldots, n_{r}\right)
$$

et

$$
\prod_{p} \sum_{j_{k+1} \geq 0, \ldots, j_{r} \geq 0} f\left(p^{v_{p}\left(a_{1}\right)}, \ldots, p^{v_{p}\left(a_{k}\right)}, p^{j_{k+1}}, \ldots, p^{j_{r}}\right)
$$

convergent ou divergent simultanément, et ont la même valeur.

Pour plus d'informations concernant cette théorie, le lecteur consultera avec profit l'article [D].

1.4. Séries de Dirichlet en $r$ variables. Si $f: \mathbb{N}^{* r} \rightarrow \mathbb{C}$ est une fonction arithmétique, on lui associe une série de Dirichlet

$$
F\left(s_{1}, \ldots, s_{r}\right):=\sum_{n_{1}, \ldots, n_{r}} \frac{f\left(n_{1}, \ldots, n_{r}\right)}{n_{1}^{s_{1}} \cdots n_{r}^{s_{r}}} .
$$

On suppose dans toute la suite que $s_{1}, \ldots, s_{r}$ sont des variables complexes, et on note $\sigma_{i}=\Re s_{i}$ pour $1 \leq i \leq r$. Observons que les inégalités $\sigma_{i}^{\prime}>\sigma_{i}$ pour tout $i$ tel que $1 \leq i \leq r$ et la convergence de

$$
\widetilde{F}\left(\sigma_{1}, \ldots, \sigma_{r}\right):=\sum_{n_{1}, \ldots, n_{r}} \frac{\left|f\left(n_{1}, \ldots, n_{r}\right)\right|}{n_{1}^{\sigma_{1}} \cdots n_{r}^{\sigma_{r}}}
$$

entraînent celle de $\widetilde{F}\left(\sigma_{1}^{\prime}, \ldots, \sigma_{r}^{\prime}\right)$. Cela permet de définir la notion de $r$ uple d'abscisses de convergence absolue de $F\left(s_{1}, \ldots, s_{r}\right)$ : c'est un $r$-uple $\left(\sigma_{1}, \ldots, \sigma_{r}\right)$ tel que $\widetilde{F}\left(\sigma_{1}^{\prime}, \ldots, \sigma_{r}^{\prime}\right)$ converge si $\sigma_{i}^{\prime}>\sigma_{i}$ pour tout $i$ tel que $1 \leq i \leq r$, et diverge si $\sigma_{i}^{\prime}<\sigma_{i}$ pour tout $i$ tel que $1 \leq i \leq r$. Un tel $r$-uple n'est pas nécessairement unique.

1.5. Formules de Perron en $r$ variables. Nous utiliserons les majorations données dans le chapitre II.2, Formules de sommation, de [T]. On a notamment le lemme suivant.

Proposition 3. On a uniformément

$$
|\Delta(y, \kappa, T)| \leq y^{\kappa} /(\pi T|\log y|)^{*} .
$$

Pour $r$ variables, on en déduit le résultat suivant.

Proposition 4. On a uniformément

$$
\begin{aligned}
& \left|\Delta\left(x_{1}, \kappa_{1}, T_{1} ; \ldots ; x_{r}, \kappa_{r}, T_{r}\right)\right| \\
& \quad \leq x_{1}^{\kappa_{1}} \cdots x_{r}^{\kappa_{r}} P_{r}\left(\frac{1}{\left(\pi T_{1}\left|\log x_{1}\right|\right)^{*}}, \ldots, \frac{1}{\left(\pi T_{r}\left|\log x_{r}\right|\right)^{*}}\right) .
\end{aligned}
$$


Preuve. Nous avons

$$
\frac{1}{(2 \pi i)^{r}} \int_{\kappa_{r}-i T_{r}}^{\kappa_{r}+i T_{r}} \cdots \int_{\kappa_{1}-i T_{1}}^{\kappa_{1}+i T_{1}} x_{1}^{s_{1}} \cdots x_{r}^{s_{r}} \frac{d s_{1} \cdots d s_{r}}{s_{1} \cdots s_{r}}=\prod_{j=1}^{r}\left(h\left(x_{j}\right)+\Delta\left(x_{j}, \kappa_{j}, T_{j}\right)\right),
$$

donc

$$
\begin{aligned}
\left|\Delta\left(x_{1}, \kappa_{1}, T_{1} ; \ldots ; x_{r}, \kappa_{r}, T_{r}\right)\right| & \\
& =\left|\prod_{j=1}^{r}\left(h\left(x_{j}\right)+\Delta\left(x_{j}, \kappa_{j}, T_{j}\right)\right)-h\left(x_{1}\right) \cdots h\left(x_{r}\right)\right| \\
& =\left|\sum_{J \subset\{1, \ldots, r\}} \prod_{j \notin J} h\left(x_{j}\right) \prod_{j \in J} \Delta\left(x_{j}, \kappa_{j}, T_{j}\right)\right| \\
& \leq \sum_{J \subset\{1, \ldots, r\}} \prod_{j \neq J} x_{j}^{\kappa_{j}} \prod_{j \in J} x_{j}^{\kappa_{j}} /\left(\pi T_{j}\left|\log x_{j}\right|\right)^{*} \\
& =x_{1}^{\kappa_{1} \ldots x_{r}^{\kappa_{r}}} \sum_{\substack{J \subset\{1, \ldots, r\} \\
J \neq \emptyset}} \prod_{j \in J} 1 /\left(\pi T_{j}\left|\log x_{j}\right|\right)^{*},
\end{aligned}
$$

d'où le résultat.

Par sommation, on déduit de la Proposition 4 la suivante.

Proposition 5 (Première formule de Perron effective). Soit $f: \mathbb{N}^{* r} \rightarrow$ $\mathbb{C}$ une fonction arithmétique de $r$ variables et $\left(\sigma_{a_{1}}, \ldots, \sigma_{a_{r}}\right)$ un r-uple d'abscisses de convergence absolue de la série de Dirichlet associée $F\left(s_{1}, \ldots, s_{r}\right)$. On a, pour $x_{1} \geq 1, \ldots, x_{r} \geq 1, \kappa_{1}>\max \left(0, \sigma_{a_{1}}\right), \ldots, \kappa_{r}>\max \left(0, \sigma_{a_{r}}\right)$, $T_{1} \geq 1, \ldots, T_{r} \geq 1$

$$
\begin{aligned}
& \mid \sum_{n_{1} \leq x_{1}, \ldots, n_{r} \leq x_{r}}^{\prime} f\left(n_{1}, \ldots, n_{r}\right) \\
& \quad-\frac{1}{(2 \pi i)^{r}} \int_{\kappa_{r}-i T_{r}}^{\kappa_{r}+i T_{r}} \ldots \int_{\kappa_{1}-i T_{1}}^{\kappa_{1}+i T_{1}} F\left(s_{1}, \ldots, s_{r}\right) x_{1}^{s_{1}} \cdots x_{r}^{s_{r}} \frac{d s_{1} \cdots d s_{r}}{s_{1} \cdots s_{r}} \mid \\
& \leq x_{1}^{\kappa_{1}} \ldots x_{r}^{\kappa_{r}} \sum_{n_{1} \geq 1, \ldots, n_{r} \geq 1} \frac{\left|f\left(n_{1}, \ldots, n_{r}\right)\right|}{n_{1}^{\kappa_{1}} \cdots n_{r}^{\kappa_{r}}} \\
& \quad \times P_{r}\left(\frac{1}{\left(\pi T_{1}\left|\log \left(x_{1} / n_{1}\right)\right|\right)^{*}}, \ldots, \frac{1}{\left(\pi T_{r}\left|\log \left(x_{r} / n_{r}\right)\right|\right)^{*}}\right) .
\end{aligned}
$$

Donnons-nous un corollaire spécifique, qui sera utile plus loin, au cas d'une fonction $f$ symétrique, c'est-à-dire telle que $f\left(n_{1}, \ldots, n_{r}\right)=f\left(n_{\alpha 1}\right.$, $\ldots, n_{\alpha r}$ ) pour $n_{1} \geq 1, \ldots, n_{r} \geq 1$ et $\alpha \in \mathfrak{S}_{r}$ (où $\mathfrak{S}_{r}$ désigne le groupe des permutations de $r$ objets). Nous supposons de plus que les bornes 
d'intégration $\kappa_{j} \pm i T_{j}$ sont de la forme $N_{j} \kappa \pm i M_{j} T$, avec $1=N_{1} \leq \cdots \leq N_{r}$ et $1=M_{1} \leq \cdots \leq M_{r}$.

Posons au préalable pour $1 \leq k \leq r$,

$$
F_{r}^{\mathcal{Q}}(\sigma, x, T):=\sum_{n_{1}, \ldots, n_{r}} \frac{\left|f\left(n_{1}, \ldots, n_{r}\right)\right|\left(n_{1} \cdots n_{r}\right)^{-\sigma}}{\min _{j=1, \ldots, r}\left(T\left|\log \left(x / n_{j}\right)\right|+1\right)} .
$$

Proposition 6 (Deuxième formule de Perron effective). Soit $f: \mathbb{N}^{* r}$ $\rightarrow \mathbb{C}$ une fonction arithmétique symétrique de $r$ variables et $\left(\sigma_{a}, \ldots, \sigma_{a}\right)$ un $r$-uple diagonal d'abscisses de convergence absolue de la série de Dirichlet associée $F\left(s_{1}, \ldots, s_{r}\right)$. On a, pour $x \geq 2, T \geq 2, \sigma \leq \sigma_{a}, \delta>0, \kappa=\sigma_{a}-$ $\sigma+\delta / \log x, 1=N_{1} \leq \cdots \leq N_{r}, 1=M_{1} \leq \cdots \leq M_{r}$ et $N_{0}:=N_{1}+\cdots+N_{r}$,

$$
\begin{aligned}
& \mid \sum_{n_{j} \leq x}^{\prime} \frac{f\left(n_{1}, \ldots, n_{r}\right)}{\left(n_{1} \cdots n_{r}\right)^{s}}-\frac{1}{(2 \pi i)^{r}} \int_{N_{1} \kappa-i M_{1} T}^{N_{1} \kappa+i M_{1} T} \cdots \\
& \quad \ldots \int^{N_{r} \kappa+i M_{r} T} F\left(s+w_{1}, \ldots, s+w_{r}\right) x^{w_{1}+\cdots+w_{r}} \frac{d w_{1} \cdots d w_{r}}{w_{1} \cdots w_{r}} \mid \\
& \quad \ll x^{N_{0} \kappa-i M_{r} T} F^{\left.\sigma_{a}-\sigma\right)} F_{r}^{\bigcirc}\left(\sigma_{a}+\delta / \log x, x, T\right) .
\end{aligned}
$$

Preuve. On applique la première formule de Perron effective à la fonction $g\left(n_{1}, \ldots, n_{r}\right):=f\left(n_{1}, \ldots, n_{r}\right) /\left(n_{1} \cdots n_{r}\right)^{s}$. La série de Dirichlet associée est $G\left(w_{1}, \ldots, w_{r}\right):=F\left(s+w_{1}, \ldots, s+w_{r}\right) ;\left(\sigma_{a}-\sigma, \ldots, \sigma_{a}-\sigma\right)$ en est un $r$-uple d'abscisses de convergence absolue. Pour $x \geq 2, T \geq 2, \sigma \leq \sigma_{a}, \delta>0$ et $\kappa=\sigma_{a}-\sigma+\delta / \log x$, le module à majorer est donc

$$
\begin{aligned}
& \leq x^{N_{0} \kappa} \sum_{n_{1}, \ldots, n_{r}} \frac{\left|f\left(n_{1}, \ldots, n_{r}\right)\right|}{n_{1}^{\sigma+N_{1} \kappa} \cdots n_{r}^{\sigma+N_{r} \kappa}} P_{r}\left(\frac{1}{\left(\pi M_{1} T\left|\log \frac{x}{n_{1}}\right|\right)^{*}}, \ldots, \frac{1}{\left(\pi M_{r} T\left|\log \frac{x}{n_{r}}\right|\right)^{*}}\right) \\
& \leq x^{N_{0} \kappa} \sum_{n_{1}, \ldots, n_{r}} \frac{\left|f\left(n_{1}, \ldots, n_{r}\right)\right|}{\left(n_{1} \cdots n_{r}\right)^{\sigma+\kappa}} \sum_{k=1}^{r} \sigma_{k}\left(\frac{1}{\left(\pi T\left|\log \frac{x}{n_{1}}\right|\right)^{*}}, \ldots, \frac{1}{\left(\pi T\left|\log \frac{x}{n_{r}}\right|\right)^{*}}\right) \\
& =x^{N_{0} \kappa} \sum_{k=1}^{r} \sum_{n_{1}, \ldots, n_{r}} \frac{\left|f\left(n_{1}, \ldots, n_{r}\right)\right|}{\left(n_{1} \cdots n_{r}\right)^{\sigma+\kappa}} \sum_{1 \leq i_{1}<\cdots<i_{k} \leq r} \frac{1}{\left(\pi T\left|\log \frac{x}{n_{i_{1}}}\right|\right)^{*} \cdots\left(\pi T\left|\log \frac{x}{n_{i_{k}}}\right|\right)^{*}} \\
& =x^{N_{0} \kappa} \sum_{k=1}^{r} \sum_{1 \leq i_{1}<\cdots<i_{k} \leq r} \sum_{n_{1}, \ldots, n_{r}} \frac{\left|f\left(n_{1}, \ldots, n_{r}\right)\right| /\left(n_{1} \cdots n_{r}\right)^{\sigma+\kappa}}{\left(\pi T\left|\log \frac{x}{n_{i_{1}}}\right|\right)^{*} \cdots\left(\pi T\left|\log \frac{x}{n_{i_{k}}}\right|\right)^{*} .}
\end{aligned}
$$

Comme $f$ est symétrique, la somme intérieure est indépendante de $\left(i_{1}, \ldots, i_{k}\right)$ et le majorant obtenu est donc 


$$
\begin{array}{r}
x^{N_{0} \kappa} \sum_{k=1}^{r}\left(\begin{array}{l}
r \\
k
\end{array}\right) \sum_{n_{1}, \ldots, n_{r}} \frac{\left|f\left(n_{1}, \ldots, n_{r}\right)\right| /\left(n_{1} \cdots n_{r}\right)^{\sigma+\kappa}}{\left(\pi T\left|\log \left(x / n_{1}\right)\right|\right)^{*} \cdots\left(\pi T\left|\log \left(x / n_{k}\right)\right|\right)^{*}} \\
\ll x^{N_{0}\left(\sigma_{a}-\sigma\right)} F_{r}^{\odot}\left(\sigma_{a}+\delta / \log x, x, T\right) .
\end{array}
$$

\subsection{Application à la somme $S_{r}$}

1.6.1. Définition. On pose

$$
f\left(n_{1}, \ldots, n_{r}\right)=\frac{\mu\left(n_{1}\right) \cdots \mu\left(n_{r}\right)}{\left[n_{1}, \ldots, n_{r}\right]} .
$$

La fonction $f$ est donc symétrique et multiplicative. On a, pour $\left(j_{1}, \ldots, j_{r}\right) \in$ $\mathbb{N}^{r}$ et $p$ premier,

$$
f\left(p^{j_{1}}, \ldots, p^{j_{r}}\right)= \begin{cases}1 & \text { si } j_{1}=\cdots=j_{r}=0, \\ (-1)^{j_{1}+\cdots+j_{r}} / p & \text { si } \max \left(j_{1}, \ldots, j_{r}\right)=1, \\ 0 & \text { si } \max \left(j_{1}, \ldots, j_{r}\right) \geq 2 .\end{cases}
$$

1.6.2. Abscisses de convergence absolue. On en déduit, pour $s_{1}, \ldots, s_{r}$ complexes et $p$ premier,

$$
\begin{aligned}
\sum_{j_{1} \geq 0, \ldots, j_{r} \geq 0} \frac{f\left(p^{j_{1}}, \ldots, p^{j_{r}}\right)}{\left(p^{j_{1}}\right)^{s_{1}} \cdots\left(p^{j_{r}}\right)^{s_{r}}} & =1+\sum_{k=1}^{r} \frac{1}{p} \boldsymbol{\sigma}_{k}\left(-p^{-s_{1}}, \ldots,-p^{-s_{r}}\right) \\
& =1+\frac{1}{p} P_{r}^{*}\left(p^{-s_{1}}, \ldots, p^{-s_{r}}\right)
\end{aligned}
$$

et

$$
\begin{aligned}
\sum_{j_{1} \geq 0, \ldots, j_{r} \geq 0} \frac{\left|f\left(p^{j_{1}}, \ldots, p^{j_{r}}\right)\right|}{\left(p^{j_{1}}\right)^{s_{1}} \cdots\left(p^{j_{r}}\right)^{s_{r}}} & =1+\sum_{k=1}^{r} \frac{1}{p} \boldsymbol{\sigma}_{k}\left(p^{-s_{1}}, \ldots, p^{-s_{r}}\right) \\
& =1+\frac{1}{p} P_{r}\left(p^{-s_{1}}, \ldots, p^{-s_{r}}\right) .
\end{aligned}
$$

Pour $x_{1} \geq 0, \ldots, x_{r} \geq 0$, on a $P_{r}\left(x_{1}, \ldots, x_{r}\right) \geq x_{1}+\cdots+x_{r}$. Par conséquent, si $\sigma_{i} \leq 0$ pour au moins un $i \in\{1, \ldots, r\}$, on a pour, tout $p$,

$$
\sum_{j_{1} \geq 0, \ldots, j_{r} \geq 0} \frac{\left|f\left(p^{j_{1}}, \ldots, p^{j_{r}}\right)\right|}{\left(p^{j_{1}}\right)^{\sigma_{1}} \cdots\left(p^{j_{r}}\right)^{\sigma_{r}}} \geq 1+\frac{1}{p},
$$

donc la série $\widetilde{F}\left(\sigma_{1}, \ldots, \sigma_{r}\right)$ définie par (3) diverge, d'après la Proposition 1.

D'autre part, toujours pour $x_{1} \geq 0, \ldots, x_{r} \geq 0$, et en posant $x=$ $\max \left(x_{1}, \ldots, x_{r}\right)$, on a

$$
P_{r}\left(x_{1}, \ldots, x_{r}\right)=\left(1+x_{1}\right) \cdots\left(1+x_{r}\right)-1 \leq(1+x)^{r}-1 \leq 2^{r} x,
$$


pourvu que $x \leq 1$. Par conséquent,

$$
1 \leq \sum_{j_{1} \geq 0, \ldots, j_{r} \geq 0} \frac{\left|f\left(p^{j_{1}}, \ldots, p^{j_{r}}\right)\right|}{\left(p^{j_{1}}\right)^{\sigma_{1}} \cdots\left(p^{j_{r}}\right)^{\sigma_{r}}} \leq 1+\frac{2^{r}}{p^{1+\sigma}},
$$

où $\sigma=\min \left(\sigma_{1}, \ldots, \sigma_{r}\right)$. Cela prouve que la série $\widetilde{F}\left(\sigma_{1}, \ldots, \sigma_{r}\right)$ converge si tous les $\sigma_{i}$ sont positifs. Ainsi $(0, \ldots, 0)$ est un $r$-uple d'abscisses de convergence absolue de la série $F\left(s_{1}, \ldots, s_{r}\right)$ définie par (2).

1.6.3. Les fractions rationnelles $\mathcal{Q}_{r}\left(X_{1}, \ldots, X_{r} ; T\right)$ et $Q_{r}\left(X_{1}, \ldots, X_{r} ; T\right)$. Nous définissons les fractions rationnelles $\mathcal{Q}_{r}\left(X_{1}, \ldots, X_{r} ; T\right)$ et $Q_{r}\left(X_{1}, \ldots\right.$, $\left.X_{r} ; T\right)$ par les relations

$$
\begin{aligned}
1+T P_{r}\left(X_{1}, \ldots, X_{r}\right) & =1+T\left(\left(1+X_{1}\right) \cdots\left(1+X_{r}\right)-1\right) \\
& =: \frac{\mathcal{Q}_{r}\left(X_{1}, \ldots, X_{r} ; T\right)}{\prod_{E \neq \emptyset}\left(1-T \Pi_{E}\right)}
\end{aligned}
$$

où $E$ décrit l'ensemble des parties non vides de $\{1, \ldots, r\}$, et

$$
\begin{aligned}
1+T P_{r}^{*}\left(X_{1}, \ldots, X_{r}\right) & =1+T\left(\left(1-X_{1}\right) \cdots\left(1-X_{r}\right)-1\right) \\
& =: \frac{\prod_{|I| \text { impair }}\left(1-T \Pi_{I}\right)}{\prod_{|P| \text { pair }, P \neq \emptyset}\left(1-T \Pi_{P}\right)} Q_{r}\left(X_{1}, \ldots, X_{r} ; T\right),
\end{aligned}
$$

où $I$ et $P$ sont restreints aux éléments de l'ensemble des parties de $\{1, \ldots, r\}$.

Comme $\mathcal{Q}_{r}-1$ et $Q_{r}-1$ sont chacun sans terme constant, les séries formelles $\log \mathcal{Q}_{r}$ et $\log Q_{r}$ sont toutes deux bien définies.

Proposition 7. On a

$$
\log \mathcal{Q}_{r}=\sum_{n \geq 2} \mathcal{A}_{n} \frac{T^{n}}{n}
$$

où

$$
\mathcal{A}_{n}:=(-1)^{n-1} P_{r}\left(X_{1}, \ldots, X_{r}\right)^{n}-P_{r}\left(X_{1}^{n}, \ldots, X_{r}^{n}\right) \quad\left(\text { donc } \mathcal{A}_{1}=0\right) .
$$

Proposition 8. On a

$$
\log Q_{r}=\sum_{n \geq 2} A_{n} \frac{T^{n}}{n}
$$

où

$$
A_{n}:=(-1)^{n-1} P_{r}^{*}\left(X_{1}, \ldots, X_{r}\right)^{n}-P_{r}^{*}\left(X_{1}^{n}, \ldots, X_{r}^{n}\right) \quad\left(\text { donc } A_{1}=0\right) .
$$

Les preuves des Propositions 7 et 8 sont très similaires; nous nous contentons de démontrer cette dernière. 
Preuve de la Proposition 8. On a

$$
\begin{aligned}
\log Q_{r} & =\log \left(1+T P_{r}^{*}\right)+\sum_{|P| \text { pair }, P \neq \emptyset} \log \left(1-T \Pi_{P}\right)-\sum_{|I| \text { impair }} \log \left(1-T \Pi_{I}\right) \\
& =\sum_{n \geq 1}\left((-1)^{n-1}\left(P_{r}^{*}\right)^{n}-\sum_{|P| \text { pair }, P \neq \emptyset} \Pi_{P}^{n}+\sum_{|I| \text { impair }} \Pi_{I}^{n}\right) \frac{T^{n}}{n} .
\end{aligned}
$$

Le résultat en découle, puisque

$$
\sum_{|P| \text { pair }, P \neq \emptyset} \Pi_{P}^{n}-\sum_{|I| \text { impair }} \Pi_{I}^{n}=P_{r}^{*}\left(X_{1}^{n}, \ldots, X_{r}^{n}\right) .
$$

Donnons maintenant des majorations de valeurs prises par $\mathcal{Q}_{r}$ et $Q_{r}$.

Proposition 9. Soit $x_{1}, \ldots, x_{r}, t$ des nombres complexes, et $x$ et $u$ des nombres réels positifs tels que $|t| \leq u$ et $\left|x_{i}\right| \leq x$ pour $1 \leq i \leq r$.

(A) On a

$$
\left|\mathcal{Q}_{r}\left(x_{1}, \ldots, x_{r} ; t\right)\right| \leq\left(1-u+u(1+x)^{r}\right) \prod_{i=1}^{r}\left(1+u x^{i}\right)\left(\begin{array}{c}
r \\
i
\end{array}\right) .
$$

(B) $\operatorname{Si}(1+x)^{r}<1+1 / u$, on a

$$
\left|\log \mathcal{Q}_{r}\left(x_{1}, \ldots, x_{r} ; t\right)\right| \leq \frac{\left((1+x)^{r}-1\right)^{2} u^{2}}{1+u-u(1+x)^{r}} .
$$

Proposition 10. Soit $x_{1}, \ldots, x_{r}, t$ des nombres complexes, et $x$ et $u$ des nombres réels positifs tels que $|t| \leq u$ et $\left|x_{i}\right| \leq x$ pour $1 \leq i \leq r$.

(A) Si $x \geq 1$ et $u x^{r}<1$, on a

$$
\left|Q_{r}\left(x_{1}, \ldots, x_{r} ; t\right)\right| \leq\left(1-u+u(1+x)^{r}\right) \frac{\prod_{0<2 i \leq r}\left(1+u x^{2 i}\right)^{\left(\begin{array}{c}
r \\
2 i
\end{array}\right)}}{\prod_{0<2 j-1 \leq r}\left(1-u x^{2 j-1}\right)^{\left(\begin{array}{c}
r \\
2 j-1
\end{array}\right)}} .
$$

(B) $S i(1+x)^{r}<1+1 / u$, on $a$

$$
\left|\log Q_{r}\left(x_{1}, \ldots, x_{r} ; t\right)\right| \leq \frac{\left((1+x)^{r}-1\right)^{2} u^{2}}{1+u-u(1+x)^{r}} .
$$

Les preuves des Propositions 9 et 10 sont très similaires; nous nous contentons de démontrer cette dernière.

Preuve de la Proposition 10. La première inégalité résulte de la définition de $Q_{r}$.

Pour la deuxième, on majore d'abord $\left|A_{n}\left(x_{1}, \ldots, x_{r}\right)\right|$ :

$$
\begin{aligned}
\left|A_{n}\left(x_{1}, \ldots, x_{r}\right)\right| & =\left|(-1)^{n-1} P_{r}^{*}\left(x_{1}, \ldots, x_{r}\right)^{n}-P_{r}^{*}\left(x_{1}^{n}, \ldots, x_{r}^{n}\right)\right| \\
& \leq P_{r}(x, \ldots, x)^{n}+P_{r}\left(x^{n}, \ldots, x^{n}\right) \\
& =\left((1+x)^{r}-1\right)^{n}+\left(1+x^{n}\right)^{r}-1 .
\end{aligned}
$$


Or $\left(1+x^{n}\right)^{r}-1 \leq\left((1+x)^{r}-1\right)^{n}$, car ce sont deux polynômes en $x$ à coefficients positifs ou nuls; le coefficient de degré $j n$ du premier est $\left(\begin{array}{l}r \\ j\end{array}\right)$, alors que celui du second est au moins $\left(\begin{array}{l}r \\ j\end{array}\right)^{n}, 1 \leq j \leq r$. On a donc

$$
\left|A_{n}\left(x_{1}, \ldots, x_{r}\right)\right| \leq 2\left((1+x)^{r}-1\right)^{n},
$$

ce qui donne

$$
\left|\log Q_{r}\left(x_{1}, \ldots, x_{r} ; t\right)\right| \leq 2 \sum_{n \geq 2}\left((1+x)^{r}-1\right)^{n} \frac{u^{n}}{n} \leq \frac{\left((1+x)^{r}-1\right)^{2} u^{2}}{1-u\left((1+x)^{r}-1\right)},
$$

pourvu que $u\left((1+x)^{r}-1\right)<1$.

1.6.4. Les séries de Dirichlet $\widetilde{F}\left(s_{1}, \ldots, s_{r}\right)$ et $F\left(s_{1}, \ldots, s_{r}\right)$

Proposition 11. Pour $\sigma_{1}>0, \ldots, \sigma_{r}>0$, on $a$

$$
\widetilde{F}\left(s_{1}, \ldots, s_{r}\right)=\prod_{E \neq \emptyset} \zeta\left(1+S_{E}\right) \cdot \mathcal{G}\left(s_{1}, \ldots, s_{r}\right),
$$

où

$$
\mathcal{G}\left(s_{1}, \ldots, s_{r}\right):=\prod_{p} \mathcal{Q}_{r}\left(p^{-s_{1}}, \ldots, p^{-s_{r}} ; p^{-1}\right) .
$$

Pour tout $c$ tel que $0<c<1 / 2$, la fonction $\mathcal{G}\left(s_{1}, \ldots, s_{r}\right)$ se prolonge holomorphiquement au produit des demi-plans $\sigma_{i} \geq-c / r$ et y vérifie

$$
\left|\mathcal{G}\left(s_{1}, \ldots, s_{r}\right)\right| \leq \zeta(2-2 c)^{2^{2 r+2}-2^{r+1}} .
$$

Proposition 12. Pour $\sigma_{1}>0, \ldots, \sigma_{r}>0$, on a

$$
F\left(s_{1}, \ldots, s_{r}\right)=\frac{\prod_{P \neq \emptyset,|P| \text { pair }} \zeta\left(1+S_{P}\right)}{\prod_{|I| \text { impair }} \zeta\left(1+S_{I}\right)} \cdot H\left(s_{1}, \ldots, s_{r}\right),
$$

où

$$
H\left(s_{1}, \ldots, s_{r}\right):=\prod_{p} Q_{r}\left(p^{-s_{1}}, \ldots, p^{-s_{r}} ; p^{-1}\right) .
$$

Pour tout $c$ tel que $0<c<1 / 2$, la fonction $H\left(s_{1}, \ldots, s_{r}\right)$ se prolonge holomorphiquement au produit des demi-plans $\sigma_{i} \geq-c / r$ et $y$ vérifie

$$
\left|H\left(s_{1}, \ldots, s_{r}\right)\right| \leq \zeta(2-2 c)^{2^{2 r+2}-2^{r+1}+2^{r-1}} .
$$

Les preuves des Propositions 11 et 12 sont très similaires; nous nous contentons de démontrer cette dernière.

Preuve de la Proposition 12. D'après le Paragraphe 1.6.2, on a pour $\sigma_{1}>0, \ldots, \sigma_{r}>0$ 


$$
\begin{aligned}
F\left(s_{1}, \ldots, s_{r}\right) & =\prod_{p}\left(1+\frac{1}{p} P_{r}^{*}\left(p^{-s_{1}}, \ldots, p^{-s_{r}}\right)\right) \\
& =\prod_{p} Q_{r}\left(p^{-s_{1}}, \ldots, p^{-s_{r}} ; p^{-1}\right) \cdot \frac{\prod_{|I| \text { impair }}\left(1-p^{-1} p^{-S_{I}}\right)}{\prod_{P \neq \emptyset,|P| \text { pair }}\left(1-p^{-1} p^{\left.-S_{P}\right)}\right.} \\
& =\frac{\prod_{P \neq \emptyset,|P| \text { pair }} \zeta\left(1+S_{P}\right)}{\prod_{|I| \text { impair }} \zeta\left(1+S_{I}\right)} \cdot H\left(s_{1}, \ldots, s_{r}\right) .
\end{aligned}
$$

Chaque facteur eulérien $Q_{r}\left(p^{-s_{1}}, \ldots, p^{-s_{r}} ; p^{-1}\right)$ est une fonction entière de $\left(s_{1}, \ldots, s_{r}\right)$. Pour montrer le prolongement holomorphe de $\prod_{p} Q_{r}\left(p^{-s_{1}}, \ldots\right.$, $\left.p^{-s_{r}} ; p^{-1}\right)$ au produit des demi-plans $\sigma_{i} \geq-c / r$, il suffit de montrer que dans ce domaine on a

$$
\left|Q_{r}\left(p^{-s_{1}}, \ldots, p^{-s_{r}} ; p^{-1}\right)-1\right| \leq u_{p}
$$

avec $\sum_{p} u_{p}<+\infty$.

Nous supposons donc maintenant que $\sigma_{i} \geq-c / r$ pour $i=1, \ldots, r$, d'où $\left|p^{-s_{i}}\right| \leq p^{c / r}$ pour $i=1, \ldots, r$. On a

$$
\frac{1}{p}\left(\left(1+p^{c / r}\right)^{r}-1\right) \leq 2^{r} p^{c-1} \leq \frac{1}{2}, \quad \text { dès que } \quad p \geq K:=2^{(r+1) /(1-c)} .
$$

Pour $p \geq K$, le (B) de la Proposition 10 nous donne

$$
\left|\log \mathcal{Q}_{r}\left(p^{-s_{1}}, \ldots, p^{-s_{r}} ; p^{-1}\right)\right| \leq \frac{2^{2 r} p^{2 c}}{\frac{1}{2} \cdot p^{2}}=\frac{2^{2 r+1}}{p^{2-2 c}} .
$$

Comme $2-2 c>1$, cela démontre le prolongement holomorphe.

Pour $p<K$, nous utilisons le $(\mathrm{A})$ de la Proposition 10 :

$$
\begin{aligned}
\mid Q_{r}\left(p^{-s_{1}}, \ldots\right. & \left., p^{-s_{r}} ; p^{-1}\right) \mid \\
& \leq\left(1-\frac{1}{p}+\frac{1}{p}\left(1+p^{c / r}\right)^{r}\right) \frac{\prod_{0<2 i \leq r}\left(1+p^{-1+2 i c / r}\right)^{(r} \begin{array}{c}
{ }_{2 i} \\
)
\end{array}}{\prod_{0<2 j-1 \leq r}\left(1-p^{-1+(2 j-1) c / r}\right)^{\left(\begin{array}{c}
r \\
2 j-1
\end{array}\right)}} \\
& \leq\left(1+\frac{2^{r}}{p^{1-c}}\right)\left(1+\frac{1}{p^{1-c}}\right)^{2^{r-1}-1}\left(1-\frac{1}{p^{1-c}}\right)^{-2^{r-1}} \\
& =\left(1+\frac{2^{r}}{p^{1-c}}\right)\left(1+\frac{1}{p^{1-c}}\right)^{2^{r-1}}\left(1-\frac{1}{p^{2-2 c}}\right)^{-2^{r-1}} \\
& \leq\left(1+\frac{2^{2 r+1}}{p^{2-2 c}}\right)\left(1+\frac{2^{r+1}}{p^{2-2 c}}\right)^{2^{r}-1}\left(1-\frac{1}{p^{2-2 c}}\right)^{-2^{r-1}} \\
& \leq\left(1+\frac{1}{p^{2-2 c}}\right)^{2^{2 r+2}-2^{r+1}}\left(1-\frac{1}{p^{2-2 c}}\right)^{-2^{r-1}},
\end{aligned}
$$

où l'on a utilisé que 


$$
p<K \Rightarrow \frac{1}{p^{1-c}}<\frac{2^{r+1}}{p^{2-2 c}} .
$$

On obtient donc

$$
\begin{aligned}
\left|H\left(s_{1}, \ldots, s_{r}\right)\right| & \leq \prod_{p<K}\left(1+\frac{1}{p^{2-2 c}}\right)^{2^{2 r+2}-2^{r+1}}\left(1-\frac{1}{p^{2-2 c}}\right)^{-2^{r-1}} \prod_{p \geq K} \exp \frac{2^{2 r+1}}{p^{2-2 c}} \\
& \leq\left(\prod_{p} \frac{1}{1-\frac{1}{p^{2-2 c}}}\right)^{2^{2 r+2}-2^{r+1}+2^{r-1}} \\
& =\zeta(2-2 c)^{2^{2 r+2}-2^{r+1}+2^{r-1}} .
\end{aligned}
$$

Dans la seconde partie de ce travail, nous aurons également besoin de la précision suivante.

Proposition 13. Soit

$$
H\left(s_{1}, \ldots, s_{r}\right)=\prod_{p} Q_{r}\left(p^{-s_{1}}, \ldots, p^{-s_{r}} ; p^{-1}\right)=: \sum_{n_{i} \geq 1} \frac{h\left(n_{1}, \ldots, n_{r}\right)}{n_{1}^{s_{1}} \cdots n_{r}^{s_{r}}}
$$

la fonction définie à la Proposition 12. Pour tout c tel que $0<c<1 / 2$, la fonction

$$
\widetilde{H}\left(s_{1}, \ldots, s_{r}\right):=\sum_{n_{i} \geq 1} \frac{\left|h\left(n_{1}, \ldots, n_{r}\right)\right|}{n_{1}^{s_{1}} \cdots n_{r}^{s_{r}}}
$$

se prolonge holomorphiquement au produit des demi-plans $\sigma_{i} \geq-c / r$.

Preuve. Les fonctions entières $\widetilde{Q}\left(p^{-s_{1}}, \ldots, p^{-s_{r}}\right)$ de $\left(s_{1}, \ldots, s_{r}\right)$ définies par $\widetilde{H}\left(s_{1}, \ldots, s_{r}\right)=: \prod_{p} \widetilde{Q}\left(p^{-s_{1}}, \ldots, p^{-s_{r}}\right)$ ne sont pas aisées à déterminer. Cependant, en évaluant la taille (en valeur absolue) des coefficients de $p^{-j_{1} s_{1}-\cdots-j_{r} s_{r}}$ obtenus en développant le produit

$$
\begin{aligned}
Q_{r}\left(p^{-s_{1}}, \ldots, p^{-s_{r}} ; p^{-1}\right) & \\
= & \left(1+\sum_{|P| \neq 0 \text { pair }} \frac{1}{p^{1+S_{P}}}-\sum_{|I| \text { impair }} \frac{1}{p^{1+S_{I}}}\right) \\
& \times \prod_{|P| \neq 0 \text { pair }}\left(1-\frac{1}{p^{1+S_{P}}}\right) \prod_{|I| \text { impair }}\left(1+\frac{1}{p^{1+S_{I}}}+\frac{1}{p^{2+2 S_{I}}}+\cdots\right) \\
= & \sum_{j_{i} \geq 0} \frac{h\left(p^{j_{1}}, \ldots, p^{j_{r}}\right)}{p^{j_{1} s_{1}+\cdots+j_{r} s_{r}}},
\end{aligned}
$$

on établit, si $0<c<1 / 2$ et $\sigma_{i} \geq-c / r$, la majoration

$$
\left|\sum_{j_{i} \geq 0 \text { non tous nuls }} \frac{\left|h\left(p^{j_{1}}, \ldots, p^{j_{r}}\right)\right|}{p^{j_{1} s_{1}+\cdots+j_{r} s_{r}}}-1\right| \ll \frac{1}{p^{1+(1-2 c)}},
$$

d'où suit immédiatement la proposition. Soit $j$ le plus grand des $j_{i}$. 
(1) Si $j=1$ alors nous nous intéressons au coefficient d'un certain $p^{-s_{n_{1}}-\cdots-s_{n_{t}}}$, pour un $t$ avec $1 \leq t \leq r$, calculé en développant le produit (6). Si $t$ est pair, alors $s_{n_{1}}+\cdots+s_{n_{t}}=S_{P_{0}}$ pour un certain $P_{0} \neq \emptyset$ avec $\left|P_{0}\right|$ pair. Outre la contribution nulle $1 / p-1 / p$ à ce coefficient ne faisant intervenir qu'un seul terme non égal à 1 dans les différents facteurs de (6), les éventuelles autres contributions, forcément en nombre fini, faisant intervenir au moins deux termes non égaux à 1 dans ces facteurs, sont de la forme $\pm 1 / p^{n}$ pour $n=2$ ou 3. L'argument est tout à fait similaire si $t$ est impair. Donc dans ce cas le coefficient cherché est, en valeur absolue, $\ll p^{-2}$.

(2) Si $j \geq 2$ alors n'importe laquelle des contributions — qui sont en nombre fini - au coefficient de $p^{-j_{1} s_{1}-\cdots-j_{r} s_{r}}$ obtenues en développant le produit (6) est de la forme $\pm 1 / p^{j+n}$, pour des $n \geq 0$. Donc dans ce cas le coefficient cherché est, en valeur absolue, $\ll p^{-j}$.

Par symétrie nous pouvons nous contenter de considérer le cas où $j_{1} \geq$ $\cdots \geq j_{r}$ afin d'estimer le terme à gauche de (7), qui est donc

$$
\begin{aligned}
& \ll \sum_{j_{i} \leq j_{1}=1} \frac{1}{p^{j_{1} \sigma_{1}+\cdots+j_{r} \sigma_{r}+2}}+\sum_{j_{1} \geq 2} \frac{1}{p^{j_{1} \sigma_{1}}} \cdots \sum_{j_{r-1} \leq j_{1}} \frac{1}{p^{j_{r-1} \sigma_{r-1}}} \sum_{j_{r} \leq j_{1}} \frac{1}{p^{j_{r} \sigma_{r}+j_{1}}} \\
& \ll \sum_{j_{i} \leq j_{1}=1} \frac{1}{p^{-c+2}}+\sum_{j_{1} \geq 2} \frac{1}{p^{-c j_{1} / r}} \cdots \sum_{j_{r-1} \leq j_{1}} \frac{1}{p^{-c j_{r-1} / r}} \sum_{j_{r} \leq j_{1}} \frac{1}{p^{-c j_{r} / r+j_{1}}} \\
& \ll \frac{1}{p^{2-c}}+\sum_{\substack{j_{1} \geq 2 \\
j_{i} \leq j_{1}}} \frac{1}{p^{j_{1}(1-c)}} \ll \frac{1}{p^{2(1-c)}}
\end{aligned}
$$

lorsque $\sigma_{i} \geq-c / r$ avec $0<c<1 / 2(i=1, \ldots, r)$.

1.6.5. Estimation de l'erreur de troncature. Soit $S_{r}(x)$ comme en (1), et définissons $E_{r}(x)=0$ si $x$ n'est pas entier, et

$$
E_{r}(x):=\sum_{n_{j} \leq x(2 \leq j \leq r)} \frac{\left|\mu(x) \mu\left(n_{2}\right) \cdots \mu\left(n_{r}\right)\right|}{\left[x, n_{2}, \ldots, n_{r}\right]}
$$

si $x$ est entier. Notons que

$$
\left|S_{r}(x)-\sum_{n_{j} \leq x}^{\prime} \frac{\mu\left(n_{1}\right) \cdots \mu\left(n_{r}\right)}{\left[n_{1}, \ldots, n_{r}\right]}\right| \ll E_{r}(x) .
$$

Posons

$$
F\left(s_{1}, \ldots, s_{r}\right):=\sum_{n_{1}, \ldots, n_{r}} \frac{\mu\left(n_{1}\right) \cdots \mu\left(n_{r}\right)}{\left[n_{1}, \ldots, n_{r}\right] n_{1}^{s_{1}} \cdots n_{r}^{s_{r}}}
$$


et

$$
I:=\frac{1}{(2 \pi i)^{r}} \int_{\kappa-i T}^{\kappa+i T} \int_{N_{2}(\kappa-i T)}^{N_{2}(\kappa+i T)} \cdots \int_{N_{r}(\kappa-i T)}^{N_{r}(\kappa+i T)} F\left(s_{1}, \ldots, s_{r}\right) x^{s_{1}+\cdots+s_{r}} \frac{d s_{1} \cdots d s_{r}}{s_{1} \cdots s_{r}}
$$

où $\kappa=(\log x)^{-1}$ et $1 \leq N_{2} \leq \cdots \leq N_{r}$. Alors par les Propositions 6 et 12 (où nous posons $s=\sigma=0$, et où nous avons remplacé les symboles $w_{i}$ par $\left.s_{i}, i=1, \ldots, r\right)$ nous avons

$$
S_{r}(x)-I \ll F_{r}^{\odot}(\kappa, x, T)+E_{r}(x),
$$

avec

$$
F\left(s_{1}, \ldots, s_{r}\right)=\frac{\prod_{P \neq \emptyset,|P| \text { pair }} \zeta\left(1+S_{P}\right)}{\prod_{|I| \text { impair }} \zeta\left(1+S_{I}\right)} \cdot H\left(s_{1}, \ldots, s_{r}\right),
$$

pour une fonction $H$ régulière et bornée lorsque toutes les variables $s_{i}$ satisfont $\Re s_{i} \geq-c$, où $c=c(r)$ est une constante inférieure à $1 /(2 r)$ (par exemple $c=1 /(2 r+1)$ convient), et où l'ordre de grandeur mesurant l'erreur de troncature satisfait

$$
F_{r}^{\odot}(\kappa, x, T):=\sum_{n_{1}, \ldots, n_{r}} \frac{\left|\mu\left(n_{1}\right) \cdots \mu\left(n_{r}\right)\right|}{\left[n_{1}, \ldots, n_{r}\right]\left(n_{1} \cdots n_{r}\right)^{\kappa} \min _{j=1, \ldots, r}\left(T\left|\log \left(x / n_{j}\right)\right|+1\right)} .
$$

Nous posons $\log T=C(\log x)^{3 / 5}(\log \log x)^{-1 / 5}$ pour une constante positive $C$. Les quantités $N_{1}=M_{1}, \ldots, N_{r}=M_{r}$ sont des constantes positives (satisfaisant les hypothèses de la Proposition 6) qui seront définies dans la deuxième partie de ce travail, au Paragraphe 2.9.

Nous allons maintenant montrer que la fonction en (13) mesurant l'erreur de troncature satisfait

$$
F_{r}^{\bigcirc}(\kappa, x, T) \ll \delta(x),
$$

et que la fonction en (8) mesurant l'erreur en (9) satisfait

$$
E_{r}(x) \ll x^{-1+O(1 / \log \log x)} .
$$

Les cinq lemmes qui suivent seront utiles.

LEMME 1. Si $k>1$ est un entier, alors

$$
\begin{aligned}
\sum_{n \leq x} d(n)^{k}= & x\left(A_{1}(\log x)^{2^{k}-1}+A_{2}(\log x)^{2^{k}-2}+\cdots+A_{k}\right) \\
& +O\left(x^{\left(2^{k}-1\right) /\left(2^{k}+2\right)+\varepsilon}\right),
\end{aligned}
$$

où $d(n)$ désigne la fonction "nombre de diviseurs" de Dirichlet, et où les $A_{j}$ sont des constantes réelles. 
Ramanujan $[\mathrm{R}]$ a énoncé cette formule en 1916, sous l'hypothèse de Riemann, avec la meilleure estimation de l'erreur $O\left(x^{1 / 2+\varepsilon}\right)$; B. M. Wilson [W] l'a démontrée en 1923 (sans supposer l'hypothèse de Riemann).

Lemme 2. Soit $I_{x, T}$ l'ensemble des entiers $n$ satisfaisant $x / T \leq|n-x|$ $\leq x / 2$. Si $k>1$ est un entier, alors

$$
\sum_{n \in I_{x, T}} \frac{d(n)^{k}}{|n-x|} \ll(\log x)^{2^{k}}
$$

Preuve. Nous définissons la quantité $K$ par

$$
(1+1 / T)^{K-1} \leq 2<(1+1 / T)^{K},
$$

et notons $\beta:=1+1 / T$. L'autre partie de la somme s'estimant de façon similaire, nous nous contentons de considérer les termes $\sum_{x / T \leq n-x \leq x / 2} \frac{d(n)^{k}}{n-x}$. Ils sont

$$
\begin{aligned}
& \ll \sum_{i=1}^{K-1} \sum_{x \beta^{i} \leq n<x \beta^{i+1}} \frac{d(n)^{k}}{n-x} \\
& \ll \frac{1}{x} \sum_{i=1}^{K} \frac{1}{\beta^{i}-1} \sum_{x \beta^{i} \leq n<x \beta^{i+1}} d(n)^{k} \ll \sum_{i=1}^{K} \frac{\beta^{i}}{T\left(\beta^{i}-1\right)}(\log x)^{2^{k}-1},
\end{aligned}
$$

où pour la dernière majoration nous avons utilisé le Lemme 1. En remarquant que $\beta^{i}-1 \gg i / T$ et que $\beta^{i} \ll 1$, nous voyons que cette dernière somme est

$$
\ll \sum_{i=1}^{K} \frac{1}{i}(\log x)^{2^{k}-1} \ll(\log x)^{2^{k}} .
$$

Lemme 3. Soit $I_{x}$ l'ensemble des entiers $n$ satisfaisant $|n-x| \leq x / 2$. Si $k>1$ est un entier, alors

$$
\sum_{n \in I_{x}} \frac{d(n)^{k}}{1+T|\log (x / n)|} \ll \frac{x}{T}(\log x)^{2^{k}} .
$$

Preuve. La somme à estimer est

$$
\ll \sum_{|n-x| \leq x / T} d(n)^{k}+\frac{x}{T} \sum_{n \in I_{x, T}} \frac{d(n)^{k}}{|n-x|} \ll \frac{x}{T}(\log x)^{2^{k}-1}+\frac{x}{T}(\log x)^{2^{k}},
$$

où l'on a fait appel aux Lemmes 1 et 2 .

Lemme 4. Soit $a_{1}=q_{1} \cdots q_{l}$, où $q_{1}, \ldots, q_{l}$ sont des nombres premiers distincts. Si $r>1$ est un entier, alors

$$
\sum_{n_{2}, \ldots, n_{r}} \frac{\left|\mu\left(n_{2}\right) \cdots \mu\left(n_{r}\right)\right|\left\langle a_{1},\left[n_{2}, \ldots, n_{r}\right]\right\rangle}{\left[n_{2}, \ldots, n_{r}\right]\left(n_{2} \cdots n_{r}\right)^{\kappa}} \ll d\left(a_{1}\right)^{r-1}(\log x)^{2^{r-1}-1} .
$$


Preuve. Si l'on note $g\left(a_{1}, n_{2}, \ldots, n_{r}\right)$ le terme général à sommer, on vérifie facilement que $g$ est multiplicative. La Proposition 2 s'applique donc, et la somme à évaluer peut s'écrire

$$
\begin{aligned}
\prod_{p} \sum_{0 \leq j_{i} \leq 1} \frac{\left|\mu\left(p^{j_{2}}\right) \cdots \mu\left(p^{j_{r}}\right)\right|\left\langle a_{1},\left[p^{j_{2}}, \ldots, p^{j_{r}}\right]\right\rangle}{\left[p^{j_{2}}, \ldots, p^{j_{r}}\right]\left(p^{j_{2}} \cdots p^{j_{r}}\right)^{\kappa}} \\
\quad=\prod_{p \mid a_{1}}\left(\sum_{i=0}^{r-1}\left(\begin{array}{c}
r-1 \\
i
\end{array}\right) p^{-i \kappa}\right) \prod_{p \nmid a_{1}}\left(1+\sum_{i=1}^{r-1}\left(\begin{array}{c}
r-1 \\
i
\end{array}\right) p^{-i \kappa-1}\right) \\
\leq \prod_{p \mid a_{1}}\left(1+\frac{1}{p^{\kappa}}\right)^{r-1} \prod_{p \nmid a_{1}}\left(1+\frac{2^{r-1}-1}{p^{\kappa+1}}\right) \\
\leq d\left(a_{1}\right)^{r-1} \zeta(1+\kappa)^{2^{r-1}-1} \ll d\left(a_{1}\right)^{r-1}(\log x)^{2^{r-1}-1},
\end{aligned}
$$

où $p$ parcourt l'ensemble des nombres premiers, et où dans la dernière estimation on a utilisé la majoration $\zeta(1+\kappa) \ll 1 / \kappa$.

Lemme 5. Soit $x=q_{1} \cdots q_{l}$, où $q_{1}, \ldots, q_{l}$ sont des nombres premiers distincts. Si $r>1$ est un entier, alors

$$
\sum_{n_{2} \leq x, \ldots, n_{r} \leq x} \frac{\left|\mu\left(n_{2}\right) \cdots \mu\left(n_{r}\right)\right|\left\langle x,\left[n_{2}, \ldots, n_{r}\right]\right\rangle}{\left[n_{2}, \ldots, n_{r}\right]} \ll x^{O(1 / \log \log x)} .
$$

Preuve. La somme à estimer est

$$
\begin{aligned}
& \leq \sum_{p \mid n_{j} \Rightarrow p \leq x} \frac{\left|\mu\left(n_{2}\right) \cdots \mu\left(n_{r}\right)\right|\left\langle x,\left[n_{2}, \ldots, n_{r}\right]\right\rangle}{\left[n_{2}, \ldots, n_{r}\right]} \\
& =\prod_{p \mid x}\left(\sum_{i=0}^{r-1}\left(\begin{array}{c}
r-1 \\
i
\end{array}\right)\right) \prod_{p \nmid x, p<x}\left(1+\sum_{i=1}^{r-1}\left(\begin{array}{c}
r-1 \\
i
\end{array}\right) p^{-1}\right) .
\end{aligned}
$$

(L'égalité ci-dessus s'obtient de façon similaire à l'égalité (16), en notant que cette dernière reste vraie, pour des produits restreints aux $p \leq a_{1}=x$, lorsque $\kappa=0$.) La somme à estimer est donc

$$
\leq 2^{(r-1) \omega(x)} \prod_{p<x}\left(1+\frac{2^{r-1}-1}{p}\right) \leq 2^{(r-1) \omega(x)} \prod_{p<x}\left(1+\frac{1}{p}\right)^{2^{r-1}-1},
$$

où $\omega(n)$ désigne le nombre de facteurs premiers distincts de $n$. L'estimation voulue (et même une estimation plus précise) est obtenue en rappelant que, d'une part $\lim \sup \varpi(x)=1$, où $\varpi(x):=\omega(x) \log \log x / \log x$ (puisque, pour des $\alpha_{i} \geq 1$ et des nombres premiers $p_{i}$, on a toujours $\varpi\left(p_{1}^{\alpha_{1}} \cdots p_{l}^{\alpha_{l}}\right)$ $\left.\leq \varpi\left(p_{1} \cdots p_{l}\right)+O(1) \leq \varpi\left(\prod_{p \leq y, \pi(y)=l} p\right)+O(1)\right)$, d'autre part que $\prod_{p<x}(1+1 / p) \ll \log x$. 
Preuve de (14) et (15). Commençons par considérer $F_{r}^{\odot}(\kappa, x, T)$. Nous répartissons les termes de la somme (13) à estimer en deux sommes $\sum_{1}$ et $\sum_{2}$, la première comprenant tous les termes pour lesquels tous les $n_{i}$ sont en dehors de $I_{x}$. Par la Proposition 11 (avec $c=1 / 4$, par exemple) nous avons

$$
\begin{aligned}
\sum_{1} & \ll \frac{1}{T} \sum_{n_{1}, \ldots, n_{r}} \frac{\left|\mu\left(n_{1}\right) \cdots \mu\left(n_{r}\right)\right|}{\left[n_{1}, \ldots, n_{r}\right]\left(n_{1} \cdots n_{r}\right)^{\kappa}} \\
& \leq \frac{1}{T} \mathcal{G}(\kappa, \ldots, \kappa) \prod_{E \neq \emptyset} \zeta(1+\kappa) \ll \frac{1}{T}(\log x)^{2^{r}-1} .
\end{aligned}
$$

D'autre part, si l'on note que, par symétrie, $\sum_{2} \leq \sum_{2,1}+\cdots+\sum_{2, r} \ll \sum_{2,1}$, où la somme $\sum_{2, i}$ comprend tous les termes de $\sum_{2}$ satisfaisant chacune des deux conditions $n_{i} \in I_{x}$ et $\min _{j=1, \ldots, r}\left(T\left|\log \left(x / n_{j}\right)\right|+1\right)=T\left|\log \left(x / n_{i}\right)\right|+1$, nous voyons que

$$
\begin{aligned}
\sum_{2} & \ll \sum_{2,1} \\
& \leq \sum_{n_{1} \in I_{x}} \frac{\left|\mu\left(n_{1}\right)\right|}{n_{1}^{1+\kappa}\left(1+T\left|\log \left(x / n_{1}\right)\right|\right)} \sum_{n_{2}, \ldots, n_{r}} \frac{\left|\mu\left(n_{2}\right) \cdots \mu\left(n_{r}\right)\right|\left\langle n_{1},\left[n_{2}, \ldots, n_{r}\right]\right\rangle}{\left[n_{2}, \ldots, n_{r}\right]\left(n_{2} \cdots n_{r}\right)^{\kappa}} .
\end{aligned}
$$

Par les Lemmes 4 puis 3 nous avons donc

$$
\sum_{2} \ll \frac{(\log x)^{2^{r-1}-1}}{x} \sum_{n \in I_{x}} \frac{d(n)^{r-1}}{1+T|\log (x / n)|} \ll \frac{1}{T}(\log x)^{2^{r}-1} \ll \delta(x),
$$

ce qui termine la démonstration de (14).

Passons à la preuve de (15). Nous pouvons clairement supposer que $x$ est un entier sans facteur carré. Par le Lemme 5 nous avons donc

$$
\begin{aligned}
E_{r}(x) & =\frac{1}{x} \sum_{n_{2} \leq x, \ldots, n_{r} \leq x} \frac{\left|\mu(x) \mu\left(n_{2}\right) \cdots \mu\left(n_{r}\right)\right|\left\langle x,\left[n_{2}, \ldots, n_{r}\right]\right\rangle}{\left[n_{2}, \ldots, n_{r}\right]} \\
& \ll x^{-1+O(1 / \log \log x)} .
\end{aligned}
$$

Par (12) nous avons donc obtenu le résultat principal de cette première partie.

ThÉORÈme 2. Si $S_{r}(x)$ est définie par (1) et I par (11), nous avons

$$
S_{r}(x)-I \ll \delta(x) .
$$

En conclusion à cette première partie nous rappelons également sous la forme d'un théorème un résultat auxiliaire, qui est énoncé dans les Propositions 12 et 13 ci-dessus, et que nous utiliserons plus bas.

THÉORÈME 3. Nous avons

$$
F\left(s_{1}, \ldots, s_{r}\right)=\frac{\prod_{P \neq \emptyset,|P| \text { pair }} \zeta\left(1+S_{P}\right)}{\prod_{|I| \text { impair }} \zeta\left(1+S_{I}\right)} \cdot H\left(s_{1}, \ldots, s_{r}\right),
$$

pour une fonction $H=H_{r}$ représentée par une série de Dirichlet, et pour 
laquelle $\widetilde{H}$ (définie en $(5))$ est régulière lorsque toutes les variables $s_{i}$ satisfont $\sigma_{i}>-1 /(2 r)$, et bornée si elles satisfont $\sigma_{i} \geq-c$, où $c=c(r)$ est une constante inférieure à $1 /(2 r)$ (par exemple $c=1 /(2 r+1)$ convient).

\section{DEUXIÈME PARTIE DÉMONSTRATION DU THÉORÈME 1}

2.1. Remerciements. Michel Balazard a été à l'origine de ce projet, auquel il a commencé à travailler en 1999 déjà, et auquel nous ne nous sommes joints que deux ou trois années après. Sans lui cet article n'aurait jamais vu le jour. Mais Michel a finalement tenu, malgré notre insistance et à notre grand regret, à se retirer de la deuxième partie de ce travail. Il a en effet longtemps espéré pouvoir offrir une version plus simple de la démonstration que cette partie contient, et la version présente ne satisfait pas son goût de simplicité et d'élégance. "Des remerciements me suffiraient amplement", ditil. Nous l'assurons donc évidemment de toute notre gratitude; mais est-ce réellement suffisant?

2.2. Notations. On considérera dans la suite les vecteurs $s=\left[s_{1}, \ldots, s_{r}\right]$ $=\left[\begin{array}{c}s_{1} \\ \vdots \\ s_{r}\end{array}\right]$ de $\mathbb{R}^{r}$, où les $s_{i}$ sont les variables décrites précédemment, ainsi que des vecteurs $v$ constants de $\mathbb{R}^{r}, v=\left[w_{1}, \ldots, w_{r}\right]=\left[\begin{array}{c}w_{1} \\ \vdots \\ w_{r}\end{array}\right]$. Le choix de l'écriture de ces vecteurs comme matrices $1 \times r$ ("en ligne") ou $r \times 1$ "en colonne" sera imposé naturellement par le contexte, en particulier lorsque ces vecteurs interviendront dans un produit matriciel.

On notera $\mathcal{U}=\mathcal{U}_{r}$ l'ensemble des vecteurs de $\mathbb{R}^{r}$ dont chaque composante est 0 ou 1, dont on considérera également les sous-ensembles suivants :

- $\mathcal{U}_{P}$ : les vecteurs dont le nombre de composantes égales à 1 est pair positif;

- $\mathcal{U}_{I}$ : ceux dont le nombre de composantes égales à 1 est impair;

- $\mathcal{U}_{I}^{*}$ : ceux dont le nombre de composantes égales à 1 est impair et supérieur à 1 .

On notera également $v_{0}$ le vecteur (de $\mathcal{U}_{I}$ si $r$ est impair et de $\mathcal{U}_{P}$ sinon) dont toutes les $r$ composantes sont égales à 1 , et $\mathcal{O}$ le vecteur nul.

Nous pouvons donc récrire ainsi l'intégrale $I$ définie ci-dessus en (11) :

$$
I=\frac{1}{(2 \pi i)^{r}} \int_{\kappa-i T}^{\kappa+i T} \int_{N_{2}(\kappa-i T)}^{N_{2}(\kappa+i T)} \cdots \int_{N_{r}(\kappa-i T)}^{N_{r}(\kappa+i T)} \frac{\prod_{v \in \mathcal{U}_{P}} \zeta(1+v s)}{\prod_{v \in \mathcal{U}_{I}^{*}} \zeta(1+v s)} G_{r}(s) x^{v_{0} s} d s_{1} \cdots d s_{r},
$$


où

$$
G_{r}(s):=H_{r}(s) \prod_{i=1}^{r} \frac{1}{\zeta\left(1+s_{i}\right) s_{i}}
$$

Remarque 3. Les facteurs $1 / \zeta\left(1+s_{i}\right)$ et $1 / s_{i}$ ont été incorporés à la fonction $G_{r}$, en vue d'un argument récursif faisant intervenir les singularités de l'intégrand, puisque les premiers "tuent" les pôles des seconds en $s_{i}=0$. Les facteurs $1 / s_{i}$ sont cependant essentiels, et nous les ferons réapparaître au Paragraphe 2.5 pour la preuve du Théorème 1.

\subsection{Structure de la démonstration}

2.3.1. Plan. L'évaluation de l'intégrale $I$ se fait à l'aide d'applications répétées du théorème des résidus : à chaque étape du processus, l'intégrale intérieure est remplacée par une somme de résidus et un terme de contribution négligeable. La nouvelle intégrale intérieure de chaque résidu est alors considérée séparément : la mise en place du mécanisme de récurrence utilisé nécessite donc des permutations de variables, associées à d'autres manipulations, sur lesquelles, dans un souci de clarté, nous avons choisi de nous étendre assez longuement dans ce qui suit.

Ainsi ce Paragraphe 2.3 est entièrement consacré à une description générale de ce mécanisme de récurrence. De plus, les deux premières étapes de l'évaluation étant, par leur trop grande simplicité, impropres à l'illustration de toutes les difficultés d'une étape quelconque, nous avons donné au Paragraphe 2.6 une description détaillée des trois premières étapes. Ceci nous permet d'introduire d'autre part les notations requises pour le cas général, qui est traité aux Paragraphes 2.7 et 2.7.1.

Les Paragraphes 2.4, 2.5, 2.8 et 2.9 contiennent divers résultats auxiliaires. La démonstration du Théorème 1 est achevée au Paragraphe 2.10.

2.3.2. Le mécanisme de récurrence : description générale. Nous choisissons a priori un ordre pour l'évaluation des intégrales successives de $I$, par rapport aux variables $s_{r}, s_{r-1}, \ldots, s_{1}$.

REMARQUE 4. L'ordre des variables encore non intégrées est susceptible d'être modifié à tout moment en cours d'évaluation, ces modifications dépendant du terme particulier de $I$ que l'on est en train de calculer.

L’idée générale est de remplacer successivement chaque intégrale intérieure par une somme de résidus exprimés en termes des variables non encore intégrées : la variable en cours de traitement est donc remplacée par une combinaison linéaire des variables non encore considérées. Soit $s_{n_{r}}=s_{r}, s_{n_{r-1}}, \ldots, s_{n_{1}}$ une permutation des variables adéquate au traitement d'un terme de la $m+1$-ème étape $(m \geq 0)$. À ce moment, nous serons 
en présence d'une intégrale $r-m$-uple de la forme

$$
\begin{aligned}
& P_{m}(\log x) \\
& \times \int_{N_{n_{1}} \kappa-i K^{-q} T}^{N_{n_{1}} \kappa+i K^{-q} T} \frac{1}{\zeta\left(1+s_{n_{1}}\right) s_{n_{1}}} \cdots \int_{N_{n_{r-m}} \kappa-i K^{r-m-1-q} T}^{N_{n_{r-m}} \kappa+i K^{r-m-1-q} T} \frac{1}{\zeta\left(1+s_{n_{r-m}}\right) s_{n_{r-m}}} \\
& \times \prod_{i=r-m+1}^{r} \eta^{\left(a_{i, m}\right)}\left(T_{(m)} s_{n_{i}}\right) \prod_{v \in \mathcal{U}_{P} \backslash V_{m}} \zeta\left(P, m, v, i_{n_{2}}, \ldots, i_{n_{m}}, s\right) \\
& \times \prod_{v \in \mathcal{U}_{I}^{*} \backslash V_{m}} \zeta\left(I, m, v, j_{n_{2}}, \ldots, j_{n_{m}}, s\right) H_{r-m}(s) x^{T_{(m)} v_{0} s} d s_{n_{r-m}} \cdots d s_{n_{1}},
\end{aligned}
$$

où $P_{m}$ est un polynôme, où $s_{n_{i}}(i=1, \ldots, r)$ est une permutation (dépendant du terme considéré, et bien sûr de $m)$ des variables $s_{j}(j=1, \ldots, r)$ où $K>2$ est une constante (voir (63)) et $q$ le nombre d'applications du procédé (b) ci-dessous jusqu'ici, où $T_{(m)}$ est une application linéaire décrite dans le Paragraphe 2.4, où l'on note abusivement $T_{(m)} s_{n_{i}}$ pour

$$
T_{(m)}\left[\begin{array}{c}
0 \\
\vdots \\
0 \\
s_{n_{i}} \\
0 \\
\vdots \\
0
\end{array}\right]
$$

( $s_{n_{i}}$ étant le $i$-ème coefficient du vecteur), où $H_{r-m}(s)$ est une fonction des variables $s_{n_{i}}(i=1, \ldots, r-m)$, régulière et bornée pour $\left|\sigma_{n_{i}}\right| \geq c$, avec $c=c(r)>0$, où $\eta(s):=(\zeta(1+s) s)^{-1}$, et où les fonctions $\zeta(P, \ldots)$ et $\zeta(I, \ldots)$ sont définies récursivement (voir Paragraphe 2.7) à partir de $\zeta\left(1+T_{1} v s\right)=: \zeta(P, 1, v, s)$ et de $\zeta\left(1+T_{1} v s\right)^{-1}=: \zeta(I, 1, v, s), T_{1}$ étant l'application linéaire décrite au Paragraphe 2.4.

(a) À une telle $m+1$-ème étape, et ceci pour autant que le coefficient $c_{n_{r-m}}$ de $s_{n_{r-m}}$ dans l'exposant du facteur $x^{\sum_{j \leq r-m} c_{n_{j}} s_{n_{j}}}$ de l'intégrand (provenant de $x^{v_{0} s}$ par une succession de transformations linéaires) soit non nul, nous considérons un des résidus obtenus à l'étape précédente, et nous remplaçons son intégrale intérieure par rapport à la $m$-ème variable $s_{n_{r-m}}$ (qui n'est $s_{r-m}$ que si aucune permutation des variables n'a été encore requise lors des étapes précédentes), par la somme des résidus de certains des pôles de l'intégrand par rapport à $s_{n_{r-m}}$. La variable $s_{n_{r-m}}$ prend certaines valeurs $s_{n_{r-m}}^{*}$, qui sont des combinaisons linéaires des $s_{n_{j}}(j \leq r-m-1)$. 
Les pôles considérés sont ceux qui se situent à l'intérieur d'un rectangle dont l'un des côtés est le segment d'abscisse d'intégration de la variable $s_{n_{r-m}}$ (segment éventuellement modifié à une étape antérieure si une permutation des variables a été nécessaire, soit si le procédé (b) ci-dessous a déjà été utilisé). Nous montrons (au Lemme 18) que la contribution à $I$ de l'intégrale intérieure sur les trois autres côtés de ce rectangle est $\ll T^{-1+\varepsilon}=\delta(x)$ (et donc que dans le cas où l'intégrand n'a pas de pôle par rapport à la variable $s_{n_{r-m}}$, la contribution totale associée à ce terme est $\ll T^{-1+\varepsilon}=\delta(x)$ ). Quant à chacun des termes correspondants aux résidus obtenus dans cette opération, il a une forme semblable à celle dont nous sommes partis, soit de type (18) à nouveau, mais avec $m+1$ à la place de $m$.

Les abscisses d'intégration $K^{j-1} \kappa$ (où donc $K=N_{2}$ ), satisfaisant $N_{j} \kappa=$ $N_{2}^{j-1}$, seront choisies (voir le Paragraphe 2.9) de façon à éviter que les chemins d'intégration des intégrales non encore évaluées nous fassent passer, lorsque nous intégrons sur l'abscisse d'intégration de $s_{n_{r-m}}$, trop près d'un pôle $s_{n_{r-m}}=s_{n_{r-m}}^{*}$ de l'intégrand. D'autre part, nous voulons également éviter de passer près d'un pôle de l'intégrand lors de la déformation rectangulaire du chemin d'intégration par rapport à $s_{n_{r-m}}$ : nous verrons que le choix des nombres $N_{j}$ donne également cette garantie tant que $s_{n_{r-m}}=s_{r-m}$, c'est-à-dire tant qu'aucune permutation des variables n'a encore été requise. Mais si l'on change l'ordre d'intégration ceci ne peut plus être garanti sans que les longueurs des segments d'abscisses d'intégration soit préalablement modifiées (réduites). Nous procédons alors comme suit.

(b) Si $c_{n_{r-m}}=0$, l'intégrale intérieure ayant des bornes d'intégration de la forme $N_{n_{r-m}} \kappa+i K^{r-m-1-q} T$ où $q$ est le nombre d'applications déjà réalisées de ce procédé (b), alors nous commençons par vérifier (Lemme 19) que la contribution à $I$ de cette intégrale intérieure sur le tronçon d'abscisse de $N_{n_{r-m}} \kappa+i K^{-1-q} T$ à $N_{n_{r-m}} \kappa+i K^{r-m-1-q} T$ est $\ll T^{-1+\varepsilon}=\delta(x)$.

Ensuite, nous permutons les variables et récrivons l'intégrale à évaluer par rapport aux variables $s_{n_{r-m-1}}, \ldots, s_{n_{1}}, s_{n_{r-m}}$, dans l'ordre indiqué, que nous rebaptisons $s_{\nu_{r-m}}, \ldots, s_{\nu_{2}}, s_{\nu_{1}}$, en remplaçant le segment d'intégration par rapport à $s_{n_{r-m}}=s_{\nu_{1}}$ par le tronçon d'abscisse de $N_{n_{r-m}} \kappa-i K^{-1-q} T$ à $N_{n_{r-m}} \kappa+i K^{-1-q} T$ (voir le Paragraphe 2.9 ci-dessous, et en particulier la définition (63)). Le terme ainsi modifié a lui aussi une forme semblable à celle dont nous sommes partis, soit de type (18) à nouveau, mais avec $q+1$ à la place de $q$.

Remarque 5. Ainsi, si l'abscisse d'intégration $N_{k} \kappa$ d'une variable $s_{k}$ lui reste attachée tout au long du procédé d'évaluation de $I$, la longueur du segment d'intégration par rapport à $s_{k}$ peut être (éventuellement à plusieurs reprises) divisée par un facteur positif (de la forme $K^{r-m}$ : voir (63)), lors de permutations des variables. 
Si le nouveau coefficient $c_{n_{r-m}}$ est non nul, on procède comme en (a), sinon, une nouvelle fois comme en (b). Nous parvenons ainsi à l'une des deux situations finales suivantes :

(f1) l'intégrand ne contient plus aucun pôle et $c_{n_{r-m}} \neq 0$;

(f2) tout l'exposant $\sum_{j \leq r-m} c_{n_{j}} s_{n_{j}}$ de $x$ est nul.

(La troisième situation, où après la $r$-1-ème étape le dernier coefficient satisfait $c_{n_{1}} \neq 0$, et le dernier intégrand de la dernière intégrale contient encore au moins un pôle, est démontrée impossible par le Lemme 14.)

Dans le cas (f2) nous vérifions alors que la contribution du terme considéré est de la forme $P_{m}(\log x)+O\left(T^{-1+\varepsilon}\right)$, où $P_{m}$ est un polynôme, ce qui montre le (i) du Théorème 1. (Le fait que dans ce cas (f2) l'intégrand contienne ou ne contienne pas de pôle est sans importance : on ne déforme plus que très faiblement les contours d'intégration pour cette évaluation finale, qui se fait sur des tronçons d'intégration ne pouvant de toute façon contenir aucun des éventuels pôles de l'intégrand : voir le Lemme 20 à la fin de ce travail.) Et nous verrons (Lemme 15) que nous aboutissons toujours au cas (f1) lorsque $r$ est impair, ce qui montre le (ii) du Théorème 1.

2.3.3. Pôles et résidus. Nous commençons donc par appliquer le théorème des résidus à l'intégrale intérieure de (2) (par rapport à la variable $\left.s_{r}\right)$. Nous remplaçons cette intégrale intérieure par une somme de résidus. Nous le faisons pour l'instant "formellement" ; il faudra s'assurer que l'erreur commise associée à cette opération reste négligeable (Lemme 18).

Chaque résidu s'obtient en choisissant un des $s_{r}=s_{r}^{*}\left(s_{1}, \ldots, s_{r-1}\right)$ pour lesquels la fonction $\prod_{v \in \mathcal{U}_{P}} \zeta(1+v s) / \prod_{v \in \mathcal{U}_{I}^{*}} \zeta(1+v s)$ a un pôle, soit, dans cette première étape, pour lesquels l'un des facteurs du dénominateur (pour $v=v_{1}$, mettons) satisfait $v_{1} s=0$. Nous sommes donc en présence de pôles d'ordre 1, pour chacun desquels le résidu s'écrit simplement

$$
R(1)=\frac{\prod_{v \in \mathcal{U}_{P}, v \neq v_{1}} \zeta(1+v s)}{\prod_{v \in \mathcal{U}_{I}^{*}} \zeta(1+v s)} G_{r-1}(s) x^{v_{0} s},
$$

où $s$ satisfait $s_{r}=s_{r}^{*}$ et $G_{r-1}(s)$ désigne $G_{r}(s)$ pour ce choix $s_{r}=s_{r}^{*}$.

Nous pouvons maintenant dans une deuxième étape répéter le procédé avec chacune des intégrales $\int_{N_{r-1}(\kappa-i T)}^{N_{r-1}(\kappa+i T)}$ des résidus obtenus. Ou alors, dans le cas où certains coefficients $c_{r-1}, \ldots, c_{r-j}$ consécutifs décrits plus haut (pour un $j \geq 1$ ) sont nuls, répéter le procédé avec chacune des intégrales $\int_{N_{r-j-1}(\kappa-i T)}^{N_{r-j-1}(\kappa+i T)}$ par rapport à une nouvelle variable $s_{n_{r-1}}$ (qu'à partir de maintenant nous dénoterons tout de même par $s_{r-1}$ afin de ne pas alourdir une notation déjà assez pesante).

Remarque 6. La notation est à partir d'ici simplifiée, et on aura toujours à l'esprit la Remarque 4 : en fait, les choix possibles d'une succession 
de pôles $s_{n_{r}}^{*}, \ldots, s_{n_{r-l_{n}+1}}^{*}$ se feront, selon les termes de $I$ en cours d'évaluation, sur des permutations de l'ordre (choisi arbitrairement a priori) des variables $s_{r}, \ldots, s_{1}$, et le nombre de termes $l_{n}$ dans une telle succession n'est pas toujours le même. Ainsi, dans la suite de ce travail, il sera souvent approprié de penser $s_{n_{j}}$ et $l_{n}$ lorsqu'on lira $s_{j}$ et $l$. Cette dernière notation simplifiée sera en effet souvent utilisée par la suite (elle ne le sera cependant évidemment pas lorsqu'il sera nécessaire de considérer l'ensemble composé de la totalité des permutations des variables, comme au Paragraphe 2.9).

Remarque 7. Lorsque nous parlerons par la suite de la " $m+1$-ème étape" du procédé d'évaluation de $I$, nous entendrons par là que, pour une certaine succession de pôles $s_{n_{r}}^{*}, \ldots, s_{n_{r-l_{n}+1}^{*}}$, nous sommes sur le point d'appliquer une $m+1$-ème fois le procédé (a) décrit ci-dessus (à la variable $\left.s_{n_{r-m}}\right)$. Ceci sans tenir compte du nombre d'applications qu'il a été nécessaire de faire jusqu'ici du procédé (b), qui ne modifie pas la forme générale des termes à traiter par (a) (voir (18)).

Dans cette deuxième étape on ne peut plus garantir que chaque pôle, correspondant à un choix $s_{r-1}=s_{r-1}^{*}$, est nécessairement d'ordre 1 . Le résidu associé peut donc avoir une expression d'une forme plus compliquée que $R(1)$, et que nous décrirons plus précisément dans le Paragraphe 2.6. Disons simplement pour l'instant qu'apparaissent dans l'expression de ce résidu des dérivées, par rapport à la variable $s_{r-1}$, de chacun des facteurs de $R(1)$. Ainsi, issu de $x^{v_{0} s}$ apparait un facteur de la forme $x^{v_{0} s} P(\log x)$, où $P$ est un polynôme (et où bien sûr $s_{r}=s_{r}^{*}\left(s_{1}, \ldots, s_{r-1}\right)$ et $s_{r-1}=$ $\left.s_{r-1}^{*}\left(s_{1}, \ldots, s_{r-2}\right)\right)$, et issues de

$$
G_{r-1}(s)=\frac{H_{r-1}(s)}{\left(s_{r}^{*} \zeta\left(1+s_{r}^{*}\right)\right)^{-1} \prod_{i=1}^{r-1}\left(s_{i} \zeta\left(1+s_{i}\right)\right)^{-1}}
$$

apparaissent certaines dérivées $G_{r-1}^{(i)}(s)$ de cette fonction (avec les mêmes conditions sur $s$ ), dont nous baptiserons génériquement $G_{r-2}(s)$ chaque terme ("monomial"). De même nous baptiserons génériquement $H_{r-2}$ les dérivées $H_{r-1}^{(i)}$. Chacun des facteurs $P(\log x)$ et $G_{r-1}^{(i)}$ est sans singularité, de plus les $H_{r-1}^{(i)}$ sont d'un ordre de grandeur sans influence sur le résultat final (Lemme 9).

L'exposant $v_{0}$ du facteur $x^{v_{0} s}$ est d'une importance capitale, et nous suivrons son évolution jusqu'à la fin du procédé : chaque fois qu'il n'est pas annulé par la suite des transformations linéaires qu'on lui fait subir, la contribution du terme associé est négligeable.

Les seuls facteurs intervenant dans l'expression de ce nouveau résidu qui ont des singularités sont des dérivées d'un certain ordre des $\zeta(1+v s)$ 
du numérateur de $R(1)$ (par rapport à $s_{r-1}$ ), l'ordre du pôle associé étant susceptible de provenir de plusieurs de ces facteurs, ou encore d'être diminué voire annihilé par certains des facteurs du dénominateur.

Dans une troisième étape le procédé suivi n'est pas essentiellement différent de celui de la deuxième étape (quoique les calculs requis sont d'une nature plus complexe) : nous considérons séparément chacune des intégrales $\int_{N_{r-2}(\kappa-i T)}^{N_{r-2}(\kappa+i T)}$ (où, éventuellement, soit $N_{r-2}$ est remplacé par un autre nombre positif, soit encore, dans certains cas particuliers où le procédé (b) a été utilisé une ou plusieurs fois avant la deuxième étape, les bornes d'intégrations sont remplacées par des nombres de la forme $N_{j} \kappa \pm N_{2}^{-m} i T$. Nous remplaçons les résidus obtenus au pas précédent par les résidus de leurs pôles en certaines valeurs $s_{r-2}=s_{r-2}^{*}\left(s_{1}, \ldots, s_{r-3}\right)$. Ces derniers pôles étant un sous-ensemble de ceux de

$$
\prod_{v \in \mathcal{U}_{P}, v \neq v_{1}} \zeta(1+v s) / \prod_{v \in \mathcal{U}_{I}^{*}} \zeta(1+v s),
$$

et $s_{r-2}=s_{r-2}^{*}$ étant chaque fois une combinaison linéaire de $s_{1}, \ldots, s_{r-3}$, comme $s_{r-1}=s_{r-1}^{*}$ en est une de $s_{1}, \ldots, s_{r-2}$, et $s_{r}=s_{r}^{*}$ en est une de $s_{1}, \ldots, s_{r-2}$.

Dans des étapes successives nous poursuivons ce procédé tant qu'une application du cas (a) reste possible.

REMARQue 8. À ce sujet notons qu'il est bien sûr possible qu'une fois arrivés à une certaine étape $m$ du procédé d'évaluation nous ne disposions d'aucune singularité $s_{r-(m-1)}^{*}$ de la variable $s_{r-(m-1)}$, alors que d'autres singularités $s_{r-(M-1)}^{*}$ de certaines variables $s_{r-(M-1)}$ subsistent pour des étapes $M>m$ ultérieures. Il n'est pas nécessaire dans un tel cas de procéder à une nouvelle permutation de variables, puisqu'alors comme déjà mentionné il suit du Lemme 18 que la contribution du terme considéré est petite et peut donc être négligée.

Ainsi dorénavant, nous pourrons supposer avoir choisi une suite de "vrais" pôles $s_{r}=s_{r}^{*}, s_{r-1}=s_{r-1}^{*}, \ldots, s_{r-(l-1)}=s_{r-(l-1)}^{*}$ pour lesquels, après avoir calculé les résidus successifs associés, nous obtenons à la $l$-ème étape un dernier résidu, qui soit n'a plus aucune singularité, soit a un exposant de $x$ nul.

Avec une telle suite de choix et une telle convention, nous montrons (Lemme 14) que nous nous arrêtons nécessairement avant que la totalité des $r$ intégrales ait été effectuée, c'est-à-dire que l'on a toujours $l \leq r-1$. Nous montrons alors (Lemme 15) que, dans le cas où $r$ est impair, l'exposant $v_{0} s$ de $x$ n'a pas pu être annulé par la suite de substitutions $s_{r}^{*}, s_{r-1}^{*}, \ldots, s_{r-l-1}^{*}$. Il suit (Paragraphe 2.10) que la contribution correspondant à chaque suite de substitutions est négligeable lorsque $r$ est impair, d'où le (ii) du Théorème 1, 
et que lorsque la contribution correspondant à une certaine suite de substitutions n'est pas négligeable (dans le cas où $r$ est pair), cette contribution est de la forme $P(\log x)$ où $P$ est un polynôme, d'où le (i) du Théorème 1 . Dans le paragraphe suivant nous décrivons formellement le choix d'une suite de pôles.

2.4. Suites de pôles. Le choix d'une succession de pôles $s_{r}^{*}, \ldots, s_{r-l+1}^{*}$ $(l \leq r)$ correspond à faire un choix de vecteurs $v_{1}, \ldots, v_{l}$ de $\mathcal{U}_{P}$ et de transformations linéaires auxiliaires $T_{1}, \ldots, T_{l}: \mathbb{R}^{r} \rightarrow \mathbb{R}^{r}$ satisfaisant les conditions $\left(\mathrm{H}_{i}\right)$ et $\left(\mathrm{D}_{i}\right)(i \leq l)$ ci-dessous. On aura à l'esprit la Remarque 6 .

Soit $e_{j}$ le $j$-ème vecteur de la base canonique.

$\left(\mathrm{H}_{1}\right) v_{1}=\left[t_{0,1}, t_{0,2}, \ldots, t_{0, r}=1\right]$.

$\left(\mathrm{D}_{1}\right)$ L'application $T_{1}: \mathbb{R}^{r} \rightarrow \mathbb{R}^{r}$ est définie par $T_{1} v_{1}=\mathcal{O}$, i.e., $T_{1}$ est donnée par la matrice

$$
\left[\begin{array}{ccccc}
1 & 0 & \cdots & 0 & -t_{0,1} \\
0 & 1 & \cdots & 0 & -t_{0,2} \\
\cdots & \cdots & \cdots & \cdots & \cdots \\
0 & 0 & \cdots & 1 & -t_{0, r-1} \\
0 & 0 & \cdots & 0 & 0
\end{array}\right] .
$$

En particulier $T_{1} v=[\ldots, 0]$ quel que soit $v \in \mathbb{R}^{r}$. Le pôle correspondant à ce choix de $v_{1}$ est $s_{r}=s_{r}^{*}$ tel que $v_{1} s=0$.

$\left(\mathrm{H}_{2}\right) T_{1} v_{2}=\left[t_{1,1}, \ldots, t_{1, r-1} \neq 0,0\right]$.

$\left(\mathrm{D}_{2}\right)$ L'application $T_{2}: \mathbb{R}^{r} \rightarrow \mathbb{R}^{r}$ est définie par $T_{2} T_{1} v_{2}=\mathcal{O}$ et $T_{2} e_{r}=e_{r}$, i.e., $T_{2}$ est donnée par la matrice

$$
\left[\begin{array}{cccccc}
1 & 0 & \cdots & 0 & -t_{1,1} / t_{1, r-1} & 0 \\
0 & 1 & \cdots & 0 & -t_{1,2} / t_{1, r-1} & 0 \\
\cdots & \cdots & \cdots & \ldots & \ldots \ldots \ldots \cdots \cdots & \ldots \\
0 & 0 & \cdots & 1 & -t_{1, r-2} / t_{1, r-1} & 0 \\
0 & 0 & \cdots & 0 & 0 & 0 \\
0 & 0 & \cdots & 0 & 0 & 1
\end{array}\right] .
$$

En particulier $T_{2} T_{1} v=[\ldots, 0,0]$ quel que soit $v \in \mathbb{R}^{r}$. Le pôle correspondant à ce choix de $v_{1}, v_{2}$ est $s_{r-1}=s_{r-1}^{*}$ tel que $T_{1} v_{2} s=0$.

$\left(\mathrm{H}_{l}\right) T_{l-1} \cdots T_{1} v_{l}=\left[t_{l-1,1}, \ldots, t_{l-1, r-(l-1)} \neq 0,0, \ldots, 0\right]$.

$\left(\mathrm{D}_{l}\right)$ L'application $T_{l}: \mathbb{R}^{r} \rightarrow \mathbb{R}^{r}$ est définie par $T_{l} \cdots T_{1} v_{l}=\mathcal{O}$ et $T_{l} e_{i}$ $=e_{i}(i=r-l+2, \ldots, r)$, i.e., $T_{l}$ est donnée par 


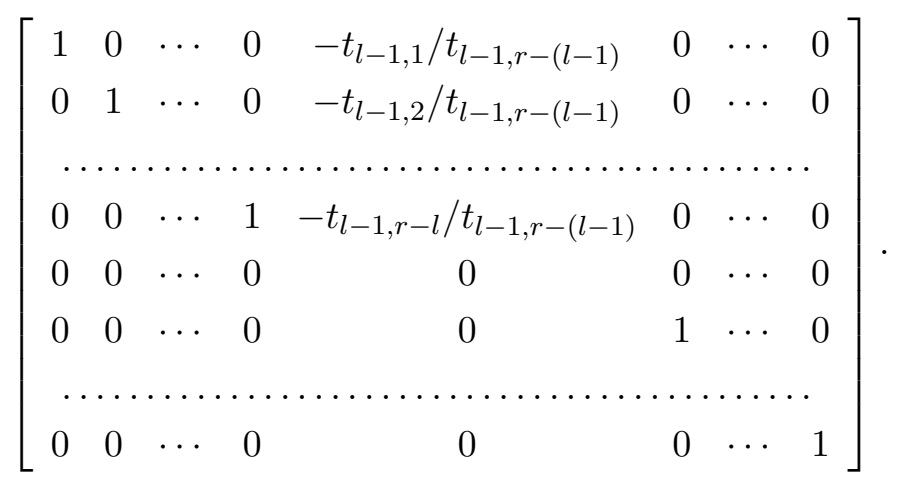

Le pôle correspondant à ce choix de $v_{1}, \ldots, v_{l}$ est $s_{r-l+1}=s_{r-l+1}^{*}$ tel que $T_{(l-1)} v_{l} s=0$.

Notons encore que dans le cas où $l=r-1$ nous avons

$\left(\mathrm{H}_{r-1}\right) T_{r-2} \cdots T_{1} v_{r-1}=\left[t_{r-2,1}, t_{r-2,2} \neq 0,0, \ldots, 0\right]$.

$\left(\mathrm{D}_{r-1}\right) T_{r-1}$ est définie par $T_{r-1} \cdots T_{1} v_{r-1}=\mathcal{O}$ et $T_{r-1} e_{i}=e_{i}(i \geq 3)$; sa matrice est donnée par

$$
\left[\begin{array}{ccccc}
1 & -t_{r-2,1} / t_{r-2,2} & 0 & \cdots & 0 \\
0 & 0 & 0 & \cdots & 0 \\
0 & 0 & 1 & \cdots & 0 \\
\ldots \ldots \ldots \ldots \ldots \ldots & \ldots \ldots & \ldots & \ldots \\
0 & 0 & 0 & \cdots & 1
\end{array}\right] .
$$

Et nous verrons (Lemme 14) que $l \leq r-1$.

2.4.1. Quelques résultats auxiliaires. Nous laissons au lecteur le soin de vérifier le lemme suivant.

Lemme 6. Soit $T_{(j)}:=T_{j} T_{j-1} \cdots T_{1}(j \leq l)$.

- Pour $i=1, \ldots, l$, Ker $T_{i}$ est de dimension 1 .

- Pour $j=1, \ldots, l, \operatorname{dim}\left(\operatorname{Im} T_{(j)}\right)=r-j$.

Considérons maintenant les $l$ ensembles de vecteurs de $\mathbb{R}^{r}$,

$$
W_{j}:=\left\{v \in \mathbb{R}^{r}: T_{(j-1)} v \neq \mathcal{O}, T_{(j)} v=\mathcal{O}\right\} \quad(j=1, \ldots, l)
$$

(où $T_{0}$ désigne l'identité), et les espaces vectoriels

$$
V_{j}:=\bigcup_{i \leq j} W_{i}=\left\{v \in \mathbb{R}^{r}: T_{(j)} v=\mathcal{O}\right\}=\operatorname{Ker} T_{(j)} \quad(j=1, \ldots, l) .
$$

Notons que $V_{j}$ est de dimension $j$.

LEMME 7. En particulier, si $v_{j_{1}}$ et $v_{j_{2}}$ sont dans $W_{j}$, alors il existe $c \in \mathbb{R}$ telle que $v_{j_{1}}+c v_{j_{2}} \in V_{j-1}$. 
Nous noterons encore

$$
W_{j P}:=\mathcal{U}_{P} \cap W_{j} \quad \text { et } \quad W_{j I}^{*}:=\mathcal{U}_{I}^{*} \cap W_{j} .
$$

2.5. Les facteurs sans pôle des résidus successifs. Nous décrivons ici plus précisément les fonctions $G_{i}$ et $H_{i}$ apparaissant en facteurs dans les résidus obtenus successivement au cours du procédé d'évaluation de l'intégrale $r$-uple $I$, et mentionnées au Paragraphe 2.3.3. On rappelle que l'intégrand de cette dernière est

$$
\frac{\prod_{v \in \mathcal{U}_{P}} \zeta(1+v s)}{\prod_{v \in \mathcal{U}_{I}^{*}} \zeta(1+v s)} G_{r}(s) x^{v_{0} s}=: \Pi(s) G_{r}(s) x^{v_{0} s}
$$

où, si l'on pose

$$
\eta(s)=\frac{1}{\zeta(1+s) s}
$$

la fonction $G_{r}(s)$ satisfait

$$
G_{r}(s)=H_{r}(s) \prod_{i=1}^{r} \eta\left(s_{i}\right)
$$

pour une fonction $H_{r}(s)$ régulière lorsque toutes les variables $s_{i}$ satisfont $\sigma_{i}>-1 /(2 r)$, et bornée lorsqu'elles satisfont $\sigma_{i} \geq-1 /(2 r+1)$.

C'est évidemment la fonction $\Pi(s)$ de (19) qui contient tous les pôles exploités pour évaluer $I$. Les facteurs qui en sont issus, et apparaissent dans l'expression des résidus successifs obtenus, seront décrits au Paragraphe 2.6. Ici nous nous contentons de décrire les facteurs issus de la fonction $G_{r}(s)$.

À la première étape, les pôles en $s_{r}=s_{r}^{*}\left(s_{r-1}, \ldots, s_{1}\right)$ sont tous d'ordre 1 , et aucune dérivée des facteurs de (19) n'intervient encore. Le facteur provenant de $G_{r}(s)$ dans l'expression du résidu, défini par $T_{1} v_{1}=\mathcal{O}$, en $s_{r}=s_{r}^{*}=$ $T_{1} s_{r}=\sum_{i \leq r-1} c_{i} s_{i}$, peut s'écrire sous la forme

$$
G_{r-1}(s)=H_{r-1}(s) \prod_{i=1}^{r-1} \eta\left(s_{i}\right) \cdot \eta\left(T_{1} s_{r}\right)
$$

où l'on note abusivement $T_{1} s_{r}$ pour $T_{1}\left[\begin{array}{c}0 \\ \vdots \\ 0 \\ s_{r}\end{array}\right]$, et où $G_{r-1}(s), H_{r-1}(s)$, sont $G_{r}(s), H_{r}(s)$, en $s_{r}=s_{r}^{*}=T_{1} s_{r}$.

À la deuxième étape, le facteur provenant de $G_{r-1}(s)$ dans l'expression du résidu, défini par $T_{2} T_{1} v_{2}=T_{(2)} v_{2}=\mathcal{O}$, en $s_{r-1}=s_{r-1}^{*}=T_{2} s_{r-1}$, peut s'écrire comme une somme de termes de la forme (à une constante multiplicative près) 


$$
\left(H_{r-1}(s)\right)^{(a)} \prod_{i=1}^{r-2} \eta\left(s_{i}\right) \cdot\left(\eta\left(s_{r-1}\right)\right)^{\left(a_{r-1,2}\right)}\left(\eta\left(T_{1} s_{r}\right)\right)^{\left(a_{r, 2}\right)},
$$

où l'écriture $\left(f\left(\sum c_{i} s_{i}\right)\right)^{(a)}, a \geq 0$, désigne ici la fonction obtenue en dérivant $a$ fois $f\left(\sum c_{i} s_{i}\right)$ par rapport à la variable $s_{r-1}$, puis en remplaçant celle-ci par $s_{r-1}^{*}=T_{2} s_{r-1}=T_{(2)} s_{r-1}$. Remarquons que lors de cette opération $T_{1} s_{r}$ est remplacé par $T_{(2)} s_{r}$, puisque

$$
T_{(2)} s_{r}=T_{2}\left(\sum_{i \leq r-1} c_{i} s_{i}\right)=\sum_{i \leq r-2} c_{i} s_{i}+c_{r-1} T_{2} s_{r-1} .
$$

Ainsi, nous avons $\left(\eta\left(s_{r-1}\right)\right)^{\left(a_{r-1,2}\right)}=\eta^{\left(a_{r-1,2}\right)}\left(T_{(2)} s_{r-1}\right)$, et $\left(\eta\left(T_{1} s_{r}\right)\right)^{\left(a_{r, 2}\right)}=$ $c_{r-1}^{a_{r, 2}} \eta^{\left(a_{r, 2}\right)}\left(T_{(2)} s_{r}\right)$. On notera génériquement $H_{r-2}(s)$ pour chaque facteur $\left(H_{r-1}(s)\right)^{(a)}$, et $G_{r-2}(s)$ pour chacun des termes de la forme (20), qui peut ainsi se récrire, à une constante multiplicative $c_{r-1}^{a_{r, 2}}$ près,

$$
G_{r-2}(s)=H_{r-2}(s) \prod_{i=1}^{r-2} \eta\left(s_{i}\right) \cdot \prod_{i=r-1}^{r} \eta^{\left(a_{i, 2}\right)}\left(T_{(2)} s_{i}\right) .
$$

En généralisant cet argument on obtient par récurrence le résultat suivant.

LEMME 8. À la m-ème étape le facteur, provenant de dérivées successives de $G_{r-1}(s)$, dans l'expression du résidu défini par $T_{(m)} v_{m}=\mathcal{O}$, en $s_{r-m+1}=s_{r-m+1}^{*}=T_{m} s_{r-m+1}$, peut s'écrire comme une somme de termes qui sont chacun, à une constante multiplicative près, de la forme

$$
G_{r-m}(s)=H_{r-m}(s) \prod_{i=1}^{r-m} \eta\left(s_{i}\right) \cdot \prod_{i=r-m+1}^{r} \eta^{\left(a_{i, m}\right)}\left(T_{(m)} s_{i}\right),
$$

où le facteur $H_{r-m}(s)$ est obtenu en dérivant successivement $H_{r}(s) b_{i} \geq 0$ fois par rapport à la variable $s_{r-i+1}(1 \leq i \leq m)$, puis en remplaçant chaque fois $s_{r-i+1}$ par $s_{r-i+1}^{*}=T_{i} s_{r-i+1}$.

D'autre part, les fonctions $H_{r-m}(s)$ se comportent bien.

LEMME 9. Il existe une constante $c>0$ telle que les fonctions $H_{r-m}(s)$ du Lemme 8 sont régulières et bornées lorsque toutes les variables $s_{i}$ satisfont $\left|\sigma_{i}\right| \leq c$.

Preuve. Par le Théorème 3, la série de Dirichlet pour $\widetilde{H}_{r}(s)$, mettons

$$
\widetilde{H}_{r}(s)=\sum_{n_{i} \geq 1} \frac{\left|h\left(n_{1}, \ldots, n_{r}\right)\right|}{n_{1}^{s_{1}} \cdots n_{r}^{s_{r}}}
$$

est donc convergente si $\sigma_{i}>-1 /(2 r)(1 \leq i \leq r)$, et de plus bornée si $\sigma_{i} \geq-1 /(2 r+1)(1 \leq i \leq r)$. Les dérivées successives de $H$ par rapport à certaines des variables $s_{i}$, qui produisent les fonctions $H_{r-m}$, font apparaître 
un nombre fini de séries de Dirichlet de la forme

$$
C \sum_{n_{i} \geq 1} \frac{h\left(n_{1}, \ldots, n_{r}\right)\left(\log n_{1}\right)^{M_{1}} \cdots\left(\log n_{r}\right)^{M_{r}}}{n_{1}^{s_{1}} \cdots n_{r-m}^{s_{r-m}} n_{r-m+1}^{s_{r-m+1}^{\prime}} \cdots n_{r}^{s_{r}^{\prime}}},
$$

où $C$ est une constante, les $M_{i}$ sont des entiers positifs, et où $s_{r-m+i}^{\prime}=$ $\sum_{j=1}^{r-m} c_{i, j} s_{j}$ pour des constantes $c_{i, j}$. Donc si $c>0$ est suffisamment petite, et si $\left|\sigma_{i}\right| \leq c(1 \leq i \leq r)$, toutes les séries de la forme (22) apparaissant dans l'expression de $H_{r-m}\left(s_{1}, \ldots, s_{r-m}\right)$ sont convergentes et bornées.

Il nous faut encore des estimations pour la fonction $1 / \zeta$ et pour les dérivées de la fonction $\zeta$. Pour énoncer le Théorème 1 nous avions introduit la notation $\delta(x)$. De façon similaire nous notons génériquement $\varepsilon(x)$ toute fonction $\exp \left(-C(\log x)^{2 / 5}\right)=: \varepsilon_{C}(x)$. De sorte que nous avons pour tout nombre réel $R_{\varepsilon}$ et tout nombre réel positif $R_{\delta}$,

$$
\delta(x)^{R_{\delta}} \varepsilon(x)^{R_{\varepsilon}} \leq \delta(x) .
$$

Lemme 10. Soit $M$ un entier positif ou nul, et $C$ une constante positive. Dans le domaine $\left\{\sigma=\Re s \geq 1-C /(\log T)^{3 / 5}, 1 \leq|t|=|\Im s| \leq T\right\}$, la dérivée $M$-ème de $\zeta(s)$ satisfait

$$
\zeta^{(M)}(s) \leq \varepsilon(T)^{-1} \leq \varepsilon(x)^{-1} .
$$

(Cette estimation est certainement très mauvaise ; mais elle suffit à nos besoins.)

Preuve. On sait que dans ce domaine, si $N$ est un entier positif (voir par exemple le Paragraphe 3.5 de [THB]),

$$
\zeta(s)-\sum_{n=1}^{N} \frac{1}{n^{s}}=s \int_{N}^{\infty} \frac{[u]-u+1 / 2}{u^{s+1}} d u+\frac{N^{1-s}}{s-1}-\frac{1}{2} N^{-s} .
$$

Si nous dérivons les deux côtés de cette égalité $M$ fois $(M \geq 0)$, nous obtenons

$$
\begin{aligned}
\zeta^{(M)}(s)+(-1)^{M+1} & \sum_{n=1}^{N} \frac{(\log n)^{M}}{n^{s}} \\
& \ll t \int_{N}^{\infty} \frac{(\log u)^{M}}{u^{\sigma+1}} d u+\frac{(\log N)^{M} N^{1-\sigma}}{t}+(\log N)^{M} N^{-\sigma} \\
& \ll \frac{t(\log N)^{M}}{N^{\sigma}}+\frac{N^{1-\sigma}(\log N)^{M}}{t}+(\log N)^{M} N^{-\sigma} .
\end{aligned}
$$

Posons maintenant $N=[t]$. La dernière majoration de $(24)$ est $\leq 1 / \varepsilon(T) \leq$ $1 / \varepsilon(x)$, et la somme apparaissant à gauche de (24) est également $\leq 1 / \varepsilon(x)$ puisque dans le domaine considéré $\left|n^{-s}\right| \leq 1 /(n \varepsilon(T))$. 
Lemme 11. Soit $M$ un entier positif ou nul. Il existe une constante positive A telle que dans le domaine $\left\{\sigma=\Re s \geq-A /(\log T)^{2 / 3}(\log \log T)^{1 / 3}\right.$, $|t|=|\Im s| \leq T\}$, la dérivée $M$-ème de $\eta$ satisfait

$$
\eta^{(M)}(s)=\frac{A_{M}(s)}{s+1} \quad \text { avec } \quad\left|A_{M}(s)\right| \leq 1 / \varepsilon(x) .
$$

Preuve. Cette estimation suit du Lemme 10, de l'estimation connue, dans le domaine considéré ici,

$$
1 / \zeta(1+s) \ll(\log T)^{2 / 3}(\log \log T)^{1 / 3}
$$

(voir par exemple le Paragraphe 6.19 de [THB]), et de la représentation $\left.s \zeta(s+1)=1+\gamma s+O\left(s^{2}\right)\right)$ valable dans un voisinage de $s=0$.

Les Lemmes 8, 9 et 11 livrent également l'estimation suivante, à laquelle nous ferons appel dans les preuves des Lemmes 19 et 20 .

Lemme 12. Soient $s_{j}=\sigma_{j}+i t_{j}(j=1, \ldots, r)$, et supposons que pour une constante positive $C,\left|t_{j}\right| \leq C T(j=1, \ldots, r)$. Alors il existe une constante $a>0$ absolue telle que, dans le domaine $\left\{\left|\sigma_{j}\right| \leq a /(\log T)^{2 / 3}(\log \log T)^{1 / 3}\right.$, $j=1, \ldots, r\}$, toutes les fonctions $G_{r-m}(m=0,1, \ldots, l)$ du Lemme 8 satisfont, pour chaque $j_{0}=r-m+1, \ldots, r$,

$$
\left|G_{r-m}(s)\right| \leq \frac{1}{\varepsilon(x)} \prod_{i=1}^{r-m} \frac{1}{\left|t_{i}\right|+1} \cdot \frac{1}{\left|T_{(m)} t_{j_{0}}\right|+1} .
$$

2.6. Les trois premiers pas du procédé d'évaluation. Comme décrit dans le Paragraphe 2.3, nous évaluons l'intégrale multiple en commençant par l'intégrale la plus intérieure (par rapport à la variable $s_{r}$ ), puis en remplaçant à chaque étape l'intégrale intérieure d'une somme de fonctions obtenues à l'étape précédente par une somme de résidus. Ceci tant que cette opération n'est pas soit triviale soit inutile, c'est-à-dire tant qu'un pôle du dernier intégrand obtenu est "disponible", et tant que l'exposant de $x$ n'a pas été annulé. Ainsi nous remplaçons l'intégrale multiple de départ, $I$, par une somme d'intégrales (simples ou multiples, mais d'une multiplicité inférieure à $r$ ), dont l'intégrand (i) n'a plus aucune singularité, ou alors (ii) a un exposant nul en $x$. Chacune de ces intégrales est obtenue par un choix de pôles successifs $s_{r}=s_{r}^{*}\left(s_{1}, \ldots, s_{r-1}\right), s_{r-1}=s_{r-1}^{*}\left(s_{1}, \ldots, s_{r-2}\right), \ldots, s_{r-l+1}=$ $s_{r-l+1}^{*}\left(s_{1}, \ldots, s_{r-l}\right)$. Comme indiqué, nous montrons qu'en tous les cas $l \leq$ $r-1$, soit que $r-l+1 \geq 2$ (Lemme 14). Comme nous l'avons vu au Paragraphe 2.4, chaque tel choix successif de pôles correspond à faire un choix de vecteurs $v_{1}, \ldots, v_{l}$ de $\mathcal{U}_{P}$ et à définir les applications linéaires $T_{j}$ et $T_{(j)}$ $(j=1, \ldots, l)$ (avec lesquelles la condition (ii) peut se récrire $T_{(l)} v_{0} s=0$ ).

Ça n'est qu'à partir de la troisième étape que l'on voit clairement comment décrire une étape quelconque du processus, et nous commençons donc 
par donner une description exhaustive des trois premiers pas. Ceci nous permet d'autre part d'introduire peu à peu les notations requises.

Terminologie. Afin de simplifier et d'uniformiser certaines notations et définitions plus bas, nous dirons qu'une fonction a un pôle d'ordre 0 en $t$ si elle est entière et non nulle en ce point, et d'ordre $-n$ pour un entier $n$ positif si elle a un zéro d'ordre $n$ en $t$.

Premier pas. Il est clair que $W_{1 P}=\left\{v_{1}\right\}$ et que $W_{1 I}^{*}=\emptyset$. Le pôle correspondant $s_{r}^{*}$ tel que $v_{1} s=0$, est donc d'ordre 1 , et le résidu correspondant est

$$
\prod_{v \in \mathcal{U}_{P} \backslash\left\{v_{1}\right\}} \zeta\left(1+T_{1} v s\right) \prod_{v \in \mathcal{U}_{I}^{*}} \zeta\left(1+T_{1} v s\right)^{-1} G_{r-1}\left(s_{1}, \ldots, s_{r-1}\right) x^{T_{1} v_{0} s},
$$

où $G_{r-1}\left(s_{1}, \ldots, s_{r-1}\right)$ désigne $G_{r}(s)$ en $s_{r}=s_{r}^{*}$.

Convention de notation. Par le Lemme 9, dans toute la suite des opérations le facteur $H_{r-1}\left(s_{1}, \ldots, s_{r-1}\right)$ de $G_{r-1}\left(s_{1}, \ldots, s_{r-1}\right)$ n'aura pas d'influence sur l'ordre de grandeur du résultat final. Pour simplifier la notation nous ignorerons dans ce paragraphe les facteurs provenant de $G_{r-1}$, ainsi que les éventuelles constantes apparaissant en facteur lors des calculs de résidus, sans oublier toutefois que ces facteurs existent : nous ferons usage des Lemmes 8, 11 et 12 dans la preuve du théorème.

On aura d'autre part toujours à l'esprit la Remarque 6. Avec cette convention le premier résidu s'écrit

$$
R(1)=\tau_{1}=\tau_{10}=\prod_{v \in \mathcal{U}_{P} \backslash V_{1}} \zeta\left(1+T_{1} v s\right) \prod_{v \in \mathcal{U}_{I}^{*} \backslash V_{1}} \zeta\left(1+T_{1} v s\right)^{-1} x^{T_{1} v_{0} s} .
$$

(La justification du choix des symboles $\tau_{1}$ et $\tau_{10}$ apparaîtra dans les étapes suivantes.) Nous écrivons, pour chaque $v \in \mathcal{U}_{P} \backslash V_{1}$,

$$
\zeta\left(1+T_{1} v s\right)=: \zeta(P, 1, v, s)
$$

et pour chaque $v \in \mathcal{U}_{I}^{*} \backslash V_{1}$,

$$
\zeta\left(1+T_{1} v s\right)^{-1}=: \zeta(I, 1, v, s)
$$

(L'explication du choix de cette notation apparaîtra également dans la suite.) Nous définissons maintenant les ordres de ces fonctions,

$$
\mathcal{O}(\zeta(P, 1, v, s))=1, \quad \mathcal{O}(\zeta(I, 1, v, s))=-1
$$

(qui correspondent à l'ordre des pôles de ces fonctions en $T_{1} v s=0$ ), puis l'ordre total du seul terme $\tau_{1}=\tau_{10}$ de $R(1)$,

$$
\begin{aligned}
\mathcal{O}_{1}\left(\tau_{1}\right) & =\mathcal{O}(R(1))=\sum_{v \in \mathcal{U}_{P} \backslash V_{1}} \mathcal{O}(\zeta(P, 1, v, s))+\sum_{v \in \mathcal{U}_{I}^{*} \backslash V_{1}} \mathcal{O}(\zeta(I, 1, v, s)) \\
& =\left|\mathcal{U}_{P} \backslash V_{1}\right|-\left|\mathcal{U}_{I}^{*} \backslash V_{1}\right|=\left(2^{r-1}-2\right)-\left(2^{r-1}-r\right)=r-2 .
\end{aligned}
$$


Notons que l'ordre total $\mathcal{O}(R(1))$ est une borne inférieure à la somme des ordres de tous les pôles de $R(1)$.

Deuxième pas. Commençons par définir l'ordre en $v_{2}$ de $\tau_{1}\left(=\tau_{10}=\right.$ $R(1))$,

$$
\mathcal{O}_{v_{2}}\left(\tau_{1}\right)=\mathcal{O}_{v_{2}}\left(\tau_{10}\right)=\mathcal{O}_{v_{2}}(R(1)):=\left|W_{2 P}\right|-\left|W_{2 I}^{*}\right|,
$$

que nous appelons $B_{2}$. $B_{2}$ est l'ordre du pôle en $s_{r-1}^{*}$ : en effet, si $v \in W_{2 P}$ $\cup W_{2 I}^{*}$, alors par le Lemme $7, T_{1} v=c T_{1} v_{2}$, et donc si $T_{1} v_{2} s=0$, alors $T_{1} v s=0$ aussi.

Remarque 9. Nous aurions pu noter, avant le premier pas,

$$
\begin{aligned}
R(0) & :=\prod_{v \in \mathcal{U}_{P}} \zeta(1+v s) \prod_{v \in \mathcal{U}_{I}^{*}} \zeta(1+v s)^{-1} x^{v_{0} s} \\
& =\prod_{v \in \mathcal{U}_{P}} \zeta(P, 0, v, s) \prod_{v \in \mathcal{U}_{I}^{*}} \zeta(I, 0, v, s) x^{v_{0} s}=: \tau_{0}=\tau_{00},
\end{aligned}
$$

puis

$$
\mathcal{O}_{v_{1}}\left(\tau_{0}\right)=\mathcal{O}_{v_{1}}\left(\tau_{00}\right)=\mathcal{O}_{v_{1}}(R(0)):=\left|W_{1 P}\right|-\left|W_{1 I}^{*}\right|=1=: B_{1} .
$$

Notons que $B_{1}=1$ est l'ordre du pôle en $s_{r}^{*}$, que

$$
B_{2}=\left|W_{2 P}\right|-\left|W_{2 I}^{*}\right|=B_{1}-1+\left|W_{2 P}\right|-\left|W_{2 I}^{*}\right|,
$$

et que l'ordre total de $\tau_{0}$ peut être défini par

$$
\mathcal{O}_{0}\left(\tau_{0}\right)=\mathcal{O}(R(0)):=\left|\mathcal{U}_{P}\right|-\left|\mathcal{U}_{I}^{*}\right|=r-1 .
$$

Les facteurs $\zeta\left(1+T_{1} v s\right)$ correspondant aux $v \in W_{2 P} \cup W_{2 I}^{*}$ disparaissent simultanément, lors du calcul du résidu en $s_{r-1}^{*}\left(s_{1}, \ldots, s_{r-2}\right)$ tel que $T_{1} v_{2} s$ $=0$. Comme l'ordre de ce pôle est $B_{2}$, l'expression du résidu en $s_{r-1}^{*}$ fait intervenir les dérivées d'ordres $0,1, \ldots, B_{2}-1$ du facteur résiduel

$$
\begin{aligned}
F R\left(\tau_{1}\right) & =\prod_{v \in \mathcal{U}_{P} \backslash V_{2}} \zeta\left(1+T_{1} v s\right) \prod_{v \in \mathcal{U}_{I}^{*} \backslash V_{2}} \zeta\left(1+T_{1} v s\right)^{-1} x^{T_{1} v_{0} s} \\
& =\prod_{v \in \mathcal{U}_{P} \backslash V_{2}} \zeta(P, 1, v, s) \prod_{v \in \mathcal{U}_{I}^{*} \backslash V_{2}} \zeta(I, 1, v, s) x^{T_{1} v_{0} s} .
\end{aligned}
$$

Cette fois, si $B_{2}>1$, le résidu $R(2)$ comprendra plusieurs termes $\tau_{2}$ de la forme

$$
\tau_{2}=\prod_{v \in \mathcal{U}_{P} \backslash V_{2}} \zeta\left(P, 2, v, i_{2}, s\right) \prod_{v \in \mathcal{U}_{I}^{*} \backslash V_{2}} \zeta\left(I, 2, v, j_{2}, s\right) x^{T_{(2)} v_{0} s} P_{2}(\log x),
$$

où $P_{2}$ est un certain polynôme (dépendant de $\tau_{2}$ ), où $\zeta\left(P, 2, v, i_{2}, s\right)$ est la $i_{2}$-ème dérivée de $\zeta(P, 1, v, s)$ par rapport à $s_{r-1}$ en $s$ tel que $s_{r}=s_{r}^{*}$ et $s_{r-1}=s_{r-1}^{*}$, où de même $\zeta\left(I, 2, v, j_{2}, s\right)$ est la $j_{2}$-ème dérivée de $\zeta(I, 1, v, s)$, 
et où $i_{2}=i_{2}\left(v, \tau_{2}\right), j_{2}=j_{2}\left(v, \tau_{2}\right)$. De plus, pour chaque $\tau_{2}$ on a

$$
\sum_{v \in \mathcal{U}_{P} \backslash V_{2}} i_{2}+\sum_{v \in \mathcal{U}_{I}^{*} \backslash V_{2}} j_{2} \leq B_{2}-1
$$

À ce stade on peut encore aisément écrire $\zeta\left(P, 2, v, i_{2}, s\right)$ explicitement à l'aide de la fonction $\zeta$ de Riemann : c'est $\pm \zeta^{\left(i_{2}\right)}\left(1+T_{(2)} v s\right)$, qui en $T_{(2)} v s=0$ a un pôle d'ordre $i_{2}+1$. Quant à $\zeta\left(I, 2, v, j_{2}, s\right)$, c'est $\left(\zeta^{-1}\right)^{\left(j_{2}\right)}\left(1+T_{(2)} v s\right)$, qui a un zéro d'ordre 1 (soit un pôle d'ordre -1 avec notre convention) en $T_{(2)} v s=0$ si $j_{2}=0$, et qui est entière (soit a un pôle d'ordre $\leq 0$ en $\left.T_{(2)} v s=0\right)$ si $j_{2}>0$. Similairement à (26) nous définissons donc les ordres de ces fonctions qui vérifient

$$
\mathcal{O}\left(\zeta\left(P, 2, v, i_{2}, s\right)\right)=i_{2}+1
$$

et

$$
\mathcal{O}\left(\zeta\left(I, 2, v, j_{2}, s\right)\right) \leq \overline{\mathcal{O}}\left(\zeta\left(I, 2, v, j_{2}, s\right)\right):= \begin{cases}-1 & \text { si } j_{2}=0, \\ 0 & \text { si } j_{2}>0,\end{cases}
$$

puis comme en (27) l'ordre total de $\tau_{2}$,

$$
\mathcal{O}_{2}\left(\tau_{2}\right)=\sum_{v \in \mathcal{U}_{P} \backslash V_{2}} \mathcal{O}\left(\zeta\left(P, 2, v, i_{2}, s\right)\right)+\sum_{v \in \mathcal{U}_{I}^{*} \backslash V_{2}} \mathcal{O}\left(\zeta\left(I, 2, v, j_{2}, s\right)\right),
$$

ainsi que son majorant $\overline{\mathcal{O}}_{2}\left(\tau_{2}\right)$, obtenu en remplaçant les $\mathcal{O}$ par les $\overline{\mathcal{O}}$ correspondants dans la deuxième somme de (34). Notons qu'on a

$$
\begin{aligned}
\mathcal{O}_{2}\left(\tau_{2}\right) & \leq \overline{\mathcal{O}}_{2}\left(\tau_{2}\right)=\sum_{v \in \mathcal{U}_{P} \backslash V_{2}}\left(i_{2}+1\right)-\sum_{\substack{v \in \mathcal{U}_{I}^{*} \backslash V_{2} \\
j_{2}=0}} 1 \\
& =\sum_{v \in \mathcal{U}_{P} \backslash V_{2}} i_{2}+\sum_{v \in \mathcal{U}_{I}^{*} \backslash V_{2}} j_{2}+\left|\mathcal{U}_{P} \backslash V_{2}\right|-\sum_{v \in \mathcal{U}_{I}^{*} \backslash V_{2}} \max \left(j_{2}, 1\right) \\
& \leq B_{2}-1+\left|\mathcal{U}_{P} \backslash V_{2}\right|-\sum_{v \in \mathcal{U}_{I}^{*} \backslash V_{2}} \max \left(j_{2}, 1\right) \\
& =\mathcal{O}_{1}\left(\tau_{1}\right)-1+\left|\mathcal{U}_{I}^{*} \backslash V_{2}\right|-\sum_{v \in \mathcal{U}_{I}^{*} \backslash V_{2}} \max \left(j_{2}, 1\right) \leq \mathcal{O}_{1}\left(\tau_{1}\right)-1 \\
& \leq B_{2}-1+\left|\mathcal{U}_{P} \backslash V_{2}\right|-\left|\mathcal{U}_{I}^{*} \backslash V_{2}\right|,
\end{aligned}
$$

où nous avons fait appel à (27) et (30) pour obtenir la dernière égalité et la dernière majoration. Nous définissons maintenant l'ordre total de $R(2)$ par

$$
\mathcal{O}(R(2)):=\mathcal{O}_{2}\left(\tau_{20}\right)
$$

où

$$
\begin{aligned}
& \tau_{20}:= \\
& \zeta\left(P, 2, v_{3}, B_{2}-1, s\right) \prod_{v \in \mathcal{U}_{P} \backslash\left(V_{2} \cup\left\{v_{3}\right\}\right)} \zeta(P, 2, v, 0, s) \prod_{v \in \mathcal{U}_{I}^{*} \backslash V_{2}} \zeta(I, 2, v, 0, s) x^{T_{(2)} v_{0} s} .
\end{aligned}
$$


Cette définition est appropriée, puisque pour tout $\tau_{2}$ on a

$$
\mathcal{O}_{2}\left(\tau_{2}\right) \leq \overline{\mathcal{O}}_{2}\left(\tau_{2}\right) \leq \mathcal{O}_{2}\left(\tau_{20}\right)=\mathcal{O}(R(2))
$$

En effet,

$$
\mathcal{O}_{2}\left(\tau_{20}\right)=B_{2}-1+\left|\mathcal{U}_{P} \backslash V_{2}\right|-\left|\mathcal{U}_{I}^{*} \backslash V_{2}\right| .
$$

Troisième pas. Si $v \in W_{3 P} \cup W_{3 I}^{*}$, alors $T_{(2)} v=c T_{(2)} v_{3}$, et donc si $T_{(2)} v_{3} s=0$, alors $T_{(2)} v s=0$ aussi. Ainsi, similairement à l'étape précédente les facteurs d'un $\tau_{2}$ correspondant aux $v \in W_{3 P} \cup W_{3 I}^{*}$ disparaissent simultanément, lors du calcul du résidu du pôle en $s_{r-2}^{*}$ tel que $T_{(2)} v_{3} s=0$.

Comme au pas précédent, nous commençons par définir $\mathcal{O}_{v_{3}}\left(\tau_{2}\right)$, l'ordre de $v_{3}$ en $\tau_{2}$, qui est l'ordre du pôle de $\tau_{2}$ en $T_{(2)} v_{3} s=0$,

$$
\mathcal{O}_{v_{3}}\left(\tau_{2}\right)=\sum_{v \in W_{3 P}} \mathcal{O}\left(\zeta\left(P, 2, v, i_{2}, s\right)\right)+\sum_{v \in W_{3 I}^{*}} \mathcal{O}\left(\zeta\left(I, 2, v, j_{2}, s\right)\right) .
$$

Similairement à (35) notons que

$$
\begin{aligned}
\mathcal{O}_{v_{3}}\left(\tau_{2}\right) & \leq \sum_{v \in W_{3 P}}\left(i_{2}+1\right)-\sum_{\substack{v \in W_{3 I}^{*} \\
j_{2}=0}} 1 \\
& =\sum_{v \in W_{3 P}} i_{2}+\sum_{v \in W_{3 I}^{*}} j_{2}+\left|W_{3 P}\right|-\sum_{v \in W_{3 I}^{*}} \max \left(j_{2}, 1\right) \\
& \leq B_{2}-1+\left|W_{3 P}\right|-\left|W_{3 I}^{*}\right| .
\end{aligned}
$$

Et notons aussi que

$$
B_{3}:=B_{2}-1+\left|W_{3 P}\right|-\left|W_{3 I}^{*}\right|=\mathcal{O}_{v_{3}}\left(\tau_{20}\right),
$$

ce qui implique en particulier que $B_{3}$ majore l'ordre du pôle $B_{3}^{\prime}$ en $s_{k-2}^{*}$.

Remarque 10. A-t-on forcément $B_{3}=B_{3}^{\prime}$ ? Ça n'est pas évident, et cette incertitude ne simplifie pas la notation. Il faut donc distinguer entre l'ordre $B_{m}^{\prime}$ du pôle de la fonction somme de tous les termes $\tau_{m-1}$ (au $m$-ème pas), et l'ordre maximal $B_{m}$ atteint par le pôle d'un certain $\tau_{m-1}: \tau_{m-1,0}$ est bien sûr un tel $\tau_{m-1}$, mais est-ce le seul? On gardera d'autre part à l'esprit la Remarque 6 .

Le facteur résiduel de $\tau_{2}$ qui apparaît dérivé de 0 à au plus $B_{3}-1$ fois dans le calcul du résidu en $s_{r-2}^{*}$ est

$$
F R\left(\tau_{2}\right)=\prod_{v \in \mathcal{U}_{P} \backslash V_{3}} \zeta\left(P, 2, v, i_{2}, s\right) \prod_{v \in \mathcal{U}_{I}^{*} \backslash V_{3}} \zeta\left(I, 2, v, j_{2}, s\right) x^{T_{(2)} v_{0} s} P_{2}(\log x),
$$

et le résidu $R(3)$ comprend donc, pour chaque $\tau_{2}$, des termes $\tau_{3}=\tau_{3}\left(\tau_{2}\right)$ qui s'écrivent

$$
\tau_{3}=\prod_{v \in \mathcal{U}_{P} \backslash V_{3}} \zeta\left(P, 3, v, i_{2}, i_{3}, s\right) \prod_{v \in \mathcal{U}_{I}^{*} \backslash V_{3}} \zeta\left(I, 3, v, j_{2}, j_{3}, s\right) x^{T_{(3)} v_{0} s} P_{3}(\log x),
$$


où $P_{3}=P_{3}\left(\tau_{3}\right)$ est un polynôme, où $i_{3}=i_{3}\left(v, \tau_{2}\right)$ et où $\zeta\left(P, 3, v, i_{2}, i_{3}, s\right)=$ $\zeta^{\left(i_{3}\right)}\left(P, 2, v, i_{2}, s\right)$, le suffixe $\left(i_{3}\right)$ signifiant que la fonction est dérivée $i_{3}$ fois par rapport à la variable $s_{r-2}$, en $s_{r-2}=s_{r-2}^{*}$ (de même pour $j_{3}$ et $\zeta(I, \ldots)$ ). Comme $\mathcal{O}_{v_{3}}\left(\tau_{2}\right)$ est l'ordre du pôle de $\tau_{2}$ en $T_{(2)} v_{3} s=0$, nous avons de plus

$$
\sum_{v \in \mathcal{U}_{P} \backslash V_{3}} i_{3}+\sum_{v \in \mathcal{U}_{I}^{*} \backslash V_{3}} j_{3} \leq \mathcal{O}_{v_{3}}\left(\tau_{2}\right)-1 \leq B_{3}-1
$$

Chaque fonction $\zeta(P, 3, \ldots)$ est la dérivée $i_{3}$-ème d'une fonction ayant en $T_{(3)} v s=0$ un pôle d'ordre $i_{2}+1$, et a donc en ce point un pôle d'ordre $i_{2}+i_{3}+1$. Chaque fonction $\zeta(I, 3, \ldots)$ est la dérivée $j_{3}$-ème d'une fonction ayant en ce même point un pôle d'ordre -1 si $j_{2}=0$ et d'ordre non positif dans tous les cas; c'est donc une fonction ayant en ce point un pôle d'ordre -1 si $j_{2}+j_{3}=0$ et d'ordre non positif dans tous les cas. Les ordres de ces fonctions en $T_{(3)}$ vs $=0$ vérifient ainsi

$$
\mathcal{O}\left(\zeta\left(P, 3, v, i_{2}, i_{3}, s\right)\right)=i_{2}+i_{3}+1
$$

et

$$
\begin{aligned}
\mathcal{O}\left(\zeta\left(I, 3, v, j_{2}, j_{3}, s\right)\right) & \leq \overline{\mathcal{O}}\left(\zeta\left(I, 3, v, j_{2}, j_{3}, s\right)\right) \\
& := \begin{cases}-1 & \text { si } j_{2}+j_{3}=0 \\
0 & \text { si } j_{2}+j_{3}>0\end{cases}
\end{aligned}
$$

et nous pouvons définir l'ordre total de $\tau_{3}$,

$$
\mathcal{O}_{3}\left(\tau_{3}\right)=\sum_{v \in \mathcal{U}_{P} \backslash V_{3}} \mathcal{O}\left(\zeta\left(P, 3, v, i_{2}, i_{3}, s\right)\right)+\sum_{v \in \mathcal{U}_{I}^{*} \backslash V_{3}} \mathcal{O}\left(\zeta\left(I, 3, v, j_{2}, j_{3}, s\right)\right),
$$

ainsi que son majorant $\overline{\mathcal{O}}_{3}\left(\tau_{3}\right)$ obtenu en remplaçant les $\mathcal{O}$ par les $\overline{\mathcal{O}}$ correspondants dans la deuxième somme de (42). Nous avons

$$
\begin{aligned}
\mathcal{O}_{3}\left(\tau_{3}\right) \leq \overline{\mathcal{O}}_{3}\left(\tau_{3}\right)=\sum_{v \in \mathcal{U}_{P} \backslash V_{3}}\left(i_{2}+i_{3}+1\right)-\sum_{\substack{v \in \mathcal{U}_{I}^{*} \backslash V_{3} \\
j_{2}+j_{3}=0}} 1 \\
=\sum_{v \in \mathcal{U}_{P} \backslash V_{3}}\left(i_{2}+i_{3}\right)+\sum_{v \in \mathcal{U}_{I}^{*} \backslash V_{3}}\left(j_{2}+j_{3}\right)+\left|\mathcal{U}_{P} \backslash V_{3}\right|-\sum_{v \in \mathcal{U}_{I}^{*} \backslash V_{3}} \max \left(j_{2}+j_{3}, 1\right) \\
=\sum_{v \in \mathcal{U}_{P} \backslash V_{2}} i_{2}+\sum_{v \in \mathcal{U}_{I}^{*} \backslash V_{2}} j_{2}-\left(\sum_{v \in W_{3 P}} i_{2}+\sum_{v \in W_{3 I}^{*}} j_{2}\right) \\
\quad+\sum_{v \in \mathcal{U}_{P} \backslash V_{3}} i_{3}+\sum_{v \in \mathcal{U}_{I}^{*} \backslash V_{3}} j_{3}+\left|\mathcal{U}_{P} \backslash V_{3}\right|-\sum_{v \in \mathcal{U}_{I}^{*} \backslash V_{3}} \max \left(j_{2}+j_{3}, 1\right)
\end{aligned}
$$




$$
\begin{aligned}
\leq & \overline{\mathcal{O}}_{2}\left(\tau_{2}\right)-\left|\mathcal{U}_{P} \backslash V_{2}\right|+\sum_{v \in \mathcal{U}_{I}^{*} \backslash V_{2}} \max \left(j_{2}, 1\right) \\
& -\mathcal{O}_{v_{3}}\left(\tau_{2}\right)+\left|W_{3 P}\right|-\sum_{v \in W_{3}^{*}} \max \left(j_{2}, 1\right) \\
& +\mathcal{O}_{v_{3}}\left(\tau_{2}\right)-1+\left|\mathcal{U}_{P} \backslash V_{3}\right|-\sum_{v \in \mathcal{U}_{I}^{*} \backslash V_{3}} \max \left(j_{2}+j_{3}, 1\right) \\
\leq & \overline{\mathcal{O}}_{2}\left(\tau_{2}\right)-1+\sum_{v \in \mathcal{U}_{I}^{*} \backslash V_{3}} \max \left(j_{2}, 1\right)-\sum_{v \in \mathcal{U}_{I}^{*} \backslash V_{3}} \max \left(j_{2}+j_{3}, 1\right) \\
\leq & \overline{\mathcal{O}}_{2}\left(\tau_{2}\right)-1,
\end{aligned}
$$

où nous avons fait appel à (35), (38), (40.) De plus, par (43), (35) et (39) nous avons

$$
\begin{aligned}
\mathcal{O}_{3}\left(\tau_{3}\right) & \leq \overline{\mathcal{O}}_{3}\left(\tau_{3}\right) \\
& \leq \overline{\mathcal{O}}_{2}\left(\tau_{2}\right)-1+\sum_{v \in \mathcal{U}_{I}^{*} \backslash V_{3}} \max \left(j_{2}, 1\right)-\sum_{v \in \mathcal{U}_{I}^{*} \backslash V_{3}} \max \left(j_{2}+j_{3}, 1\right) \\
& \leq B_{2}-2+\left|\mathcal{U}_{P} \backslash V_{2}\right|-\sum_{v \in W_{3 I}^{*}} \max \left(j_{2}, 1\right)-\sum_{v \in \mathcal{U}_{I}^{*} \backslash V_{3}} \max \left(j_{2}+j_{3}, 1\right) \\
& \leq B_{3}-1+\left|\mathcal{U}_{P} \backslash V_{3}\right|-\sum_{v \in \mathcal{U}_{I}^{*} \backslash V_{3}} \max \left(j_{2}+j_{3}, 1\right)
\end{aligned}
$$

d'où

$$
\mathcal{O}_{3}\left(\tau_{3}\right) \leq B_{3}-1+\left|\mathcal{U}_{P} \backslash V_{3}\right|-\left|\mathcal{U}_{I}^{*} \backslash V_{3}\right|=\mathcal{O}_{3}\left(\tau_{30}\right)
$$

où

$$
\begin{aligned}
\tau_{30}:= & \zeta\left(P, 3, v_{4}, 0, B_{3}-1, s\right) \\
& \times \prod_{v \in \mathcal{U}_{P} \backslash\left(V_{3} \cup\left\{v_{4}\right\}\right)} \zeta(P, 3, v, 0,0, s) \prod_{v \in \mathcal{U}_{I}^{*} \backslash V_{3}} \zeta(I, 3, v, 0,0, s) x^{T_{(3)} v_{0} s} .
\end{aligned}
$$

Similairement à l'étape précédente nous pouvons donc définir l'ordre total de $R(3)$ par

$$
\mathcal{O}(R(3)):=\mathcal{O}_{3}\left(\tau_{30}\right)
$$

Nous sommes maintenant prêts à décrire un argument de récurrence qui nous permet de passer des étapes $1, \ldots, m-1$ à la $m$-ème étape.

2.7. Le $m$-ème pas. Si $v \in W_{m P} \cup W_{m I}^{*}$, alors $T_{(m-1)} v=c T_{(m-1)} v_{m}$, et donc si $T_{(m-1)} v_{m} s=0$, alors $T_{(m-1)} v s=0$ aussi. Donc les facteurs d'un $\tau_{m-1}$ correspondant aux $v \in W_{m P} \cup W_{m I}^{*}$ disparaissent simultanément, lors du calcul du résidu du pôle en $s_{r-(m-1)}^{*}$ tel que $T_{(m-1)} v_{m} s=0$. Ici $\tau_{m-1}$ est un des termes du résidu $R(m-1)$ obtenu à la $m-1$-ème étape, avec 
$\tau_{m-1}=\tau_{m-1}\left(\tau_{m-2}\right), \tau_{m-2}=\tau_{m-2}\left(\tau_{m-3}\right), \ldots, \tau_{3}=\tau_{3}\left(\tau_{2}\right)$ et $\tau_{2}=\tau_{2}\left(\tau_{1}\right)=$ $\tau_{2}\left(\tau_{10}\right)=\tau_{2}(R(1)) ; \tau_{m-1}$ a une expression de la forme

$$
\begin{aligned}
\tau_{m-1}= & \prod_{v \in \mathcal{U}_{P} \backslash V_{m-1}} \zeta\left(P, m-1, v, i_{2}, \ldots, i_{m-1}, s\right) \\
& \times \prod_{v \in \mathcal{U}_{I}^{*} \backslash V_{m-1}} \zeta\left(I, m-1, v, j_{2}, \ldots, j_{m-1}, s\right) x^{T_{(m-1)} v_{0} s} P_{m-1}(\log x),
\end{aligned}
$$

où $i_{t}=i_{t}\left(v, \tau_{t-1}\right)$. Les ordres de chacun des facteurs de $\tau_{m-1}$ ont été considérés au pas précédent; ce sont les ordres des pôles de ces facteurs, qui vérifient

$$
\mathcal{O}\left(\zeta\left(P, m-1, v, i_{2}, \ldots, i_{m-1}, s\right)\right)=i_{2}+\cdots+i_{m-1}+1
$$

et

$$
\mathcal{O}\left(\zeta\left(I, m-1, v, j_{2}, \ldots, j_{m-1}, s\right)\right) \leq \overline{\mathcal{O}}\left(\zeta\left(I, m-1, v, j_{2}, \ldots, j_{m-1}, s\right)\right)
$$

où

$$
\overline{\mathcal{O}}\left(\zeta\left(I, m-1, v, j_{2}, \ldots, j_{m-1}, s\right)\right)= \begin{cases}-1 & \text { si } j_{2}+\cdots+j_{m-1}=0 \\ 0 & \text { si } j_{2}+\cdots+j_{m-1}>0\end{cases}
$$

Nous définissons maintenant $\mathcal{O}_{v_{m}}\left(\tau_{m-1}\right)$, l'ordre de $v_{m}$ en $\tau_{m-1}$, qui est l'ordre du pôle de $\tau_{m-1}$ en $T_{(m-1)} v_{m} s=0$,

$$
\begin{aligned}
\mathcal{O}_{v_{m}}\left(\tau_{m-1}\right)= & \sum_{v \in W_{m P}} \mathcal{O}\left(\zeta\left(P, m-1, v, i_{2}, \ldots, i_{m-1}, s\right)\right) \\
& +\sum_{v \in W_{m I}^{*}} \mathcal{O}\left(\zeta\left(I, m-1, v, j_{2}, \ldots, j_{m-1}, s\right)\right) .
\end{aligned}
$$

Nous avons

$$
\begin{aligned}
\mathcal{O}_{v_{m}}\left(\tau_{m-1}\right) \leq & \sum_{v \in W_{m P}}\left(i_{2}+\cdots+i_{m-1}+1\right)-\sum_{\substack{v \in W_{m I}^{*} \\
j_{2}+\cdots+j_{m-1}=0}} 1 \\
= & \sum_{v \in W_{m P}}\left(i_{2}+\cdots+i_{m-1}\right)+\sum_{v \in W_{m I}^{*}}\left(j_{2}+\cdots+j_{m-1}\right) \\
& +\left|W_{m P}\right|-\sum_{v \in W_{m I}^{*}} \max \left(j_{2}+\cdots+j_{m-1}, 1\right) .
\end{aligned}
$$

Nous montrerons au paragraphe suivant que, pour tout $\tau_{m-1}$,

$$
\mathcal{O}_{v_{m}}\left(\tau_{m-1}\right) \leq \mathcal{O}_{v_{m}}\left(\tau_{m-1,0}\right)=B_{m}
$$


où

$$
\begin{aligned}
\tau_{m-1,0}:= & \zeta\left(P, m-1, v_{m}, 0, \ldots, 0, B_{m-1}-1, s\right) \\
& \times \prod_{v \in \mathcal{U}_{P} \backslash\left(V_{m-1} \cup\left\{v_{m}\right\}\right)} \zeta(P, m-1, v, 0, \ldots, 0, s) \\
& \times \prod_{v \in \mathcal{U}_{I}^{*} \backslash V_{m-1}} \zeta(I, m-1, v, 0, \ldots, 0, s) z^{T_{(m-1)} v_{0} s},
\end{aligned}
$$

et où $B_{m}$ est défini par récurrence par

$$
B_{m}=B_{m-1}-1+\left|W_{m, P}\right|-\left|W_{m, I}^{*}\right| .
$$

Remarque 11. Notons que par conséquent $B_{m}$ majore l'ordre $B_{m}^{\prime}$ (voir la Remarque 10) du pôle en $s_{r-(m-1)}^{*}$.

Le facteur résiduel de $\tau_{m-1}$ qui apparaît dérivé de 0 à au plus $B_{m}-1$ fois dans le calcul du résidu en $s_{r-(m-1)}^{*}$ est

$$
\begin{aligned}
F R\left(\tau_{m-1}\right)= & \prod_{v \in \mathcal{U}_{P} \backslash V_{m}} \zeta\left(P, m-1, v, i_{2}, \ldots, i_{m-1}, s\right) \\
& \times \prod_{v \in \mathcal{U}_{I}^{*} \backslash V_{m}} \zeta\left(I, m-1, v, j_{2}, \ldots, j_{m-1}, s\right) x^{T_{(m-1)} v_{0} s} P_{m-1}(\log x),
\end{aligned}
$$

et ce résidu qu'on note $R(m)$ comprend donc, pour chaque $\tau_{m-1}$, des termes $\tau_{m}=\tau_{m}\left(\tau_{m-1}\right)$ qui s'écrivent

$$
\begin{aligned}
\tau_{m}= & \prod_{v \in \mathcal{U}_{P} \backslash V_{m}} \zeta\left(P, m, v, i_{2}, \ldots, i_{m}, s\right) \\
& \times \prod_{v \in \mathcal{U}_{I}^{*} \backslash V_{m}} \zeta\left(I, m, v, j_{2}, \ldots, j_{m}, s\right) x^{T_{(m)} v_{0} s} P_{m}(\log x),
\end{aligned}
$$

où $i_{m}=i_{m}\left(v, \tau_{m-1}\right)$, et où $\zeta\left(P, m, v, i_{2}, \ldots, i_{m}, s\right)=\zeta^{\left(i_{m}\right)}\left(P, m-1, v, i_{2}, \ldots\right.$, $\left.i_{m-1}, s\right)$, le suffixe $\left(i_{m}\right)$ signifiant que la fonction est dérivée $i_{m}$ fois par rapport à la variable $s_{r-(m-1)}$, en $s_{r-(m-1)}=s_{r-(m-1)}^{*}$ (de même pour $j_{m}$ et $\zeta(I, \ldots))$. Nous avons de plus, par $(50)$,

$$
\sum_{v \in \mathcal{U}_{P} \backslash V_{m}} i_{m}+\sum_{v \in \mathcal{U}_{I}^{*} \backslash V_{m}} j_{m} \leq \mathcal{O}_{v_{m}}\left(\tau_{m-1}\right)-1 \leq B_{m}-1 .
$$

Chaque fonction $\zeta(P, m, \ldots)$ est la dérivée $i_{m}$-ème d'une fonction ayant en $T_{(m)} v s=0$ un pôle d'ordre $i_{2}+\cdots+i_{m-1}+1$, et a donc en ce point un pôle d'ordre $i_{2}+\cdots+i_{m}+1$. Quant aux fonctions $\zeta(I, m, \ldots)$, ce sont des dérivées $j_{m}$-èmes d'une fonction ayant en ce même point un pôle d'ordre -1 si $j_{2}+\cdots+j_{m-1}=0$ et d'ordre non positif dans tous les cas ; c'est donc une fonction ayant en ce point un pôle d'ordre -1 si $j_{2}+\cdots+j_{m}=0$ et d'ordre non positif dans tous les cas. Les ordres de ces fonctions en $T_{(m)} v s=0$ 
satisfont ainsi

$$
\begin{aligned}
& \mathcal{O}\left(\zeta\left(P, m, v, i_{2}, \ldots, i_{m}, s\right)\right)=i_{2}+\cdots+i_{m}+1, \\
& \mathcal{O}\left(\zeta\left(I, m, v, j_{2}, \ldots, j_{m}, s\right)\right) \leq \overline{\mathcal{O}}\left(\zeta\left(I, m, v, j_{2}, \ldots, j_{m}, s\right)\right),
\end{aligned}
$$

où

$$
\overline{\mathcal{O}}\left(\zeta\left(I, m, v, j_{2}, \ldots, j_{m}, s\right)\right)= \begin{cases}-1 & \text { si } j_{2}+\cdots+j_{m}=0 \\ 0 & \text { si } j_{2}+\cdots+j_{m}>0\end{cases}
$$

et nous pouvons définir l'ordre total de $\tau_{m}$,

$$
\begin{aligned}
\mathcal{O}_{m}\left(\tau_{m}\right)= & \sum_{v \in \mathcal{U}_{P} \backslash V_{m}} \mathcal{O}\left(\zeta\left(P, m, v, i_{2}, \ldots, i_{m}, s\right)\right) \\
& +\sum_{v \in \mathcal{U}_{I}^{*} \backslash V_{m}} \mathcal{O}\left(\zeta\left(I, m, v, j_{2}, \ldots, j_{m}, s\right)\right),
\end{aligned}
$$

ainsi que son majorant $\overline{\mathcal{O}}_{m}\left(\tau_{m}\right)$ obtenu en remplaçant les $\mathcal{O}$ par les $\overline{\mathcal{O}}$ correspondants dans la deuxième somme de (54). Nous montrerons ci-dessous par récurrence que

$$
\overline{\mathcal{O}}_{m}\left(\tau_{m}\right) \leq \overline{\mathcal{O}}_{m-1}\left(\tau_{m-1}\right)-1
$$

et plus précisément que

$$
\begin{aligned}
\overline{\mathcal{O}}_{m}\left(\tau_{m}\right) \leq & \overline{\mathcal{O}}_{m-1}\left(\tau_{m-1}\right)-1+\sum_{v \in \mathcal{U}_{I}^{*} \backslash V_{m}} \max \left(j_{2}+\cdots+j_{m-1}, 1\right) \\
& -\sum_{v \in \mathcal{U}_{I}^{*} \backslash V_{m}} \max \left(j_{2}+\cdots+j_{m}, 1\right) \\
\leq & B_{m}-1+\left|\mathcal{U}_{P} \backslash V_{m}\right|-\sum_{v \in \mathcal{U}_{I}^{*} \backslash V_{m}} \max \left(j_{2}+\cdots+j_{m}, 1\right) .
\end{aligned}
$$

Nous avons donc

$$
\mathcal{O}_{m}\left(\tau_{m}\right) \leq \overline{\mathcal{O}}_{m}\left(\tau_{m}\right) \leq B_{m}-1+\left|\mathcal{U}_{P} \backslash V_{m}\right|-\left|\mathcal{U}_{I}^{*} \backslash V_{m}\right|=\mathcal{O}_{m}\left(\tau_{m, 0}\right)
$$

où

$$
\begin{aligned}
\tau_{m, 0}:= & \zeta\left(P, m, v_{m+1}, 0, \ldots, 0, B_{m}-1, s\right) \\
& \times \prod_{v \in \mathcal{U}_{P} \backslash\left(V_{m} \cup\left\{v_{m+1}\right\}\right)} \zeta(P, m, v, 0, \ldots, 0, s) \\
& \times \prod_{v \in \mathcal{U}_{I}^{*} \backslash V_{m}} \zeta(I, m, v, 0, \ldots, 0, s) x^{T_{(m)} v_{0} s} .
\end{aligned}
$$

Finalement, nous définissons l'ordre total de $R(m)$ par

$$
\mathcal{O}(R(m)):=\mathcal{O}_{m}\left(\tau_{m, 0}\right)
$$


2.7.1. Conclusion de l'argument : preuves de (50), (55) et (56). Nous démontrons que les inégalités (50), (55) et (56) sont satisfaites à chaque étape $m$, jusqu'à la fin du procédé d'évaluation. Notons que pour $m=2$ l'inégalité (50) est donnée par (28), et que pour $m=3$ elle est donnée par (38) (avec (39)). Quant aux inégalités (55) et (56), elles sont données par (35) pour $m=2$ et par (43) et (44) pour $m=3$. Montrons d'abord (50). Par (49) nous avons

$$
\begin{aligned}
& \mathcal{O}_{v_{m}}\left(\tau_{m-1}\right) \\
& \leq\left|W_{m P}\right|-\left|W_{m I}^{*}\right|+\sum_{v \in W_{m P}}\left(i_{2}+\cdots+i_{m-1}\right)+\sum_{v \in W_{m I}^{*}}\left(j_{2}+\cdots+j_{m-1}\right) \\
& \leq\left|W_{m P}\right|-\left|W_{m I}^{*}\right|+\sum_{v \in \mathcal{U}_{P} \backslash V_{m-1}} i_{m-1}+\sum_{v \in \mathcal{U}_{I}^{*} \backslash V_{m-1}} j_{m-1} \\
&+\sum_{v \in \mathcal{U}_{P} \backslash V_{m-2}} i_{m-2}+\sum_{v \in \mathcal{U}_{I}^{*} \backslash V_{m-2}} j_{m-2}+\cdots+\sum_{v \in \mathcal{U}_{P} \backslash V_{2}} i_{2}+\sum_{v \in \mathcal{U}_{I}^{*} \backslash V_{2}} j_{2} \\
& \quad-\sum_{v \in W_{m-1, P}}\left(i_{2}+\cdots+i_{m-2}\right)-\sum_{v \in W_{m-1, I}^{*}}\left(j_{2}+\cdots+j_{m-2}\right) \\
&-\sum_{v \in W_{m-2, P}}\left(i_{2}+\cdots+i_{m-3}\right)-\sum_{v \in W_{m-2, I}^{*}}\left(j_{2}+\cdots+j_{m-3}\right) \\
&-\cdots-\sum_{v \in W_{3 P}} i_{2}-\sum_{v \in W_{3 I}^{*}} j_{2} .
\end{aligned}
$$

À l'aide des relations (52) et (49), cette fois appliquées à $m-1, m-2, \ldots, 2$ dans la dernière estimation, à (28), et à la définition $B_{k}=B_{k-1}-1+\left|W_{k P}\right|-$ $\left|W_{k I}^{*}\right|, B_{1}=1$, nous obtenons donc

$$
\begin{aligned}
& \mathcal{O}_{v_{m}}\left(\tau_{m-1}\right) \\
& \leq\left|W_{m P}\right|-\left|W_{m I}^{*}\right|+\left(\mathcal{O}_{v_{m-1}}\left(\tau_{m-2}\right)-1\right)+\left(\mathcal{O}_{v_{m-2}}\left(\tau_{m-3}\right)-1\right)+\cdots \\
&+\left(\mathcal{O}_{v_{2}}\left(\tau_{1}\right)-1\right)-\mathcal{O}_{v_{m-1}}\left(\tau_{m-2}\right)+\left|W_{m-1, P}\right|-\left|W_{m-1, I}^{*}\right|-\mathcal{O}_{v_{m-2}}\left(\tau_{m-3}\right) \\
&+\left|W_{m-2, P}\right|-\left|W_{m-2, I}^{*}\right|+\cdots-\mathcal{O}_{v_{3}}\left(\tau_{2}\right)+\left|W_{3, P}\right|-\left|W_{3, I}^{*}\right| \\
&= B_{2}-(m-2)+\left|W_{3, P}\right|-\left|W_{3, I}^{*}\right|+\left|W_{4, P}\right|-\left|W_{4, I}^{*}\right| \\
&+\cdots+\left|W_{m, P}\right|-\left|W_{m, I}^{*}\right| \\
&= B_{3}-(m-3)+\left|W_{4, P}\right|-\left|W_{4, I}^{*}\right|+\left|W_{5, P}\right|-\left|W_{5, I}^{*}\right| \\
&+\cdots+\left|W_{m, P}\right|-\left|W_{m, I}^{*}\right| \\
& \vdots \\
&= B_{m-1}-1+\left|W_{m, P}\right|-\left|W_{m, I}^{*}\right|=B_{m},
\end{aligned}
$$


ce qui démontre (50). Passons aux inégalités (55) et (56). Nous avons

$$
\begin{aligned}
& \overline{\mathcal{O}}_{m}\left(\tau_{m}\right) \\
& =\sum_{v \in \mathcal{U}_{P} \backslash V_{m}}\left(i_{2}+\cdots+i_{m}+1\right)-\sum_{\substack{v \in \mathcal{U}_{I}^{*} \backslash V_{m} \\
j_{2}+\cdots+j_{m}=0}} 1 \\
& =\sum_{v \in \mathcal{U}_{P} \backslash V_{m}}\left(i_{2}+\cdots+i_{m}\right)+\sum_{v \in \mathcal{U}_{I}^{*} \backslash V_{m}}\left(j_{2}+\cdots+j_{m}\right) \\
& +\left|\mathcal{U}_{P} \backslash V_{m}\right|-\sum_{v \in \mathcal{U}_{I}^{*} \backslash V_{m}} \max \left(j_{2}+\cdots+j_{m}, 1\right) \\
& =\sum_{v \in \mathcal{U}_{P} \backslash V_{m}} i_{m}+\sum_{v \in \mathcal{U}_{I}^{*} \backslash V_{m}} j_{m} \\
& +\sum_{v \in \mathcal{U}_{P} \backslash V_{m-1}}\left(i_{2}+\cdots+i_{m-1}\right)+\sum_{v \in \mathcal{U}_{I}^{*} \backslash V_{m-1}}\left(j_{2}+\cdots+j_{m-1}\right) \\
& -\sum_{v \in W_{m P}}\left(i_{2}+\cdots+i_{m-1}\right)-\sum_{v \in W_{m I}^{*}}\left(j_{2}+\cdots+j_{m-1}\right) \\
& +\left|\mathcal{U}_{P} \backslash V_{m}\right|-\sum_{v \in \mathcal{U}_{I}^{*} \backslash V_{m}} \max \left(j_{2}+\cdots+j_{m}, 1\right) \\
& \leq \mathcal{O}_{v_{m}}\left(\tau_{m-1}\right)-1 \\
& +\overline{\mathcal{O}}_{m-1}\left(\tau_{m-1}\right)-\left|\mathcal{U}_{P} \backslash V_{m-1}\right|+\sum_{v \in \mathcal{U}_{I}^{*} \backslash V_{m-1}} \max \left(j_{2}+\cdots+j_{m-1}, 1\right) \\
& -\mathcal{O}_{v_{m}}\left(\tau_{m-1}\right)+\left|W_{m P}\right|-\sum_{v \in W_{m I}^{*}} \max \left(j_{2}+\cdots+j_{m-1}, 1\right) \\
& +\left|\mathcal{U}_{P} \backslash V_{m}\right|-\sum_{v \in \mathcal{U}_{I}^{*} \backslash V_{m}} \max \left(j_{2}+\cdots+j_{m}, 1\right) \\
& \leq \overline{\mathcal{O}}_{m-1}\left(\tau_{m-1}\right)-1 \\
& -\sum_{v \in \mathcal{U}_{I}^{*} \backslash V_{m}}\left(\max \left(j_{2}+\cdots+j_{m}, 1\right)-\max \left(j_{2}+\cdots+j_{m-1}, 1\right)\right) \\
& \leq \overline{\mathcal{O}}_{m-1}\left(\tau_{m-1}\right)-1,
\end{aligned}
$$

où nous avons utilisé (52), (60) pour $m-1$, et (49). Les inégalités (62) et (61) livrent (55) et la première inégalité de (56). Nous obtenons maintenant la deuxième inégalité de (56) à partir de (61) en lui appliquant (56) pour $m-1$. 


\subsection{Autres résultats auxiliaires}

LEMME 13. Si $1 \leq m \leq l$ alors

$$
B_{m}=1-m+\left|\mathcal{U}_{P} \cap V_{m}\right|-\left|\mathcal{U}_{I}^{*} \cap V_{m}\right| .
$$

Preuve. Par définition nous avons

$$
\begin{aligned}
B_{m} & =B_{m-1}-1+\left|W_{m P}\right|-\left|W_{m I}^{*}\right| \\
& =B_{m-2}-2+\left(\left|W_{m P}\right|+\left|W_{m-1, P}\right|\right)-\left(\left|W_{m I}^{*}\right|+\left|W_{m-1, I}^{*}\right|\right) \\
& \vdots \\
& =B_{1}-(m-1)+\sum_{2 \leq i \leq m}\left|W_{i P}\right|-\sum_{2 \leq i \leq m}\left|W_{i I}^{*}\right| \\
& =1-m+\left|\mathcal{U}_{P} \cap V_{m}\right|-\left|\mathcal{U}_{I}^{*} \cap V_{m}\right|,
\end{aligned}
$$

$\operatorname{car} B_{1}=1,\left|W_{1 P}\right|=1$ et $\left|W_{1 I}^{*}\right|=0$.

Lemme 14. Pour l comme dans la Remarque 8 on a toujours $l \leq r-1$.

Preuve. Supposons au contraire que $l=r$. Comme nous l'avions noté dans le Paragraphe 2.4.1, l'espace $V_{m}$ est de dimension $m$; par conséquent, $\mathcal{U}_{P} \cap V_{r}=\mathcal{U}_{P}$ et $\mathcal{U}_{I}^{*} \cap V_{r}=\mathcal{U}_{I}^{*}$. Par le Lemme 13 nous avons donc

$$
B_{r}=1-r+\left|\mathcal{U}_{P}\right|-\left|\mathcal{U}_{I}^{*}\right|=1-r+\left(2^{r-1}-1\right)-\left(2^{r-1}-r\right)=0 .
$$

Mais nous avons vu au paragraphe précédent (voir la Remarque 11) que $B_{r}$ majore l'ordre $B_{r}^{\prime}$ du pôle en $s_{1}^{*}$, qui n'est par conséquent pas un "vrai" pôle.

Les Lemmes 13 et 14 sont bien entendu valables pour tout entier positif $r$; le Lemme 15 qui suit permettra de démontrer le (ii) du Théorème 1.

LEMME 15. Si $r$ est impair, alors $v_{0} \notin V_{l}$.

Preuve. Si au contraire $v_{0} \in V_{l}$, alors pour chaque vecteur $v \in \mathcal{U}_{P} \cup\{\mathcal{O}\}$ nous avons

$$
v \in\left(\mathcal{U}_{P} \cap V_{l}\right) \cup\{\mathcal{O}\} \Leftrightarrow v_{0}-v \in \mathcal{U}_{I} \cap V_{l} .
$$

En d'autres termes,

$$
\left|\mathcal{U}_{P} \cap V_{l}\right|=\left|\mathcal{U}_{I} \cap V_{l}\right|-1 .
$$

D'autre part, comme $V_{l}$ est de dimension $l$, il ne peut contenir plus de $l$ vecteurs minimaux de $\mathcal{U}_{I}$, d'où

$$
\left|\mathcal{U}_{I} \cap V_{l}\right|-\left|\mathcal{U}_{I}^{*} \cap V_{l}\right| \leq l .
$$

Par le Lemme 13 nous avons donc

$$
\begin{aligned}
B_{l} & =1-l+\left|\mathcal{U}_{P} \cap V_{l}\right|-\left|\mathcal{U}_{I}^{*} \cap V_{l}\right| \\
& \leq 1-l+\left(\left|\mathcal{U}_{I} \cap V_{l}\right|-1\right)+\left(l-\left|\mathcal{U}_{I} \cap V_{l}\right|\right)=0,
\end{aligned}
$$


ce qui n'est pas possible : $B_{l}$ majore en effet l'ordre $B_{l}^{\prime}$ du pôle en $s_{r-(l-1)}^{*}$, qui par hypothèse est positif.

2.9. Choix des abscisses d'intégration. Rappelons tout d'abord que $\kappa=(\log x)^{-1}$ et $\log T=C(\log x)^{3 / 5}(\log \log x)^{-1 / 5}$ pour une constante positive $C$, et définissons $\beta:=(\log T)^{-2 / 3}(\log \log T)^{-1 / 3}$ (il suit donc que $T=x^{C(3 / 5)^{1 / 3} \beta(1+o(1))}$ et que $\beta=\left(5 /\left(3 C^{2}\right)\right)^{1 / 3}(\log x)^{-2 / 5}(\log \log x)^{-1 / 5}(1+$ $o(1)))$.

Dans ce paragraphe nous vérifions que les $N_{j}$ peuvent être choisis de façon à éviter qu'à la $m+1$-ème étape du processus d'évaluation décrit plus haut les chemins d'intégration des intégrales non encore évaluées nous fassent passer trop près d'un pôle de l'intégrand (voir le Paragraphe 2.3.2). Plus précisément, il faut éviter d'avoir un pôle proche de trois types de segments de droites — mettons à une distance $\leq C \kappa=C(\log x)^{-1}-$ (i) tout d'abord du segment vertical de l'intégrale en cours d'évaluation, qui est d'abscisse $N_{r-m} \kappa$ si aucune permutation des variables n'a encore été nécessaire, et sinon $N_{r-j} \kappa$ : pour un certain $j>m$ si l'on n'est pas encore parvenu à une variable permutée, pour un $j<m$ si l'on est parvenu à une telle variable); (ii) ensuite, des deux segments horizontaux exploités pour déformer ce chemin d'intégration vertical (d'ordonnées $\pm N_{r-j} T$ pour un certain $j \geq m$ si l'on n'est pas encore parvenu à une variable permutée, et d'ordonnées réduites de façon adéquate sinon) ; (iii) et finalement du segment vertical complétant le rectangle, dont l'abscisse sera de la forme $\pm b \beta$ pour une constante $b>0$ dont nous préciserons les propriétés au Paragraphe 2.10 (voir le Lemme 17).

Comme il est ici sans objet de chercher à minimaliser la taille des $N_{j}$, puisqu'ils sont de toute façon en nombre fini, donc bornés, ce choix des $N_{j}$ est effectué plus facilement si pour les définir nous faisons intervenir, non pas seulement les suites de pôles qui sont effectivement utilisées dans le processus d'évaluation, mais toutes les suites de pôles possibles. Nous procédons ainsi. Tout d'abord, pour chaque permutation $P=\left(s_{n_{r}}, s_{n_{r-1}}, \ldots, s_{n_{1}}\right)$ de l'ordre d'intégration, nous remplaçons (formellement) successivement chaque intégrale intérieure par une somme de résidus (voir la Remarque 6) ; à la $M+1$ ème étape nous obtenons un intégrand ayant des pôles en certains $s_{n_{r-M}}=$ $s_{n_{r-M}}^{*}=s_{n_{r-1}}^{*}\left(s_{n_{1}}, \ldots, s_{n_{r-M-1}}\right)$ satisfaisant une équation linéaire de la forme $f_{r-M}=0$ si $s_{n_{r-M}}=s_{n_{r-M}}^{*}$, où $f_{r-M}:=c_{n_{1}} s_{n_{1}}+\cdots+c_{n_{r-M-1}} s_{n_{r-M-1}}$ $+c_{n_{r-M}} s_{n_{r-M}}$ (et où certains des coefficients $c_{n_{j}}$ peuvent bien sûr être nuls).

Maintenant, nous appelons $L$ l'ensemble comprenant la totalité des formes linéaires $f_{j}(j \geq 1)$ apparaissant lorsqu'on applique ce processus formel successivement à chacune des permutations $P$ de l'ordre d'intégration. Pour $J$ entier positif nous définissons $L_{J}$ comme le sous-ensemble de $L$ con- 
tenant toutes les formes linéaires qui peuvent se récrire sous la forme $g_{J}=$ $c_{1} s_{1}+\cdots+c_{J} s_{J}$, avec $c_{J} \neq 0$. (Notons que, dans cette écriture, $J$ n'est pas forcément le $n_{r-M}$ d'un $f_{r-M}$.) Puis soit $C_{-}$la plus petite valeur absolue d'un coefficient quelconque $c_{j} \neq 0$ apparaissant dans une forme linéaire de $L$, $C_{+}$la plus grande, et $K:=2 C_{+} / C_{-}$. Enfin nous définissons

$$
N_{J}:=K^{J-1} \quad(J \geq 1) .
$$

On montre facilement par récurrence sur $J$ que

$$
N_{J} \geq \max _{g_{J} \in L_{J}} \frac{\left|c_{1}\right|+\left|c_{2}\right| N_{2}+\cdots+\left|c_{J-1}\right| N_{J-1}}{\left|c_{J}\right|}+1 .
$$

Lemme 16. Si les nombres $N_{J}$ sont définis en (63) (et satisfont donc (64)), alors les déformations rectangulaires que l'on fait subir aux chemins d'intégration lors du procédé d'évaluation de I décrit au Paragraphe 2.3 passent toutes à une distance $\geq\left(C_{-} / C_{+}\right) \kappa$ de tout pôle de l'intégrand.

Preuve. Il faut s'assurer que les propriétés (i)-(iii) ci-dessus sont vérifiées pour $C=C_{-} / C_{+}$.

(i) Dans un processus formel de remplacement successif des intégrales intérieures, selon une permutation quelconque $P=\left(s_{n_{r}}, s_{n_{r-1}}, \ldots, s_{n_{1}}\right)$ de l'ordre d'intégration, par des sommes de résidus, nous voulons nous assurer que le segment d'intégration par rapport à la variable $s_{n_{r-m}}$ ne contient pas de $s$ avec $\left|s-s_{n_{r-m}}^{*}\right|<C \kappa$ pour un pôle $s_{n_{r-m}}=s_{n_{r-m}}^{*}$ avec $f_{r-m}:=$ $c_{n_{1}} s_{n_{1}}+\cdots+c_{n_{r-m-1}} s_{n_{r-m-1}}+c_{n_{r-m}} s_{n_{r-m}}=0$ en $s_{n_{r-m}}^{*}$, soit pas de $s$ avec

$$
\left|c_{n_{1}} s_{n_{1}}+\cdots+c_{n_{r-m-1}} s_{n_{r-m-1}}+c_{n_{r-m}} s\right|<\left|c_{n_{r-m}}\right| C \kappa,
$$

ce que l'on peut faire en considérant la partie réelle $\left(c_{n_{1}} N_{n_{1}}+\cdots+\right.$ $\left.c_{n_{r-m-1}} N_{n_{r-m-1}}+c_{n_{r-m}} N_{n_{r-m}}\right) \kappa$ de la combinaison linéaire à gauche de (65). En effet, comme la forme linéaire $f_{r-m}$ appartient à un $L_{J}$ pour un certain entier $J \geq 1$, cette partie réelle peut se récrire $\left(c_{1} N_{1}+\cdots+c_{J} N_{J}\right) \kappa$ pour des coefficients $c_{j}$ avec $c_{J} \neq 0$, et donc le fait que $\left|c_{1} N_{1}+\cdots+c_{J} N_{J}\right| \kappa \geq\left|c_{J}\right| \kappa \geq$ $C_{-} \kappa \geq\left|c_{n_{r-m}}\right| C \kappa$ est une conséquence immédiate de la propriété (64). Ceci montre que (65) n'est effectivement pas possible, donc que (i) est satisfait.

(iii) Comme nous avons $\kappa \lll \beta$, le point (iii) est évident (pour $x$ assez grand).

(ii) Pour vérifier le point (ii) on remarque d'abord que par (63) quel que soit le nombre $j$ de fois que la procédure (b) a été utilisée lorsqu'on se prépare à suivre pour la $m+1$-ème fois la procédure (a), les ordonnées des extrémités des segments verticaux d'intégration des intégrales restantes ont été réduites et sont (dans l'ordre de droite à gauche) de la forme $\pm K^{r-m-j-1} i T, \ldots$, $\pm K i T, \pm i T, \pm K^{-1} i T, \pm K^{-j-1} i T$, soit, pour $\tau:=K^{-j-1} T$, de la forme $\pm N_{m} i \tau, \ldots, \pm i \tau$. Le point (ii) suit maintenant de (64), comme (i), en considérant cette fois la partie imaginaire de la combinaison linéaire à gauche 
de (65) sur les segments horizontaux. (Et en fait la distance entre un pôle et un segment horizontal est d'ordre au moins $T$.)

2.10. Démonstration du Théorème 1. Avec tout ce qui précède nous voyons que, comme nous l'avions annoncé au Paragraphe 2.3.2, lorsque nous nous préparons à effectuer la $m+1$-ème étape du procédé d'évaluation de $I$ (voir la Remarque 7), nous nous trouvons en présence de termes qui sont de la forme

$$
\begin{aligned}
& P_{m}(\log x) \\
& \times \int_{N_{n_{1}} \kappa-i K^{-q} T}^{N_{n_{1}} \kappa+i K^{-q} T} \frac{1}{\zeta\left(1+s_{n_{1}}\right) s_{n_{1}}} \cdots \int_{N_{n_{r-m}} \kappa-i K^{r-m-1-q} T}^{N_{n_{r-m}} \kappa+i K^{r-m-1-q} T} \frac{1}{\zeta\left(1+s_{n_{r-m}}\right) s_{n_{r-m}}} \\
& \times \prod_{i=r-m+1}^{r} \eta^{\left(a_{i, m}\right)}\left(T_{(m)} s_{n_{i}}\right) \prod_{v \in \mathcal{U}_{P} \backslash V_{m}} \zeta\left(P, m, v, i_{n_{2}}, \ldots, i_{n_{m}}, s\right) \\
& \times \prod_{v \in \mathcal{U}_{I}^{*} \backslash V_{m}} \zeta\left(I, m, v, j_{n_{2}}, \ldots, j_{n_{m}}, s\right) H_{r-m}(s) x^{T_{(m)} v_{0} s} d s_{n_{r-m}} \cdots d s_{n_{1}},
\end{aligned}
$$

où $P_{m}$ est un polynôme, où $s_{n_{i}}(i=1, \ldots, r)$ est une permutation (dépendant du terme considéré, et bien sûr de $m)$ des variables $s_{j}(j=1, \ldots, r)$ où $K>2$ est la constante de (63) et $q$ le nombre d'applications du procédé (b) du Paragraphe 2.3.2 jusqu'ici, où les applications linéaires $T_{i}$ satisfont les hypothèses du Paragraphe 2.4 (sur les variables $\bar{s}_{i}=s_{n_{i}}$ ), où l'on note abusivement $T_{(m)} s_{n_{i}}$ pour $T_{(m)}\left[\begin{array}{c}0 \\ \vdots \\ 0 \\ s_{n_{i}} \\ 0 \\ \vdots \\ 0\end{array}\right]\left(s_{n_{i}}\right.$ étant le $i$-ème coefficient du vecteur), où $H_{r-m}(s)$ est une fonction des variables $s_{n_{i}}(i=1, \ldots, r-m)$, régulière et bornée pour $\left|\sigma_{n_{i}}\right| \geq c$, avec $c=c(r)>0$, et où par le Lemme 11,

$$
\eta^{\left(a_{i, m}\right)}\left(T_{(m)} s_{n_{i}}\right)=\frac{A_{N}\left(T_{(m)} s_{n_{i}}\right)}{s+1} \quad \text { avec }\left|A_{N}(s)\right| \leq \varepsilon(x)^{-1} .
$$

Remarque 12. Notons que, dans le cas où l'exposant de $x$ est identiquement nul et où nous cessons donc d'appliquer les procédés (a) et (b) décrits dans le Paragraphe 2.3.2, les termes obtenus sont également du type (18) (avec évidemment $T_{(m)} v_{0}=\mathcal{O}$ ).

(a) Soit $b>0$ une constante qui sera précisée un peu plus loin (voir le Lemme 17). Dans le cas où le coefficient $c=c_{n_{r-m}}$ de $s_{n_{r-m}}$ dans l'exposant 
$T_{(m)} v_{0} s$ n'est pas nul (c'est-à-dire, si nous sommes en position d'appliquer le procédé (a) du Paragraphe 2.3.2), nous déformons le segment vertical d'intégration de $N_{n_{r-m}} \kappa-i K^{r-m-1-q} T$ à $N_{n_{r-m}} \kappa+i K^{r-m-1-q} T$, que nous appelons $I_{m}$, en parcourant les segments horizontaux (dans le sens adéquat) de $N_{n_{r-m}} \kappa \pm i K^{r-m-1-q} T$ à $\operatorname{sgn}(-c) b \beta \pm i K^{r-m-1-q} T$, que nous appelons $I_{m, \pm}$, et le segment vertical de $\operatorname{sgn}(-c) b \beta-i K^{r-m-1-q} T$ à $\operatorname{sgn}(-c) b \beta+$ $i K^{r-m-1-q} T$, que nous appelons $J_{m, b}$. Soit aussi $F_{m}:=I_{m} \cup I_{m,+} \cup I_{m,-} \cup J_{m, b}$ la frontière du rectangle. Si $R_{m}$ désigne la somme des résidus de l'intégrand à l'intérieur de $F_{m}$, le terme en (18) peut donc s'écrire

$$
\begin{aligned}
& P_{m}(\log x) \int_{N_{n_{1}} \kappa-i K^{-q} T}^{N_{n_{1}} \kappa+i K^{-q} T} \frac{1}{\zeta\left(1+s_{n_{1}}\right) s_{n_{1}}} \cdots \\
& \quad \ldots\left(\left(\left(\int_{I_{m,-}}+\int_{J_{m, b}}+\int\right) \frac{1}{\zeta\left(1+s_{n_{r-m}}\right) s_{n_{r-m}}} \prod_{i=r-m+1}^{r} \eta^{\left(a_{i, m}\right)}\left(T_{(m)} s_{n_{i}}\right)\right.\right. \\
& \quad \times \prod_{v \in \mathcal{U}_{P} \backslash V_{m}} \zeta\left(P, m, v, i_{n_{2}}, \ldots, i_{n_{m}}, s\right) \prod_{v \in \mathcal{U}_{I}^{*} \backslash V_{m}} \zeta\left(I, m, v, j_{n_{2}}, \ldots, j_{n_{m}}, s\right) \\
& \left.\left.\times H_{r-m}(s) x^{T_{(m)} v_{0} s} d s_{n_{r-m}}\right)+R_{m}\right) d s_{n_{r-m-1}} \cdots d s_{n_{1}} \\
& =: \mathcal{I}_{m,-}+\mathcal{J}_{m, b}+\mathcal{I}_{m,+}+\mathcal{R}_{m},
\end{aligned}
$$

où, par le Lemme 8 et le Paragraphe 2.7 (voir l'expression (51)), $\mathcal{R}_{m}$ est une somme de termes de la forme

$$
\begin{aligned}
& P_{m+1}(\log x) \int_{N_{n_{1}} \kappa-i K^{-q} T}^{N_{n_{1}} \kappa+i K^{-q} T} \frac{1}{\zeta\left(1+s_{n_{1}}\right) s_{n_{1}}} \cdots \\
& \quad \ldots \quad \int^{N_{n_{r-m-1}}^{\kappa+i K^{r-m-2-q} T}} \frac{1}{\zeta\left(1+s_{n_{r-m-1}}\right) s_{n_{r-m-1}}} \int^{N_{n_{r-m-1}} \kappa-i K^{r-m-2-q} T} \\
& \quad \times \prod_{i=r-m}^{r} \eta^{\left(a_{i, m+1}\right)}\left(T_{(m+1)} s_{n_{i}}\right) \prod_{v \in \mathcal{U}_{P} \backslash V_{m+1}} \zeta\left(P, m+1, v, i_{n_{2}}, \ldots, i_{n_{m+1}}, s\right) \\
& \quad \times \prod_{v \in \mathcal{U}_{I}^{*} \backslash V_{m+1}} \zeta\left(I, m+1, v, j_{n_{2}}, \ldots, j_{n_{m+1}}, s\right) \\
& \quad \times H_{r-m-1}(s) x^{T_{(m+1)} v_{0} s} d s_{n_{r-m-1}} \cdots d s_{n_{1}},
\end{aligned}
$$

c'est-à-dire de la forme (18) avec $m+1$ à la place de $m$. Nous allons montrer que $\mathcal{I}_{m,-}+\mathcal{J}_{m, b}+\mathcal{I}_{m,+} \ll \delta(x)$. 
Jusqu'à l'énoncé du Lemme 17 ci-dessous nous simplifions à nouveau un peu la notation et rebaptisons $s_{j}$ la variable $s_{n_{j}}$ (cf. la Remarque 6). Nous avons besoin d'estimations, lorsque la variable $s_{r-m}$ se trouve sur le chemin $P_{n}$, pour les fonctions, qui ont été introduites aux Paragraphes 2.6 (pour $0 \leq m \leq 3)$ et 2.7 , de types $\zeta\left(P, m, v, i_{2}, \ldots, i_{m}, s\right)$ et $\zeta\left(I, m, v, j_{2}, \ldots, j_{m}, s\right)$.

Rappelons que $\zeta(P, 0, v, s)=\zeta(1+v s)$ où $v \in \mathcal{U}_{P}$, que $\zeta(P, 1, v, s)=$ $\zeta\left(1+c_{1} s_{1}+\cdots+c_{r} s_{r}\right)=: g_{1}(s)$ où les coefficients $c_{i}$ sont réels (en fait, ils sont $0,+1$, ou -1$)$, et que, pour $m \geq 2, \zeta\left(P, m, v, i_{2}, \ldots, i_{m}, s\right)$ est obtenue à partir de $g_{1}(s)$ en lui faisant subir une succession d'opérations. Cette fonction est d'abord dérivée $i_{2} \geq 0$ fois par rapport à une des variables $s_{i}$, et dans le résultat obtenu $s_{i}$ est remplacé par une combinaison linéaire des autres variables, livrant ce que nous pouvons noter $g_{2}(s)$. La fonction $g_{2}(s)$ est alors à son tour dérivée $i_{3} \geq 0$ fois par rapport à une autre variable $s_{j}$, et dans le résultat obtenu $s_{j}$ est remplacé par une combinaison linéaire des variables restantes. Finalement, $\zeta\left(P, m, v, i_{2}, \ldots, i_{m}, s\right)$ est la fonction obtenue en dérivant $g_{m-1}(s) i_{m}$ fois par rapport à une des variables restantes $s_{k}$, puis en remplaçant dans le résultat $s_{k}$ par une combinaison linéaire des autres variables restantes. Par conséquent, on peut écrire

$$
\zeta\left(P, m, v, i_{2}, \ldots, i_{m}, s\right)=C \zeta^{\left(i_{0}\right)}\left(1+\gamma\left(s_{1}, \ldots, s_{r}\right)\right),
$$

où $C$ est une constante réelle, $\gamma$ une combinaison linéaire non identiquement nulle des variables $s_{1}, \ldots, s_{r}$, et $i_{0}=i_{2}+\cdots+i_{m}$.

De même $\zeta(I, 0, v, s)=\zeta(1+v s)^{-1}$ où $v \in \mathcal{U}_{I}^{*}, \zeta(I, 1, v, s)=1 / \zeta(1+$ $\left.c_{1} s_{1}+\cdots+c_{r} s_{r}\right)=: h_{1}(s)$ où les coefficients $c_{i}$ sont réels et, pour $m \geq 2$, $\zeta\left(I, m, v, j_{2}, \ldots, j_{m}, s\right)$ est obtenue à partir de $h_{1}(s)$ en lui faisant subir une succession d'opérations similaires. On a donc aussi l'écriture

$$
\zeta\left(I, m, v, j_{2}, \ldots, j_{m}, s\right)=C^{\prime}(1 / \zeta)^{\left(j_{0}\right)}\left(1+\gamma^{\prime}\left(s_{1}, \ldots, s_{r}\right)\right),
$$

où $C^{\prime}$ est une constante réelle, $\gamma^{\prime}$ une combinaison linéaire non identiquement nulle des variables $s_{1}, \ldots, s_{r}$, et $j_{0}=j_{2}+\cdots+j_{m}$.

Le lemme ci-dessous suit maintenant des identités (67) et (68), du Lemme 10, de l'estimation (25), et du Lemme 16.

LEMme 17. Il existe une constante positive $b$ absolue (qu'on pourra supposer inférieure aux constantes $A$ et a des Lemmes 11 et 12) telle que

$$
\prod_{v \in \mathcal{U}_{P} \backslash V_{m}} \zeta\left(P, m, v, i_{n_{2}}, \ldots, i_{n_{m}}, s\right) \prod_{v \in \mathcal{U}_{I}^{*} \backslash V_{m}} \zeta\left(I, m, v, j_{n_{2}}, \ldots, j_{n_{m}}, s\right) \leq \varepsilon(x)^{-1}
$$

pour tout $m=0,1, \ldots, l$ et tous les termes de type (18) intervenant dans l'évaluation de $I$, pour autant que pour $s_{j}=\sigma_{j}+i t_{j}(j=1, \ldots, r)$, on ait $\left\{\left|\sigma_{j}\right| \leq b /(\log T)^{2 / 3}(\log \log T)^{1 / 3}\right\}$, que pour une constante positive $C$, $\left|t_{j}\right| \leq C T(j=1, \ldots, r)$, et que $s_{n_{r-m}} \in F_{m}$. 
Lemme 18. Avec la notation de (66) on a $\left|\mathcal{I}_{m,-}+\mathcal{J}_{m, b}+\mathcal{I}_{m,+}\right| \ll \delta(x)$.

Preuve. Sur les chemins d'intégration considérés on a en effet $H_{r-m}(s)$ $\ll 1$ par le Lemme $9, x^{T_{(m)} v_{0} s} \ll x^{-|c| \beta}=\delta(x)$ par définition de $J_{m, b}$ et des paramètres $\kappa$ et $\beta$,

$$
\prod_{v \in \mathcal{U}_{P} \backslash V_{m}} \zeta\left(P, m, v, i_{n_{2}}, \ldots, i_{n_{m}}, s\right) \prod_{v \in \mathcal{U}_{I}^{*} \backslash V_{m}} \zeta\left(I, m, v, j_{n_{2}}, \ldots, j_{n_{m}}, s\right) \leq \varepsilon(x)^{-1}
$$

par le Lemme 17,

$$
\prod_{i=r-m+1}^{r} \eta^{\left(a_{i, m}\right)}\left(T_{(m)} s_{n_{i}}\right) \leq \varepsilon(x)^{-1}
$$

par le Lemme 11, et

$$
\frac{1}{\zeta\left(1+s_{n_{j}}\right) s_{n_{j}}} \ll \frac{(\log x)^{2 / 5}(\log \log x)^{1 / 5}}{\left|t_{n_{j}}\right|+1} \quad(j=1, \ldots, r-m)
$$

par (25) et la définition de $T$.

Par conséquent, nous avons (voir (23))

$$
\begin{aligned}
\mathcal{J}_{m, b} & \ll \delta(x) \int_{-K^{-q} T}^{+K^{-q} T} \frac{1}{\left|t_{n_{1}}\right|+1} \cdots \int_{-K^{r-m-1-q} T}^{+K^{r-m-1-q} T} \frac{1}{\left|t_{n_{r-m}}\right|+1} d t_{n_{r-m}} \cdots d t_{n_{1}} \\
& \ll \delta(x)
\end{aligned}
$$

et

$$
\begin{aligned}
\mathcal{I}_{m, \pm} & \ll \delta(x) \int_{-K^{-q} T}^{+K^{-q} T} \frac{1}{\left|t_{n_{1}}\right|+1} \cdots \\
& \quad \ldots \quad \int_{-K^{r-m-2-q} T} \frac{1}{\left|t_{n_{r-m-1}}\right|+1} \int \frac{1}{T} d \sigma_{n_{r-m}} d t_{n_{r-m-1}} \cdots d t_{n_{1}} \\
& \ll \delta(x),
\end{aligned}
$$

où le chemin d'intégration de l'intégrale intérieure a pour bornes les nombres $\operatorname{sgn}(-c) b \beta$ et $N_{n_{r-m}} \kappa$.

(b) Dans le cas où le coefficient $c=c_{n_{r-m}}$ de $s_{n_{r-m}}$ dans l'exposant $T_{(m)} v_{0} s$ est nul, mais où au moins un des coefficients des autres variables n'est pas nul (c'est-à-dire, si nous sommes en position d'appliquer le procédé (b) du Paragraphe 2.3.2), alors dans (15) nous commençons par diviser la longueur $2 K^{r-m-1-q} T$ du segment d'intégration par rapport à la variable $s_{n_{r-m}}$ par $K^{r-m}$, puis nous permutons les variables et intégrons par rapport à $s_{n_{r-m-1}}, \ldots, s_{n_{1}}, s_{n_{r-m}}$ dans l'ordre indiqué, que nous rebaptisons $s_{\nu_{r-m}}, \ldots, s_{\nu_{2}}, s_{\nu_{1}}$. Nous obtenons ainsi une expression du type (18), où l'indice $n_{j}$ est remplacé par $\nu_{j}(j=1, \ldots, r-m)$, et $q$ par $q+1$. 
LEMME 19. Après la modification du chemin d'intégration par rapport à la variable $s_{n_{r-m}}$ décrite ci-dessus, le terme obtenu diffère du terme en (18) d'une quantité qui, en valeur absolue, est $\ll \delta(x)$.

Preuve. Si $c=c_{n_{r-m}}=0$, alors au moins un $\operatorname{des} T_{(m)} s_{n_{j}}=\sum_{i \leq r-m} d_{j, i} s_{n_{i}}$ $(j \geq r-m+1)$ a un coefficient $d_{j, r-m}=D \neq 0$, puisque $c$ est la somme des coefficients en $s_{n_{r-m}}$ de tous les $T_{(m)} s_{n_{j}}(j \geq r-m)$, et puisque $T_{(m)} s_{n_{r-m}}$ $=s_{n_{r-m}}$. Nous vérifions que, si $Q$ est une constante positive,

$$
\int_{0}^{Q T} \frac{d t_{n_{r-m}}}{\left|T_{(m)}\left(t_{n_{j}}\right)\right|+1} \leq \frac{2}{D} \log \left(1+|D| Q T+\left|\sum_{i \neq r-m} d_{j, i} t_{n_{i}}\right|\right) \leq \varepsilon(x)^{-1} .
$$

D'autre part, les majorations (69) et (70) restent valables, et l'on a bien sûr $x^{T_{(m)} v_{0} s}=x^{O(\kappa)} \ll 1$. Par conséquent, il suit du Lemme 12 que la différence que nous voulons estimer est

$$
\begin{aligned}
\leq & \varepsilon(x)^{-1} \int_{-K^{-q} T}^{+K^{-q} T} \frac{1}{\left|t_{n_{1}}\right|+1} \cdots \int_{-K^{r-m-2-q} T}^{+K^{r-m-2-q} T} \frac{1}{\left|t_{n_{r-m-1}}\right|+1} \\
& \times \int^{+K^{r-m-1-q} T} \frac{1}{\mid K^{-1-q} T} \frac{1}{\left|t_{n_{r-m}}\right|+1} \frac{1}{\left|T_{(m)}\left(t_{n_{j}}\right)\right|+1} d t_{n_{r-m}} \cdots d t_{n_{1}} \\
\leq & \frac{\varepsilon(x)^{-1}}{T} \int_{-K^{-q} T}^{+K^{-q} T} \frac{1}{\left|t_{n_{1}}\right|+1} \cdots \\
& \quad \ldots \quad \int^{r-m-2-q} T \\
\leq & \frac{\varepsilon(x)^{-1}}{T} \ll \delta(x) .
\end{aligned}
$$

Nous considérons maintenant chacune des situations finales possibles (f1) et (f2) décrites au Paragraphe 2.3.2, afin de terminer la démonstration du Théorème 1.

CAS (f1). Après l'ultime l-ème étape de l'évaluation, pour la suite de choix de pôles $s_{n_{r}}^{*}, \ldots, s_{n_{r-l+1}}^{*}$, l'exposant $T_{(l)} v_{0} s$ de $x^{T_{(l)} v_{0} s}$ n'est pas identiquement nul, alors que le résidu final obtenu, qui doit encore être intégré au moins une fois par le Lemme 14, n'a plus aucune singularité. On peut donc supposer que le coefficient $c_{n_{r-l}}$ de $s_{n_{r-l}}$ dans $T_{(l)} v_{0} s$ n'est pas nul. Il suit, par le Lemme 18, que la contribution d'un tel terme à $I$ est $\ll \delta(x)$. En effet, le terme qu'il nous reste à évaluer est de la forme (18), et plus précisément (66), avec $l$ à la place de $m, c=c_{n_{r-l}} \neq 0$ et $\mathcal{R}_{l}=0$.

Comme nous l'avons déjà mentionné, ceci termine, avec le Lemme 15, la démonstration du (ii) du Théorème 1. 
CAS (f2). Après l'ultime l-ème étape de l'évaluation, pour la suite de choix de pôles $s_{n_{r}}^{*}, \ldots, s_{n_{r-l+1}}^{*}$ l'exposant $T_{(l)} v_{0} s$ de $x^{T_{(l)} v_{0} s}$ est identiquement nul, alors que le résidu final obtenu doit encore être intégré au moins une fois, toujours par le Lemme 14. La contribution de ce résidu peut s'écrire sous la forme (18), avec $l$ à la place de $m$ et $T_{(l)} v_{0} s=0$. Il peut encore éventuellement avoir des pôles (mais évidemment pas sur les chemins d'intégration de (18)). La preuve du lemme qui suit termine donc la démonstration du (i) du Théorème 1.

Lemme 20. Notons dorénavant

$$
\kappa=\kappa(x)=1 / \log x \quad \text { et } \quad T=T(x) .
$$

Posons

$$
\tau(x):=K^{-q} T(x)
$$

L'intégrale $r$ - l-uple

$$
\begin{aligned}
a= & a(x) \\
= & \int_{N_{n_{1}} \kappa(x)-i \tau(x)}^{N_{n_{1}} \kappa(x)+i \tau(x)} \frac{1}{\zeta\left(1+s_{n_{1}}\right) s_{n_{1}}} \cdots \int_{N_{n_{r-l}} \kappa(x)-i K^{r-l-1} \tau(x)}^{N_{n_{r-l}} \kappa(x)+i K^{r-l-1} \tau(x)} \frac{1}{\zeta\left(1+s_{n_{r-l}}\right) s_{n_{r-l}}} \\
& \times \prod_{i=r-l+1}^{r} \eta^{\left(a_{i, l}\right)}\left(T_{(l)} s_{n_{i}}\right) \prod_{v \in \mathcal{U}_{P} \backslash V_{l}} \zeta\left(P, l, v, i_{n_{2}}, \ldots, i_{n_{l}}, s\right) \\
& \times \prod_{v \in \mathcal{U}_{I}^{*} \backslash V_{l}} \zeta\left(I, l, v, j_{n_{2}}, \ldots, j_{n_{l}}, s\right) H_{r-l}(s) d s_{n_{r-l}} \cdots d s_{n_{1}}
\end{aligned}
$$

peut s'écrire sous la forme $C+O(\delta(x))$, où $C$ est une constante.

Preuve. Si l'intégrand de (72) ne contient plus de pôle, le résultat peut se montrer en déplaçant successivement toutes les abscisses d'intégration sur l'abscisse 0. Mais si l'intégrand contient encore des pôles, les déplacements d'abscisses ne peuvent pas se faire jusqu'à l'abscisse 0 et ne peuvent être que très restreints. Nous procédons de façon similaire à la preuve du Lemme 16 . Un pôle $\left(\bar{s}_{n_{1}}, \ldots, \bar{s}_{n_{r-l}}\right)$ de l'intégrand correspond à une expression linéaire $\gamma\left(s_{n_{1}}, \ldots, s_{n_{r-l}}\right)=c_{1} s_{n_{1}}+\cdots+c_{r-l} s_{n_{r-l}}$, apparaissant dans l'argument d'un ou plusieurs facteurs de l'intégrand (voir (67) et (68)) et telle que $\gamma\left(\bar{s}_{n_{1}}, \ldots, \bar{s}_{n_{r-l}}\right)=0$. En suivant de près l'argument de la preuve du Lemme 16 , on montre que, pour $x$ assez grand, si $\sigma_{n_{j}} \in\left[N_{n_{j}} \kappa(2 x), N_{n_{j}} \kappa(x)\right](j=$ $1, \ldots, r-l)$ alors

$$
\gamma\left(s_{n_{1}}, \ldots, s_{n_{r-l}}\right) \geq \frac{C_{-}}{2} \kappa(x) .
$$


Nous faisons maintenant appel à l'intégrale $r-l$-uple (l'intégrand étant le même qu'en (72))

$$
b=b(x)=\int_{N_{n_{1}} \kappa(2 x)-i \tau(x)}^{N_{n_{1}} \kappa(2 x)+i \tau(x)} \cdots \int_{N_{n_{r-l}} \kappa(2 x)-i K^{r-l-1} \tau(x)}^{N_{n_{r-l}} \kappa(2 x)+i K^{r-l-1} \tau(x)} \cdots d s_{n_{r-l}} \cdots d s_{n_{1}} .
$$

Notons, pour un $x_{0}>0$ assez grand, $x_{M}:=2 x_{M-1}, a_{M}:=a\left(x_{M}\right)$ et $b_{M}:=b\left(x_{M}\right)(M \geq 1)$. Pour démontrer le lemme nous établissons la convergence de la suite $\left\{a_{M}\right\}$, en montrant qu'elle est une suite de Cauchy. Afin d'évaluer $a_{M+1}-a_{M}$, nous écrivons $a_{M+1}-a_{M}=\left(a_{M+1}-b_{M}\right)+\left(b_{M}-a_{M}\right)$, et nous commençons par évaluer $b_{M}-a_{M}$. Pour ce faire, nous remplaçons successivement chacun des $r-l$ chemins d'intégration de $a_{M}$ par le chemin correspondant de $b_{M}$. Chaque erreur ainsi commise est estimée, avec l'aide de (73) (qui nous garantit de plus l'absence de pôle dans la région du plan balayée par la déformation du contour d'intégration), en reproduisant l'argument du Lemme 18 pour l'estimation de $\mathcal{I}_{m, \pm}$ (et en exploitant cette fois le facteur $1 / T=\delta(x))$. Nous montrons ainsi que $\left|b_{M}-a_{M}\right| \ll \delta(x)$.

Puis nous évaluons $a_{M+1}-b_{M}$. Pour ce faire, nous allongeons successivement chacune des $r-l$ abscisses d'intégration de $b_{M}$, dont la longueur est multipliée par $T(2 x) / T(x)$. Cette fois nous répétons $r-l$ fois un argument similaire à la preuve du Lemme 19, en utilisant la propriété suivante, qui garantit que (71) est applicable : puisque $T_{(l)} v_{0} s=s_{n_{1}}+\cdots+s_{n_{r-l}}+$ $T_{(l)} s_{n_{r-l+1}}+\cdots+T_{(l)} s_{n_{r}}$ est identiquement nul, pour chaque $h=1, \ldots, r-l$ un au moins des $T_{(l)} s_{n_{j}}(j \geq r-l+1)$ a un coefficient non nul en $s_{n_{h}}$. Nous montrons ainsi que $\left|a_{M+1}-b_{M}\right| \ll \delta(x)$. tion.

La vérification de l'estimation $\sum_{i \geq 0} \delta\left(2^{i} x\right) \ll \delta(x)$ termine la démonstra-

\section{Références}

[B] R. de la Bretèche, Estimation de sommes multiples de fonctions arithmétiques, Compos. Math. 128 (2001), 261-298.

[D] H. Delange, Sur les fonctions de plusieurs entiers strictement positifs, Enseign. Math. 15 (1969), 77-88.

[DIT] F. Dress, H. Iwaniec et G. Tenenbaum, Sur une somme liée à la fonction de Möbius, J. Reine Angew. Math. 340 (1983), 53-58.

[M1] Y. Motohashi, Möbius function over divisors, manuscrit non publié daté du 15 mars 1985.

[M2] - - A multiple sum involving the Möbius function, Publ. Inst. Math. (Beograd) (N.S.) 76 (90) (2004), 31-39.

[R] S. Ramanujan, Some formula in the analytic theory of numbers, Messenger Math. 45 (1916), 81-84; or Collected Papers, Cambridge Univ. Press, 1927, 133-135. 
[T] G. Tenenbaum, Introduction à la théorie analytique et probabiliste des nombres, Soc. Math. France, Paris, 1995.

[THB] E. C. Titchmarsh, The Theory of the Riemann Zeta-Function, Clarendon Press, Oxford, 1951; 2nd ed. revised by D. R. Heath-Brown, 1986.

[W] B. M. Wilson, Proofs of some formulce enunciated by Ramanujan, Proc. London Math. Soc. 21 (1923), 235-255.

Institut de Mathématiques de Luminy

Université de la Méditerranée Aix-Marseille 2

Campus de Luminy Case 907

13288 Marseille Cedex 9, France

E-mail: balazard@iml.univ-mrs.fr

Section de Mathématiques

Université de Genève

2-4 rue du Lièvre, CP 64

1211 Genève 4, Suisse

E-mail: Petermann@math.unige.ch
Département de Mathématiques Faculté des Sciences de Tunis 1060 Tunis, Tunisie

E-mail: Mongi.Naimi@fst.rnu.tn

Reçu le 23.1.2007

et révisé le 25.1.2008 